\title{
THESIS
}

\section{SUCCESSION OF THE IMAGE}

\author{
by \\ Matthew Koniuszewski, \\ B. Arch Sci, \\ Ryerson University, 2013 \\ A design thesis \\ Presented to Ryerson University \\ In partial fulfillment of the \\ requirements for the degree of \\ Master of Architecture \\ in the Program of \\ Architecture
}

Toronto, Ontario, Canada, 2016

(CMatthew Koniuszewski 2016 



\section{AUTHOR'S DECLARATION}

I hereby declare that I am the sole author of this thesis. This is a true copy of the thesis, including any required final revisions, as accepted by my examiners.

I authorize Ryerson University to lend this thesis to other institutions or individuals for the purpose of scholarly research.

Matthew Koniuszewski

I further authorize Ryerson University to reproduce this thesis by photocopying or by other means, in total or in part, at the request of other institutions or individuals for the purpose of scholarly research.

I understand that my thesis may be made electronically available to the public.

Matthew Koniuszewski 



\section{ABSTRACT \\ SUCCESSION OF THE IMAGE \\ Matthew Koniuszewski, M.Arch 2016 \\ Master of Architecture \\ Ryerson University}

As technology evolves and spheres of information dissemination begin to converge, now is a crucial point to become critical with how these factors are beginning to influence architecture. It is important to understand the ways in which we consume architecture is affecting the architecture we produce. Is architecture a handful of money-shot renderings creating idealised moments captioned with generic copy and paste, or buried in books on shelves? Or have we as architects decided that mechanical reproduction is unable to capture the essence of architecture and that it can only be experienced?

The succession of the image is the challenging of conventional methods of communication utilized by the architectural artifact. In focusing on the possibilities afforded via technological advances emerges a variety of mediums for the creation of spatial narratives. The image has progressed and disseminated architecture to this point and it is important to continuously ask what is next. 



\section{ACKNOWLEDGMENTS}

I want to thank my partner for all her love and support, my family and friends who stood by me during this trying year and my advisors, Kendra Schank Smith and second reader Marco Polo for pushing me and believing in me. 



\section{TABLE OF CONTENTS}

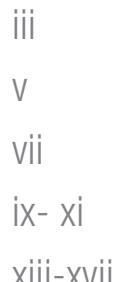

Xiii-Xvii

01

05

09

13

17

21

25

25

28

35

35

36

37

38

39

\author{
AUTHOR'S DECLARATION \\ ABSTRACT \\ ACKNOWLEDGMENTS \\ TABLE OF CONTENTS \\ LIST OF FIGURES
}

INTRODUCTION: MISE-EN-SCENE

METHODS OF COMMUNICATION

THE BEAUTIFUL UNCERTAINTY OF PIECES

THE SPACE BETWEEN THE STROKES

REMIX AND THE 21ST CENTURY ARTIFACT

SEQUENCE AND ILLUSION

DECONSTRUCTING ARTIFACT CONSTRUCTION

LAYERING

TRIPARTE DIAGRAM

PRECEDENTS

SPIRAL

PERIMETER

CLOUD

EXPOSURE

NARRATIVE 
41

41

42

43

45

51

53

53

56

59

61

65

65

72

74

78

81

81

83

85

CONCEPTUAL MAPS

DISTANCING

DISORIENTATION

EXPOSURE

PROGRAMMATIC EXPOSURE

SITE ANALYSIS

SHADOW BOX

SHADOW BOX

REALIZING THE SHADOW BOX

CHANGING LOCATION

SECTIONAL MODEL

DESIGN 1: CIRCULATORY NARRATIVE

MASSING

PARTIAL MODEL

VIGNETTE

UNFOLDED CIRCULATORY NARRATIVE EXPERIMENT

DESIGN 2: VERTICAL NARRATIVE

RE-ANALYZING DUNDAS SQUARE

FIRST DESIGN EXPERIMENT

SECOND DESIGN EXPERIMENT

91

GONCLUSIOA 
APPENDIX

I. DESIGN ITERATION 1 DRAWINGS

101

II. SOUND STAGE / STUDIO PRECEDENTS

BIBLIOGRAPHY 



\section{LIST OF FIGURES}

\begin{tabular}{|c|c|c|}
\hline PAGE 05 & FIGURE 1 & $\begin{array}{l}\text { DIAGRAM } \\
\text { Thesis Structure: Filtered }\end{array}$ \\
\hline 06 & FIGURE 2 & $\begin{array}{l}\text { DIAGRAM } \\
\text { Thesis Structure: Multi-Faceted }\end{array}$ \\
\hline 06 & FIGURE 3 & $\begin{array}{l}\text { DIAGRAM } \\
\text { Thesis Structure: Multi-Faceted Unfolded }\end{array}$ \\
\hline 10 & FIGURE 4 & $\begin{array}{l}\text { DIAGRAM } \\
\text { 21st Century Museum of Contemporary Art - SANAA - Fragment to } \\
\text { boundary analysis }\end{array}$ \\
\hline
\end{tabular}

11 FIGURE 5 DIAGRAM

21st Century Museum of Contemporary Art - SANAA - Fragment to boundary analysis

12 FIGURE 6 PAINTING

Fighting Temeraire - Turner

Source: http://uploads3. wikiart.org/images/william-turner/the-fightingtemeraire-tugged-to-her-last-berth-to-be-broken-up.jpg

12 FIGURE $7 \quad$ FILM

Still from "Tom Keating on Painters - Keating"

Source: https://www.youtube.com/watch?v=MDmiOmYnwKk (24:04)

14 FIGURE 8 DIAGRAM

Columbia College Chicago Media Production Centre - Studio Gang -

Circulation Diagram

Source: http://studiogang. net/sites/default/files/styles/large/public/

cccmpc6.jpg

15 FIGURE 9 FILM

Still from CBC News Vancouver @ 6

Source: http://www.cbc.ca/player/News/Canada/BC/

ID/2652228271/ (16:31)

19 FIGURE 10 DIAGRAM

UN Studio Design Model

Source: http://www.archined.nl/uploads/media/Design_models_ p22-p23.jpg

21 FIGURE $11 \quad$ FILM

Film still from Memento (2000)

Source: http://www.archined.nl/uploads/media/Design_models_ p22-p23.jpg

22 FIGURE 12 MODEL

Section Based Experimental Model 
PRECEDENT

$\mathrm{A}+\mathrm{B}=\mathrm{C}$ Barbara Kruger (1978)

Source: http://artistsbooks.info/AB_Kruger\%20Barbara_Picture\%20

readings.html

26 FIGURE 14 EXPERIMENT

Illustrative Captioning

27 FIGURE $15 \quad$ EXPERIMENT

Text Based Atmospheric Rendering

Source: http://gradhouse.utoronto.ca/staff/

29 FIGURE 16 EXPERIMENT

Triparte Diagram: Constructing Advertising

30 FIGURE 17 EXPERIMENT

Triparte Diagram: Fold

31 FIGURE 18 EXPERIMENT

Triparte Diagram: Slice

32 FIGURE 19 EXPERIMENT

Triparte Diagram: Atomize

33 FIGURE 20 EXPERIMENT

Triparte Diagram: Distance

34 FIGURE 21 EXPERIMENT

Triparte Diagram: Disorient

35 FIGURE 22 PRECEDENT

\section{NEW MEDIA CAMPUS COMPETITION}

Bjarke Ingels Group.Berlin, Germany

Source: http://www.archdaily.com/310435/tv-azteca-film-tv-sets-

campus-at103

36 FIGURE 23 PRECEDENT

TV AZTECA FILM \& TV SETS CAMPUS 2012

at103. Tezozomoc, Xico, Mexico

Source: http://www.archdaily.com/310435/tv-azteca-film-tv-sets-

campus-at103

36 FIGURE 24 PRECEDENT

MEDIA LIBRARY AND CULTURAL CENTRE 2010

Barbotin + Gresham Architects. Lisses, France

Source: http://www.archdaily.com/office/barbotin-gresham-

architects

$37 \quad$ FIGURE $25 \quad$ PRECEDENT

NEW MEDIA CAMPUS COMPETITION

OMA. Berlin, Germany.

Source: http://www.archdaily.com/459281/big-oma-buro-os-to-

compete-for-new-media-campus-in-berlin 
PAGE 37 FIGURE 26 PRECEDENT

NEW MEDIA CAMPUS COMPETITION

Buro-OS. Berlin, Germany.

Source: http://www. archdaily.com/459281/big-oma-buro-os-to-

compete-for-new-media-campus-in-berlin

38 FIGURE 28 PRECEDENT

NMIT ARTS \& MEDIA 2010

Irving Smith Jack Architects. Nelson, New Zealand.

Source: http://openbuildings.com/buildings/new-zealands-landmark-

timber-structural-demonstration-building-nmit-arts-and-media-building-

profile-41550

$38 \quad$ FIGURE $27 \quad$ PRECEDENT

CBC BROADCASTING CENTRE EXPANSION 2010

DIALOG Design. Vancouver.

Source: http://www.cbc.radio-canada.ca/en/explore/who-we-are-what-

we-do/canadian/

39 FIGURE 29 PRECEDENT

\section{COLUMBIA COLLEGE CHICAGO MEDIA}

PRODUCTION CENTER 2010

Studio Gang Architects. Chicago, USA

Source: http://studiogang.net/sites/default/files/styles/large/public/

ccompc6.jpg

40 FIGURE 30 DIAGRAM

Conceptual Map: Distance

42 FIGURE 31 DIAGRAM

Conceptual Map: Disorientate

43 FIGURE 32 DIAGRAM

Conceptual Map: Exposure

44 FIGURE 33 DIAGRAM

Programming Analysis: Raw to Final Product

45 FIGURE 34 LEGEND

Programing and Scale

46 FIGURE 35 DIAGRAM

Programming Analysis: Spacial Relationship

47 FIGURE 36 DIAGRAM

Programming Relationship: Interdisciplinary Clustering

48 FIGURE 37 DIAGRAM

Programming Analysis, Sectional Relationship 


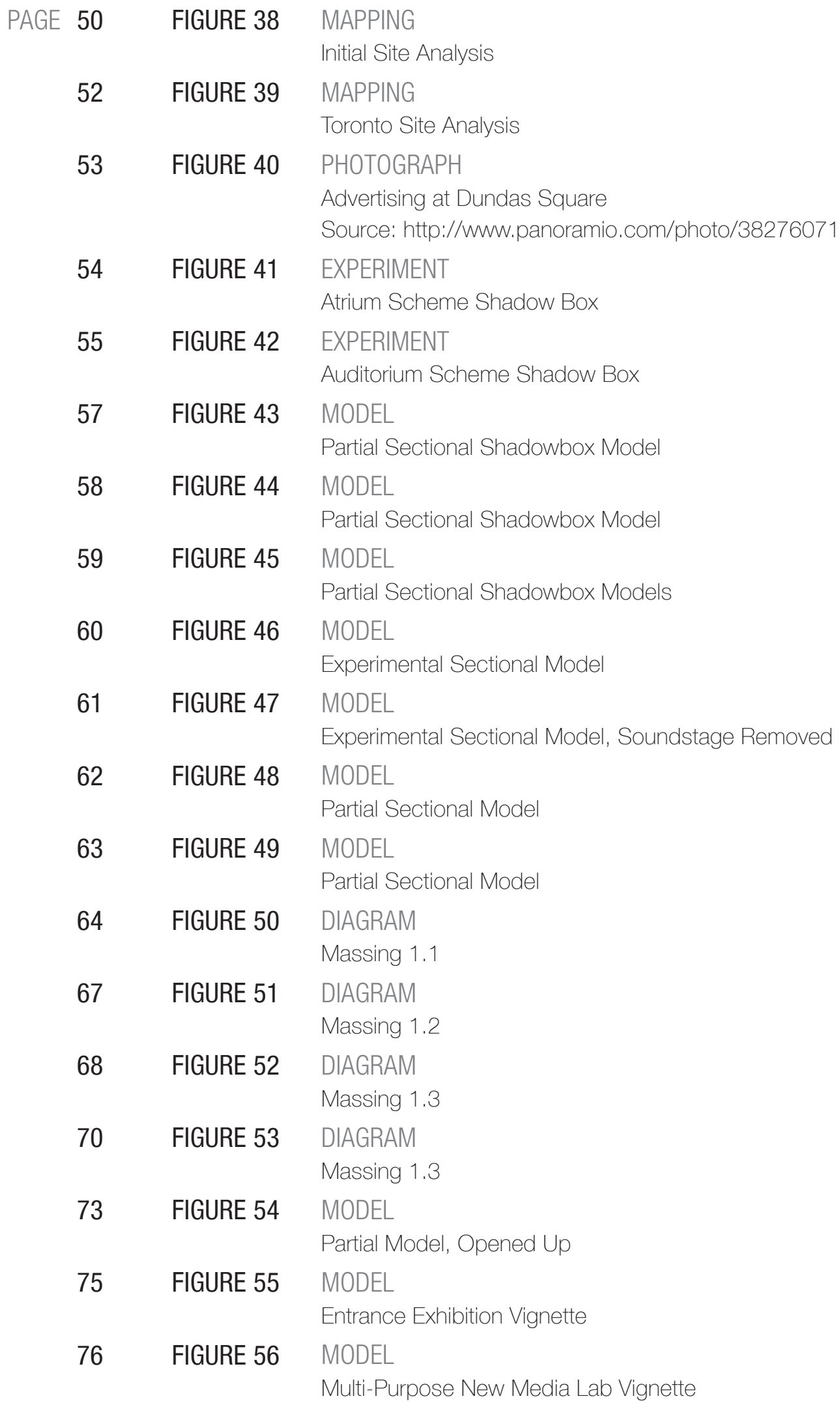


$\begin{array}{lll}\text { PAGE } 77 & \text { FIGURE } 57 & \text { MODEL } \\ & & \text { Multi-Purpose New Media Lab Vignette }\end{array}$

79 FIGURE 58 MODEL

Unfolded Circulatory Narrative Experiment

80 FIGURE 59 MAPPING

Design Iteration 1 \& 2 Sites

82 FIGURE 60 COLLAGE

Visual Narrative

85 FIGURE 61 EXPERIMENT

Exterior Views of Model

87 FIGURE 62 EXPERIMENT

Narrative through space projected onto Sectional Model

88 FIGURE 64 EXPERIMENT

Projection Testing on top of Model

88 FIGURE 63 EXPERIMENT

Interior/ Exterior Views of Model

95 FIGURE 65 DRAWING

Design Iteration 1 Basement Plan

96 FIGURE 66 DRAWING

Design Iteration 1 Ground Floor Plan

97 FIGURE 67 DRAWING

Design Iteration 1 Second Floor Plan

98 FIGURE 68 DRAWING

Design Iteration 1 Third Floor Plan

99 FIGURE 69 DRAWING

Design Iteration 1 Fourth Floor Plan

100 FIGURE 70 DRAWING

Design Iteration 1 Fifth Floor Plan

101 FIGURE 71 REFERENCE DRAWING

CBC Headquarters, Toronto

Source: http://www.cbc.radio-canada.ca/en/explore/who-we-arewhat-we-do/canadian/

102 FIGURE 72 REFERENCE DRAWING

CBC Headquarters, Toronto

Source: http://www.cbc.radio-canada.ca/en/explore/who-we-arewhat-we-do/canadian/

103 FIGURE 73 REFERENCE DRAWING

CBC Headquarters, Toronto

Source: http://www.cbc.radio-canada.ca/en/explore/who-we-arewhat-we-do/canadian/ 



\section{INTRODUCTION \\ MISE-EN-SCENE}

The 21st century has provided viewers with an inconsumable mesh of content, exponentially expanding and forever broadening in depth. The rapid speed of information exchange has begun to "flatten(ing) all communication onto a single, homogeneous surface..." (Calvino, 1988, p. 45). The sheer momentum behind the dissemination of images dislocates the differences and minute qualities of the work, serializing them into a mesh of similarities. The shifting focus onto the image of the artifact begins to remove the ancillary weight attributed to it. This effectively grants works of impressive magnitude moments of [un] divided attention to communicate their purpose before they are again lost into an abyss of competing images.

Architecture ceased to be a mere medium of building the moment it required justification. Inversely, buildings are merely one means by which architecture attempts to transmit information. All simulations of architecture, whether they be physical realizations or abstracted constructs, act to disseminate intent and are only realized through their desire to be interpreted. The architectural artifact gains weight only through interpretation. The mass consumption of images prevalent in the $21 \mathrm{st}$ century, forces artifacts to become largely self-referential and strips them of their ability to be positioned alongside a contextual lineage.

Context, or the lack thereof, has remained the constant in artifact dissemination and production. With little reliability afforded by context to the designers, works have begun to embrace their displacement. Each piece has become as "self-contained and independent of the context of reception as possible," (Mitchell, 2005, p.13) allowing it to harmoniously blend with any surrounding. Although the architectural artifact is heavily rooted in place, reproducible simulations remain in a realm of contextual ambiguity. The dual nature of artifacts is forcing the two facets to erode their differences in a chameleon like homogeneity, allowing the mise-en-scene to become as interchangeable as the artifact itself. 
Weight is the overt embrace of the interconnected web of relationships that produces, a voluminous tome to transcribe a work, anchoring the artifact to a body of text. This mise-en-scene moors the artifact with a tension or unease that can only be alleviated when the narrative moves, allowing the new space to absorb the ancillary layers of a grounded reality. This forces the designer "to change [his or her] approach, to look at the world from a different perspective, with a different logic and with fresh methods of cognition and verification" (Calvino, 1988, p.7).

It is not my desire to remove all weight; but to alleviate some of its necessity, allowing the weight to fluidly pass from scene to scene. To break apart the vastness and solidity of the encyclopedia and in turn focus on the particles which constitute it. This allows the particles to flow through the scenarios and realize themselves in a multitude of ways, all while being able to at a moment's note shed their cumulative weight.

The question at this point refers to the allocation of weight in the architectural artifact. One must simultaneously position the artifact between a state of utmost flatness idealized in the present condition without negating the necessity inherent by the burden of its own weight. To become flat, artifacts are separated from the meaning attributed to them by the designer. Without the narrative to rely upon artifacts lose much of their magnitude, transitioning into a caricature of their former selves. The primary obstacle facing the architectural artifact is the narrative sequence which fluidly shifts weight. Once divorced from the sequence the individual frames lose the weight attributed to them by the flow. A definitive structure sifts the individual elements into the most applicable position, carefully situating them amongst a series of similar elements to evenly distribute their weight. Each element at this point is suppressed, crafted in a manner which severs its undecidability.

The elements survival is dependent on the elaborately woven connection to the entire narrative, which begins to fray at its boundaries. The point at which the boundary is witnessed, the weave expands to encompass the additional facets to supplement the repression of the existing. Calvino noted this phenomenon in the works of his predecessors as they began to document the network of relationships associated to particular artifacts, in turn producing unfinished 'encyclopedic' texts (Calvino, 1988, p.107). In vain attempts at encompassing the multitude of facets, each exploration swells in the middle; perpetually battling at the fringe to prevent the fray and ultimately unable of returning to the origin.

Weight at this point should not be fluidly transitioning between artifacts to eventually run flat, but exist in a way which it is distributed amongst the particular facets. In place of the rhizomatic suffocation, is a desire for individual boundaries and contradiction allowing each facet to remain detached. 
Each investigation is afforded a tabula rasa allowing it to isolate a separate and seemingly disparate perspective into 'wholeness'. Instead of a birth through a translation of narrative, the physical artifacts themselves become structures keen on exemplifying their weight. Without the need to conform to an implied narrative, each facet is able to explore individual boundaries, becoming standalone narratives. By constructing parts with no definitive whole, each particle is capable of enunciating its own intent, while cumulatively constructing an ambiguous identity which is modular and combinatory (Calvino, 1988, p.120). 


\section{CHAPTER 1}

\section{THESIS STRUCTURE}

The thesis is structured along the notion that weight should be embedded within each artifact and not simply transferred through a narrative. Each facet is developed as a stand-alone exploration into a particular concept or thought process relating to the nature of the architectural artifact in the 21st century and the development of a New Media Production Centre for Ryerson University. Since the scope of each facet is loosely defined, manifesting itself in a variety of mediums, they are capable of investigating a wide breadth of subject matter. The aim of such an exploration is to allow each facet to develop itself without narrative conformity and readily be able to be situated alongside disparate facets. This in turn will provide an account that is inherently incomplete, possessing the ability to embody an array of minor narratives, and whose cumulative definition remains ambiguous.

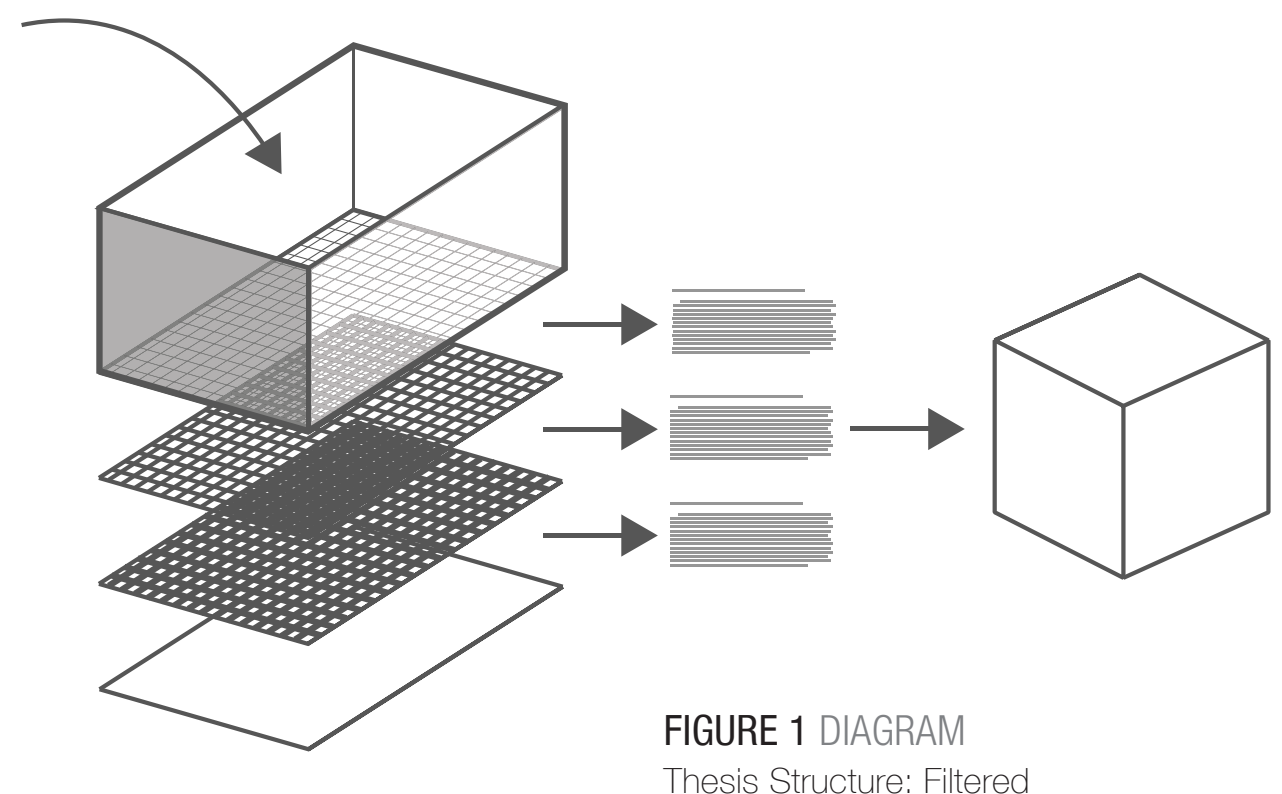




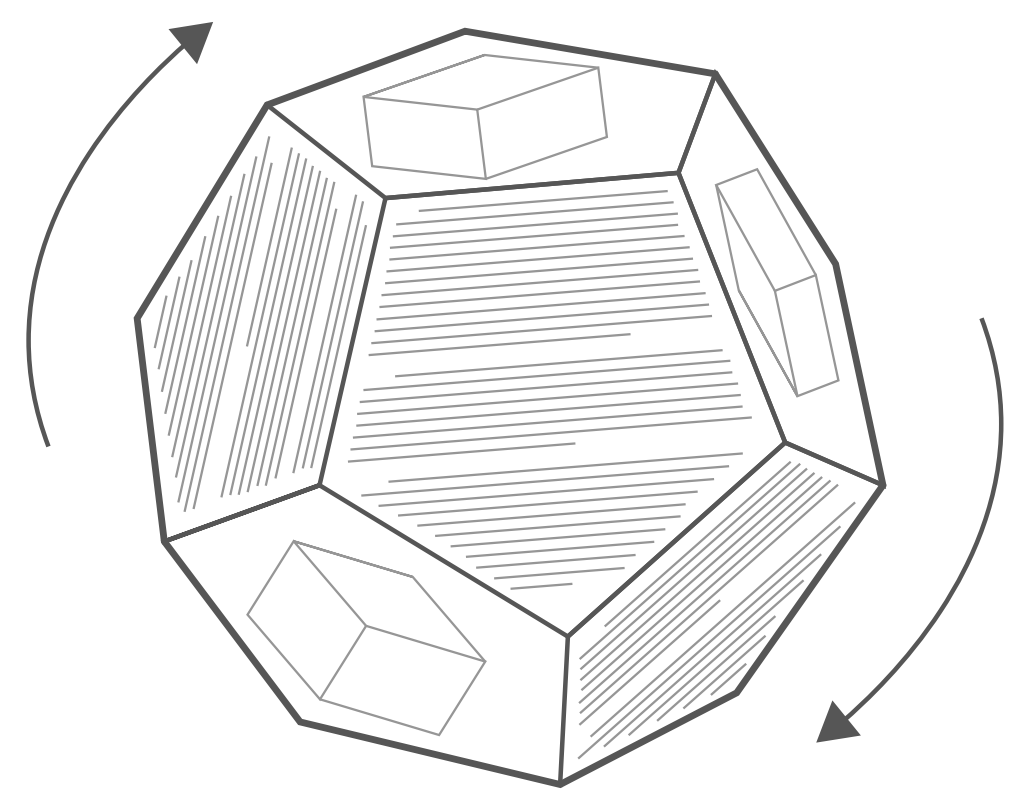

FIGURE 2 DIAGRAM

Thesis Structure: Multi-Faceted
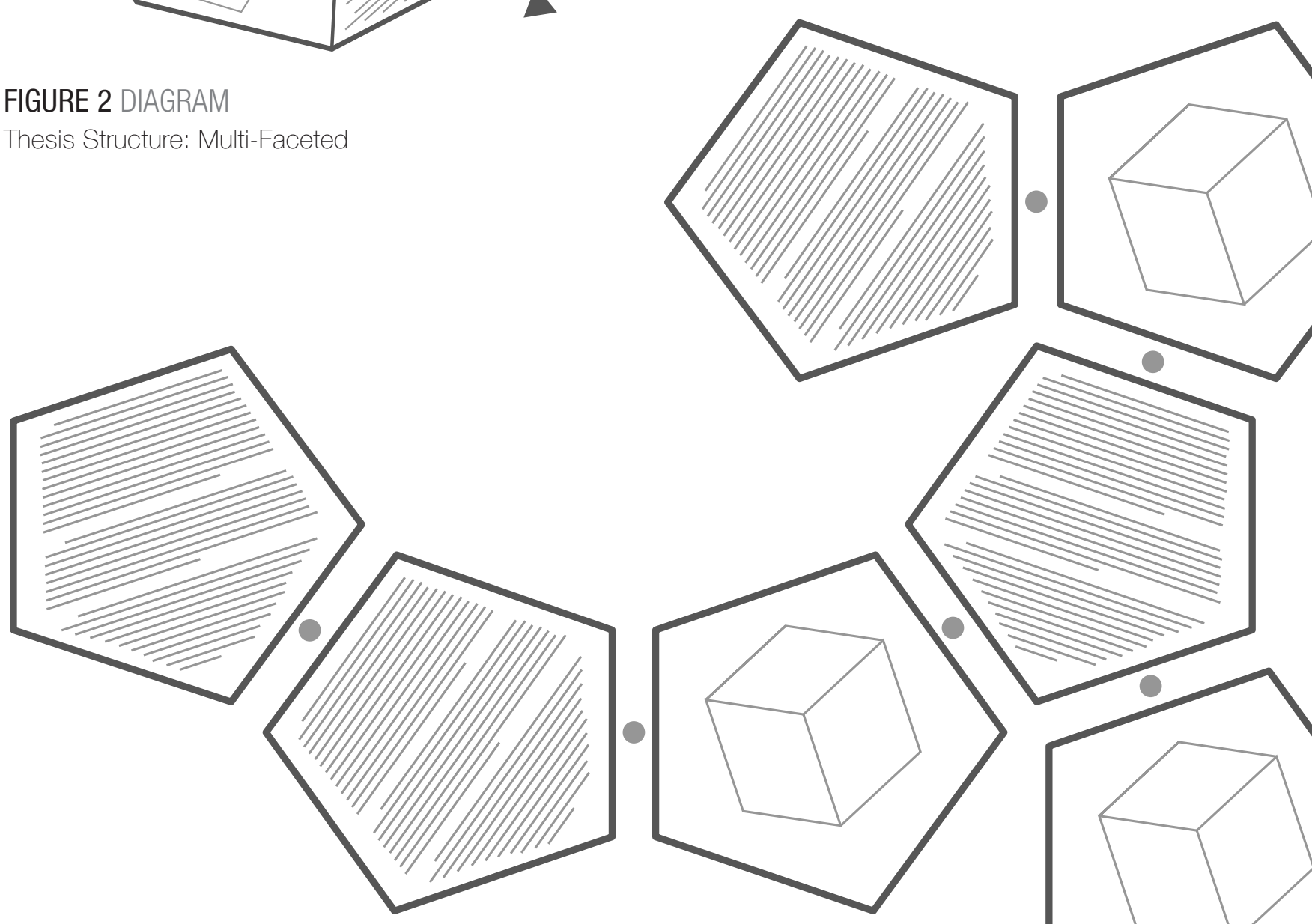

FIGURE 3 DIAGRAM

Thesis Structure: Multi-Faceted Unfolded 
The thesis and structure is born of a rejection of a more conventional structure which is focused on the filtering of concepts to be organized into a master narrative. Ideas are sorted into their respective chapters where their definition acutely conforms to the main message presented. In an objection to this convention a series of fragments are explored in a wider breadth of seemingly relatable topics in a nonsequential order. Each facet aims to investigate rhizomatically the development of a New Media Production Centre at Ryerson University while creating stand-alone entities.

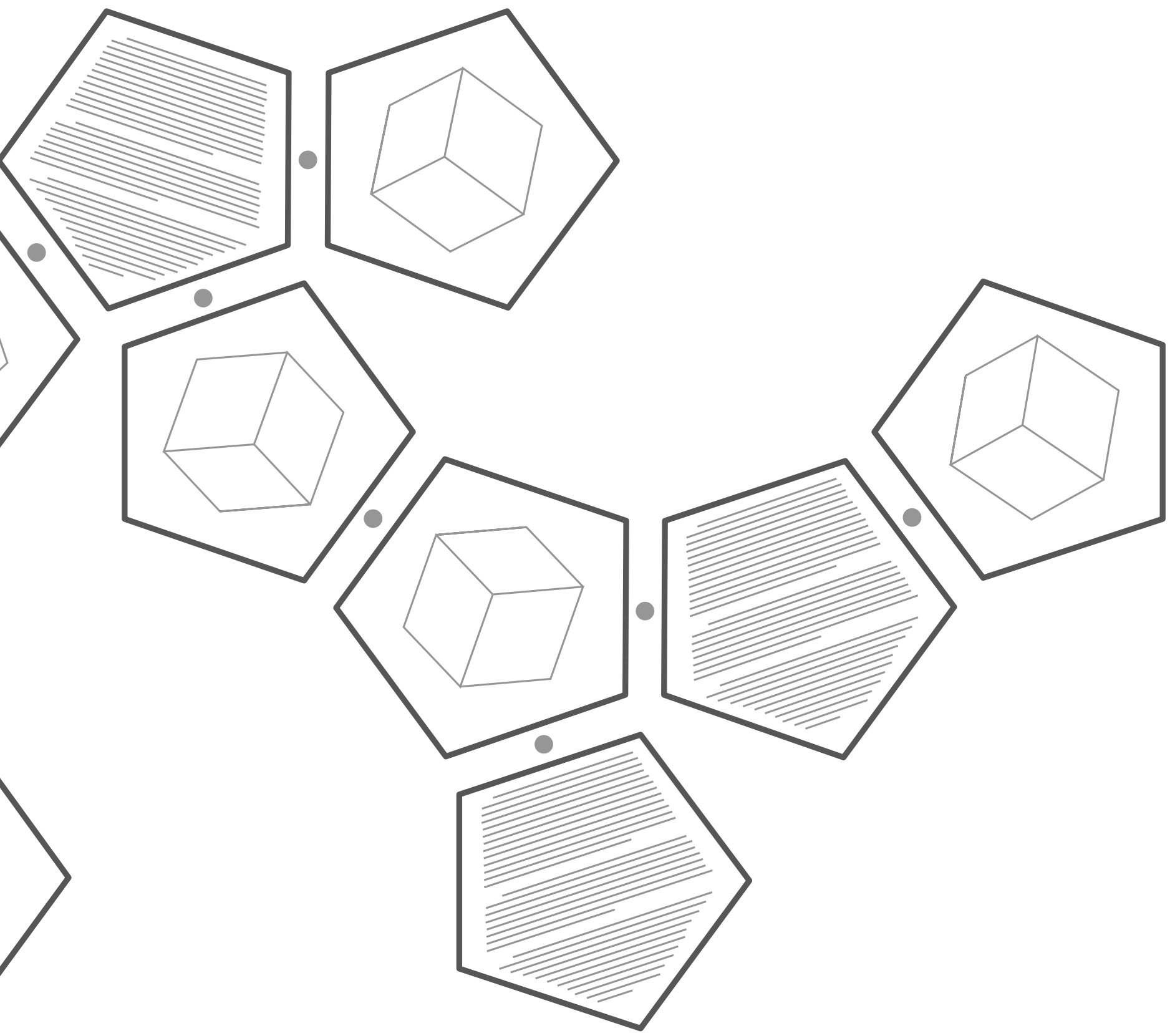




\section{CHAPTER 2}

\section{THE BEAUTIFUL UNCERTAINTY OF PIECES}

Jean Baudrillard in his 1981 philosophical treatise Simulation and Simulacra presupposes a condition where fiction and reality have collapsed onto one another, effectively dissolving the capacity for an absolute truth (Baudrillard, 1994, p. 11). Famously drawing parallels to former United States president Richard Nixon and the Watergate incident, Baudrillard emphasizes that a master narrative does not exist. However, what does remain is a constellation of events whose perspective constantly shifts based on the storytellers bias. To say that Watergate is a scandal for example is to instantly apply an opportunistic moral narrative upon the situation that is ultimately driven by an undertone of capitalism. In the same key-stokes which vilify Nixon, the journalists simultaneously are able to develop a parallel narrative of a president under attack from the media, whose escalating careers are merely a by-product.

Each facet behaves at an atomic level, where the aggregation of similar elements allows them to come together to establish a unique story. Additionally, the dissolution and subsequent restructuring of the atoms redefines the narrative that is produced. In Baidrillard's collapse of the fictional onto reality, the flattened state becomes one of inquisition. Each atom is understood in relation to the constellational web developed around it, however parallel, tangential or contradictory leaving the onus on the viewer to actively pursue each facet.

The 21st Century Museum of Contemporary Art by SANAA questions the form of atomization. Structured along an irregular grid, each gallery space fluidly establishes a relationship with the next. This configuration allows each gallery to behave discreetly or complementary to the surrounding spaces as required by the curators' narrative. The stunted cylindrical form of the 21 st Century Museum at this point 


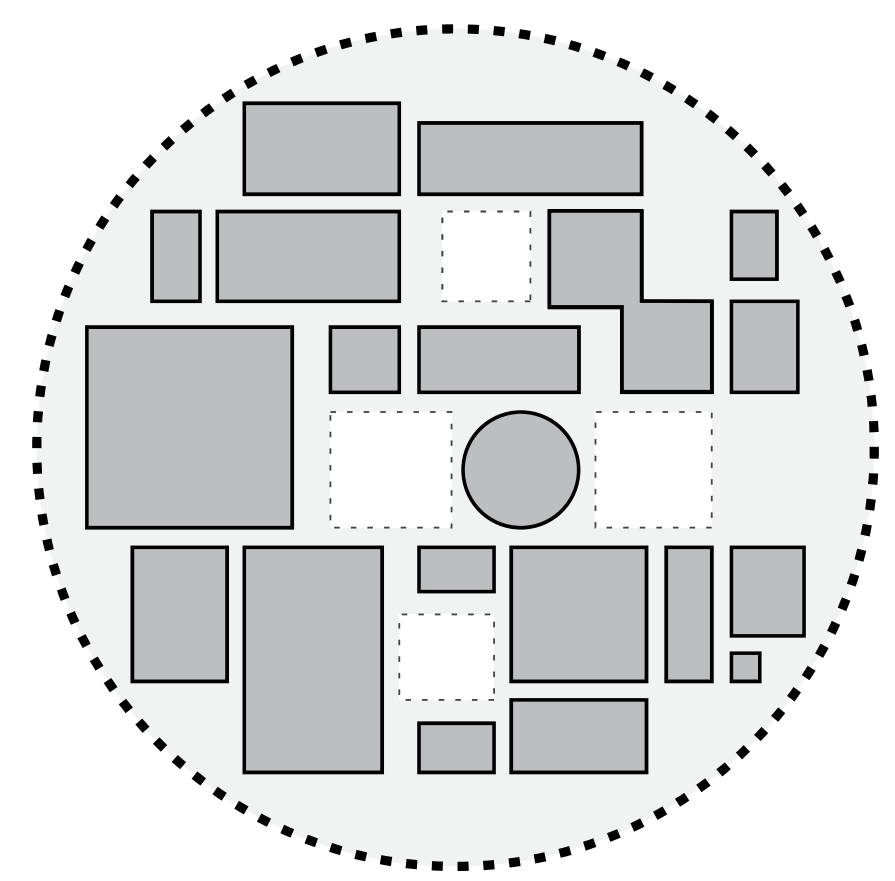

FIGURE 4 DIAGRAM

21st Century Museum of Contemporary Art - SANAA - Fragment to boundary analysis

is seemingly arbitrary, as the primary concern is to encompass the variety of atoms to unify them. The juxtaposition between the cube like galleries and the cylindrical can establish itself as a commentary between atoms and a whole. The whole in this case is a meta-narrative whose relationship is pursued on an agenda secondary to the individual gallery spaces and whose purpose is to legitimize itself.

In light of a view which embraces the inherent multi-facetedness of atoms, Italo Calvino closes his Six Memos for the Next Millennium with a commentary on the form of such narratives. In vain attempts to capture the radiating essence of such narratives, Italo Calvino notes that his predecessors are trapped in an encyclopedic formula; starting with an exploration into a subject only to find itself running simultaneously in every direction, unable to return to an end (Calvino, 1988, p. 116). The form simply expands from the middle, attempting to capture each radiating thread to determine "a network of connections between events, places and things of the world" (Calvino, 1988 p.105). By trying to document every facet of the conditions which influence the initial subject, the works become lost in their own exploration and are not capable of returning to the point of equilibrium.

The transformation of the narrative to encyclopedia, highlighting interconnectivity, gave the works an unbearable weight, causing it to abruptly crash under its finiteness. In place of a structure predicated on 


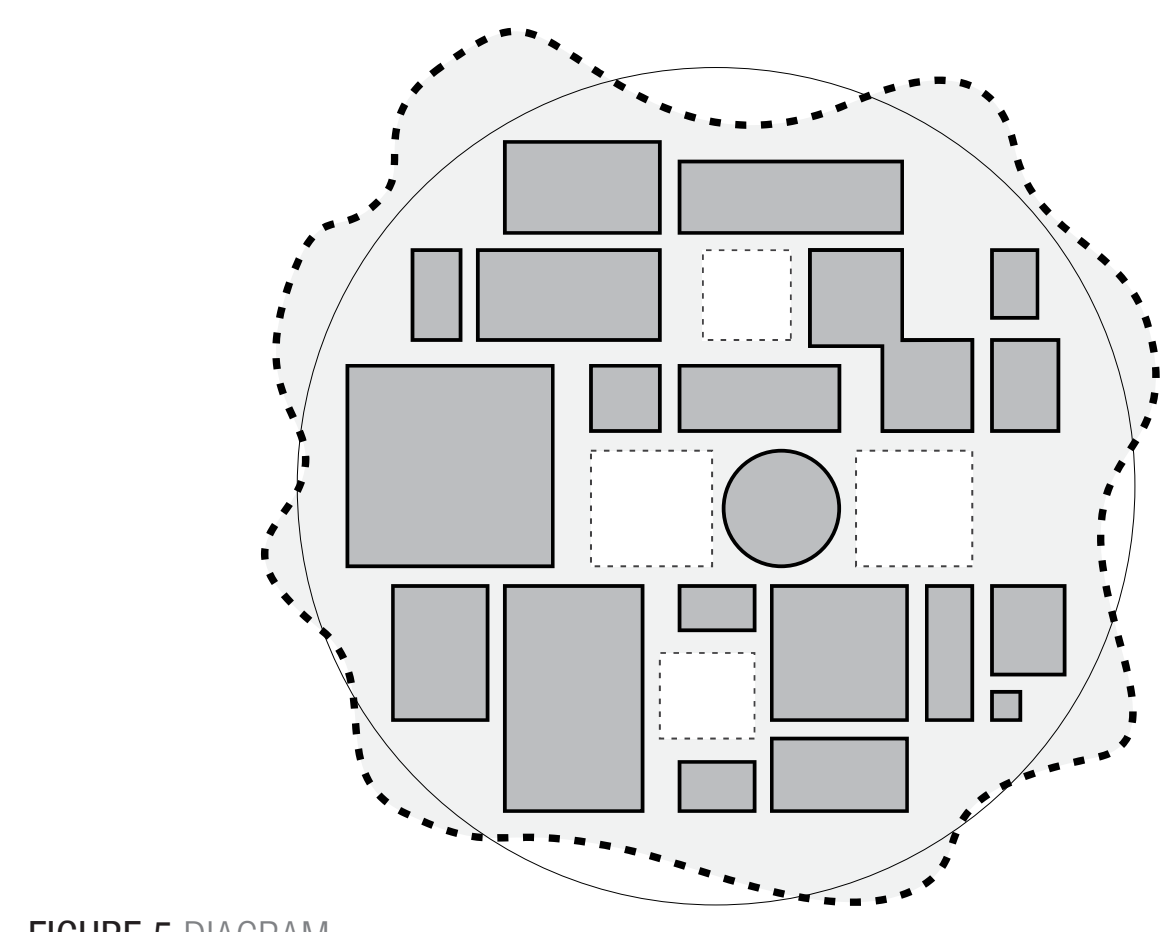

FIGURE 5 DIAGRAM

21 st Century Museum of Contemporary Art - SANAA - Fragment to boundary analysis

finitude, Calvino proposes that the contemporary narrative embraces uncertainty to alleviate weight. While the antiquated formula relies on a composed linearity, the contemporary narrative is inherently atomic; consisting of a series of isolated facets behaving hyper-real to their condition. By capturing particular essences of an artifact instead of the artifact in its entirety, a narrative structure emerges "which is accumulative, modular, and combinatory" (Calvino, 1988, p.120). Each piece at this point is capable of standing on its own merit, and whose understanding is altered with the injection of an endless amount of supplementary facets to derive the meta-narrative. The significance of atomization is the inability to succinctly capture all aspects of a piece, forcing it to focus on particular element as to no dilute the message. This allows the atoms to carry a very potent message and thrive in the uncertainty afforded to it. The form of such a structure begins to parallel that of SANAA's 21st Century Museum of Contemporary Art. Focus is placed on each gallery to be able to communicate the ideas it wishes to express. This composition is causes the narrative to be derived internally through experience, allowing the form to adapt to a secondary narrative disparate from the ever changing original. The cylindrical form merely establishes the boundaries as would the covers of a book, leaving one to explore the carefully manufactured internal network so they "can follow multiple routes, and draw multiple, ramified conclusions" (Calvino, 1988, p.74). 
FIGURE 6 PAINTING

Fighting Temeraire - Turner

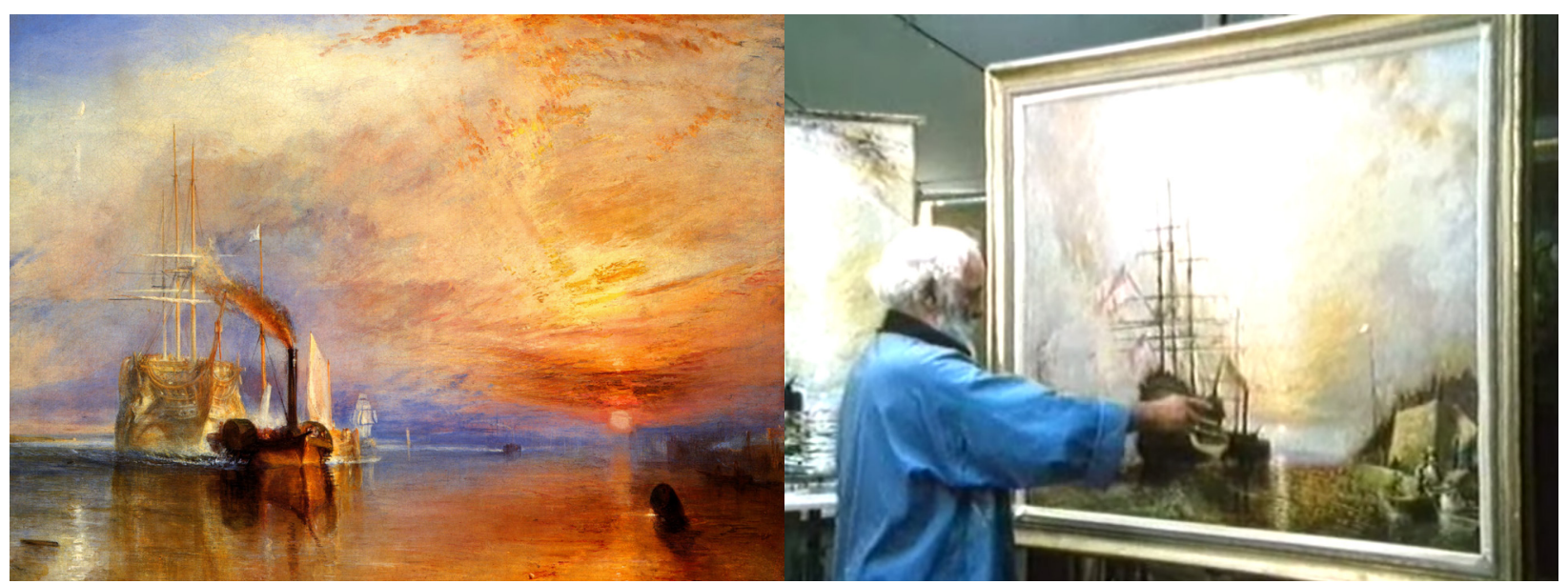

FIGURE 7 FILM

Still from "Tom Keating on Painters - Keating" 


\section{CHAPTER 3}

\section{THE SPACE BETWEEN STROKES}

A painting, much like the other communicative media, is able to express vast bodies of information with each stroke. The purpose and intent embedded within the process uniquely affects the character of the finished product. From the types of paints to the variety of application processes, a painter is able to individually inscribe a masterpiece with the epoch of their surroundings and revealing how they see themselves in that world. Unlike more contemporary media, the process of formalizing concepts in paint is instrumental in understanding the piece itself. Masters would often teach pupils their techniques, making difficult assessments of authenticity for present day art curators and evaluators.

Similar to method actors, Tom Keating (1917-1984) a renowned British painter and forger, taught himself to embody the artists he wished to copy. Although at times boldly criticizing the antiquated regime for which he painted through parody, the nature of his reproductions require him to completely step inside the mental space of the artist to relive the creation (Forged, 151). Keating is able to break the flatness of a piece by inserting, at times, himself into the work. Of particular importance is his recreation of Turner's Fighting Temeraire where the internal perspective is obscured to reveal the shore from which Turner painted the original (Forged, 155). Not only employing the process of production but physically altering the composition, Keating establishes a sense of hyper reality in his work unseen in the original.

Keating's captioned piece provokes a question of exposure. The relative ease with which he is capable to alter the work through his understanding of process questions the composition and rigour of the original; infusing the two pieces with a symbiotic connectivity that simultaneously facets the works. Although Keating's practice at times parodies the original he is replicating, the pieces are always scrupulously faceting the original to derive supplementary meta-narratives. 


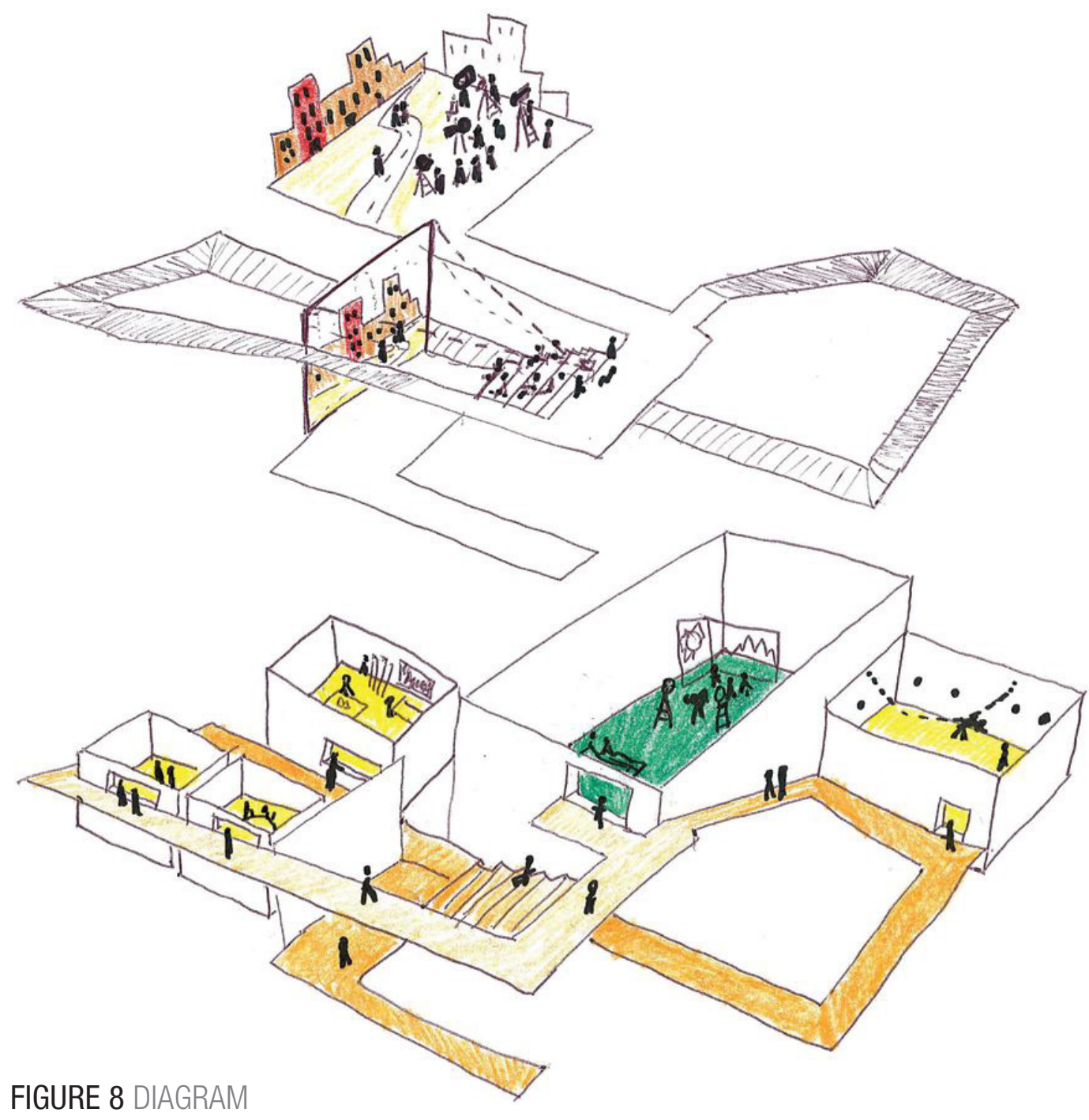

Columbia College Chicago Media Production Centre - Studio Gang - Circulation Diagram

Unfolding the flatness of film through process is the primary driver of Studio Gang's Columbia College Chicago Media Production Centre. The Studio Gang design utilizes the production process, structured along a linear narrative, to teach students and visitors about the various stages of film design. Beginning with a large projection in the entrance vestibule, the media production centre is able to showcase the students' work and through the subsequent journey through the facilities, develop a deep understanding for how the artifacts came into fruition. The master narrative of the centre weaves circulation through the various post production labs and studios, cumulating in the rawest output, the original film stage. The demystification of process grants the viewers an introspective glance at each of the post-production filters applied to the raw piece. By separating the individual production process, it draws attention to each layer of abstraction applied to the raw work and their effect in producing the desired affect. 
To question architecture's capacity for influencing the reception of an artifact is to investigate exposure. In film exposure refers to the amount of light which is able to reach the film as determined by the shutter speed and lens aperture which are limiting conditions. To further this, exposure can be understood as the revelation of a facet, in particular facets which may be concealed or initially dismissed. Architecture, in the highly pragmatic sense witnessed at the Columbia College Chicago Media Production Centre, manipulates a programmatic exposure to emphasize the elements of cinematography that are easily overlooked. Through the structuring of a programme and the establishment of a sequential narrative structure, Jeanne Gang forces over exposure onto a viewer. The succession of spaces additively develops a narrative to parallel the one presented in the entrance.

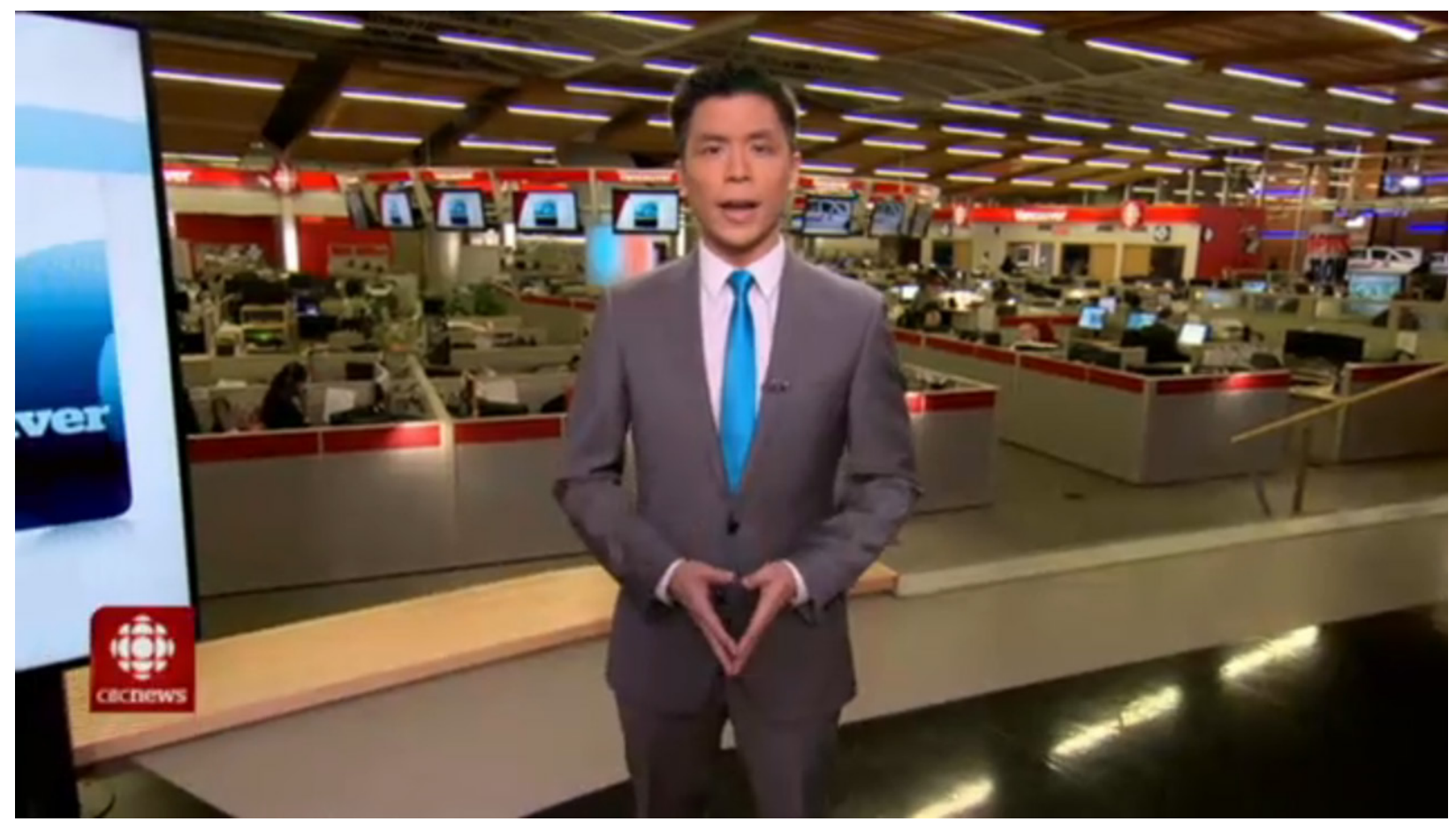

FIGURE 9 FILM

Still from CBC News Vancouver @ 6

This notion is furthered in the Dialog redevelopment of the CBC Broadcasting Headquarters in Vancouver, Canada. Of particular importance is the relationship between the daily news broadcasting desk to the staff. Traditionally the flow of information is not fluid, and is often separated through several barriers. In a large open space, reporters are able to collaboratively work on stories and gather information which is then filtered into a control room. The control room, which exerts authority over the news desk, further processes the information and transcribes the teleprompter to be read by the anchor. The final transmission is one of utmost control, where every aspect becomes highly regulated and overseen; absolutely removing the process which led to its formation. 
The uniqueness of the Dialog design is the spatial relationship between the news desk and space for reporters. The news desk is located in the corner, backing onto the street where a passerby occasionally engages with the anchors. Two semi-transparent walls, upon which television screens hang, establish the spatial boundaries creating a large gap in the centre spilling out directly into the reporters' area. The camera is operated from the centre of the space, allowing the anchors to freely move along the boundaries and interact with the reporters behind them. However gestural the maneuver may be, it affords the viewer glimpses into the news process, a statement emphasized by the news area's floor to ceiling glass windows along a pedestrian heavy street. The scheme democratizes news by demystifying the process and visually including everyone in the broadcast.

Exposure must consistently challenge what is shown to expand, or at times contract what is shown. The two spatial tactics heighten the narrative by asking the viewer to become aware of the process. Behaving similarly to Keating's imitations, the perspective of the traditional space is changed to incorporate a parallel narrative; forcing upon the viewer an additive understanding that runs congruently to the original. 


\title{
CHAPTER 4
}

\section{REMIX AND THE 21ST CENTURY ARTIFACT \\ "...life itself is a fiction and biography is something one invent afterwards." \\ Mark Amerika in conversation with Eduardo Navas (19)}

\begin{abstract}
New media is a term coined to express the inability of traditional media to capture the epoch of the present condition. When the term first emerges in the late 1990s, new media emphasizes negativity towards the past while "envisioning the future as a realm of undefined, fluid, and hence unpredictable forces." (Koepnick \& McGlothlin, 2009, p. 2) New media is not limited to a singular medium, but instead the constellation of artifacts that emerge from technological advances.
\end{abstract}

We have become increasingly synonymous with new media as the technology that allows it proliferates, seemingly allowing the present to overwrite any history without it. "The task now is to ask students to imagine an era, not so long past, when the default perceptual norms were not videographic, when there were no expectations of interactivity with the image, and when screens were found principally in movie theaters." (Rodowick, 2007, p. 93) It is becoming increasingly difficult to comprehend the past without mobile devices and constant access to digital information.

The 21st century artifact is a direct reaction to the evolution of process and dissemination. The rapid development of technology is seeing constant permutations on the capture and delivery of media, in turn fundamentally altering the personal relationships with said media. Never before has one been able to shoot short videos, process photos, curate and observe media of increasing physical quality on a device a little larger than a packet of gum. Although the democratization of media, that is the decentralization of content production from large institutions to an individual scale, is regarded as reducing the overall quality or significance of outputs, (Stott, 2014) it is impossible to deny the aesthetic influences that continue to permutate into popular culture. 
An attitude running parallel to the advent of new media is that of the remix or the rehash. The notion of the remix, already prevalent in the sampling culture of contemporary music, has been slowly bleeding into our social sensibilities. Whether you draw similarities to the appropriation art of Marcel Duchamp, Andy Warhol, and their contemporaries, or to the use of citations and quoting often found in academic journals, the idea of remixing is already common practice (Manovich, 2007, p. 3). The act of remixing can be understood as the any networking of existing cultural works, or drawing together modules of cultural significance to articulate meaning. Authorship at this point becomes synonymous with proclamation, where the creator stakes claim to the meta-narrative derived from the combination of existing elements (Keats, 2012, p. 162).

Lev Manovich, in The Language of New Media posits that the creation of artifacts is predicated on the use of media elements. These media elements, embedded with a degree of cultural identifiably, are the images, sounds, shapes and behaviours that are sampled from the existing bodies of work we inhabit. Of key importance is that "these elements are assembled into larger-scale objects but they continue to maintain their separate identity. The objects themselves can be combined into even larger objects -- again, without losing their independence. (51) Artifacts at this point are constantly deconstructed and reconstructed as seen fit. This allows the individual elements to oscillate between various remixes and assume varied identities.

Artifacts currently exist as fragments or modules. The internet behaves similar to an archive or database, collecting the rapidly expanding collections of information into its storage facilities. Search algorithms, in an attempt to mitigate the sheer media overload, sift through content by most recent content and relevance. These two factors showcase two overlooked facets in content distribution.

First, in structuring the information based on date of input, media is reinforced as being fragmentary. All information is broken up into its smallest elements for easiest consumption. This focal shift complements everyday search patterns towards kernels of information and not entire bodies of information. The search algorithm presupposes subjects as already constructed from individual parts, providing users with an addendum for existing knowledge and a primer for further research (Navas, 2013, p. 22).

Second is the dominance of popularity. In an attempt to achieve the most relevant results information is structured along popular consensus. This begins to shatter lineage and original authorship in digital environments, furthering the legitimacy of the remix. A video for example such as the "Harlem shake" viral video can be posted to Youtube, and shortly afterwards numerous permutations exist in which the premise remains but new takes and scenes are introduced. Each variation is competing for the same space and 
traffic, with those (including the original) who are less successful slowly becoming lost into the internet abyss (Navas, 2013, p. 22).

Production in internet culture is also experiencing this fragmentation, shifting from highly designed information objects and in place focusing on "micro content" or easily distributable artifacts (Manovich, Remixability and Modularity, 2005, p. 6). This allows the collections to be readily broken down into their raw elements, allowing the evolving tools of dissemination to further aggregate them in new and useful ways. In order for digital content to survive it cannot be constricted to its source. What is more important

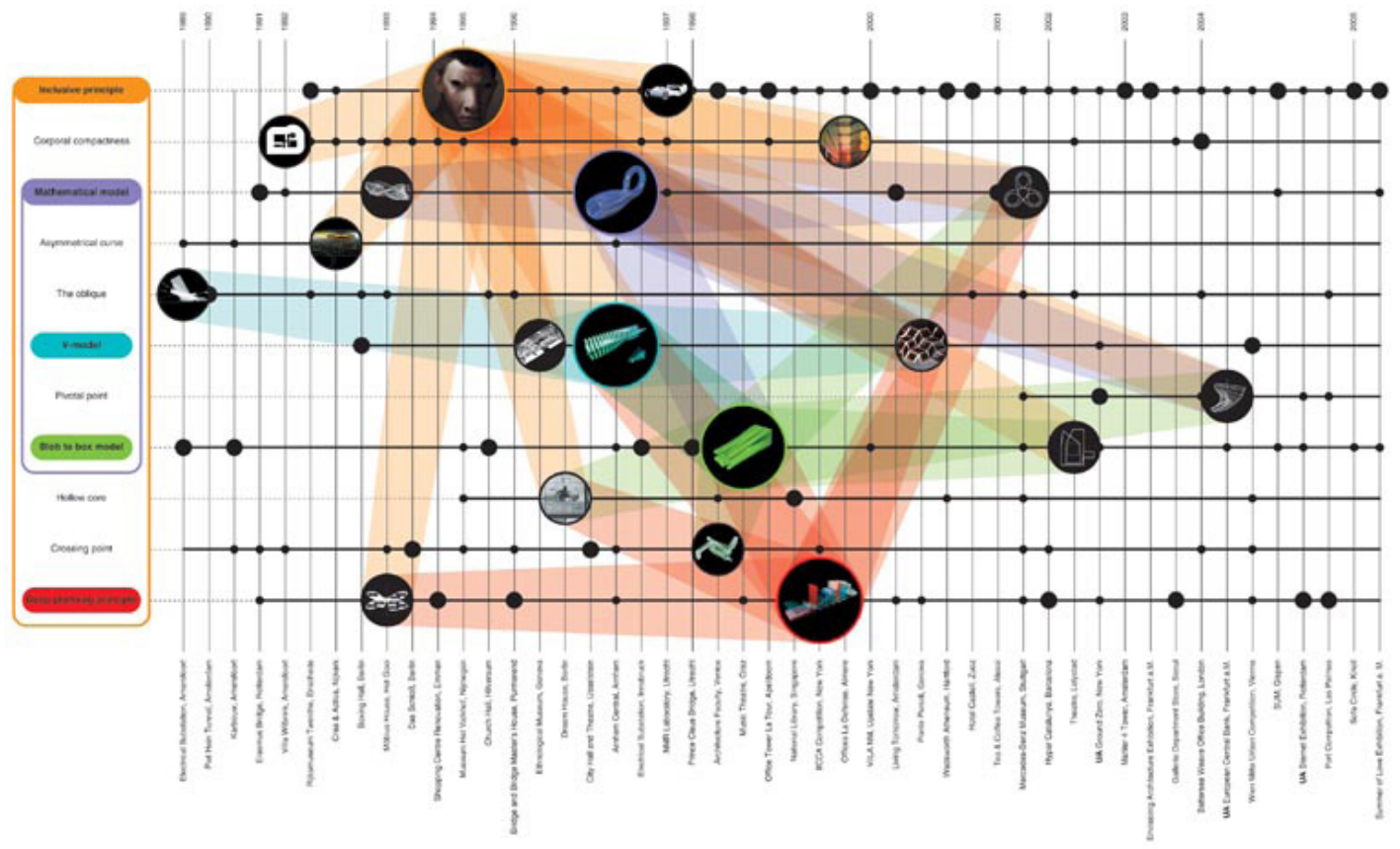

FIGURE 10 DIAGRAM

UN Studio Design Model

than the apparatuses such as ipads and video portals for the content, is the ability for media objects to travel between them, as they are only temporary stations in technology's perpetual evolution (Manovich, Remixability and Modularity, 2005, p. 7).

The design practice of UN Studio is beginning to apply the practice of remixing into their work. In an attempt to reject the typical project by project design, the focus has been on the creation of design models which can shift and develop in future projects. The focus of the design models in UN Studio is on seriality, or allowing their elements to constantly repeat and alter copy and move, allowing them to do the same but differently (Van Berkel \& Bos, 2006, p. 16). In understanding their work as "micro content", capable of stripping the superfluous information directed by site and context, the essence is capable of 
constantly permutating. "We see design models as packages of organizational or compositional principles, supplemented by constructional parameters. The design model does not include site-specific information; it exists at a more abstract level and may be implemented in various situations and projects." (Van Berkel \& Bos, 2006, p. 18)

New media and the 21st century artifacts that constitute it are tied to the digital advances that bring them into fruition. The digital realm thrives in the ability of artifacts to be modular and adaptive, often forcing them to do so simply to survive. Instead of rejecting the past, new media gives new life to the work that precedes it, recreating and contemporizing this content. The constant evolution of cultural artifacts speaks to an era of positive indecisiveness allowing fragments, who alone carry the essence of message, to be endlessly combined into works of significance 


\section{CHAPTER 5}

\section{SEQUENCE AND ILLUSION}

Cinema inherently relies on illusion. The success of film lies in its ability to lure the observer into a story, placing blinders over their eyes so they become immersed in the screen. In essence, a film is a collection of carefully framed scenes shown in succession. In the production of each scene, attention is brought forth to capture a particular moment or to progress the narrative. A facet of the success of immersion is the active participation of the observers, forcing them to consistently bridge gaps in the information presented in order to understand the work. This necessity to fill in the blanks, or trust in the act of deception allows a director to film in one location and with a few establishing shots of another, transport the viewer to that world.

The divide between given and implied information is further complicated by sequence in cinema. Examples of non-linear story telling such as Christopher Nolan's Memento (2000) in which a man with short term memory loss searches for his wife's killer, forcing the observer to constantly wipe any sense implied information. The story is unfolds itself in reverse, cutting to scenes set prior to the beginning of the film as the protagonist becomes aware of them. This is done to allow the protagonist and observer

FIGURE 11 FILM Film still from Memento (2000)

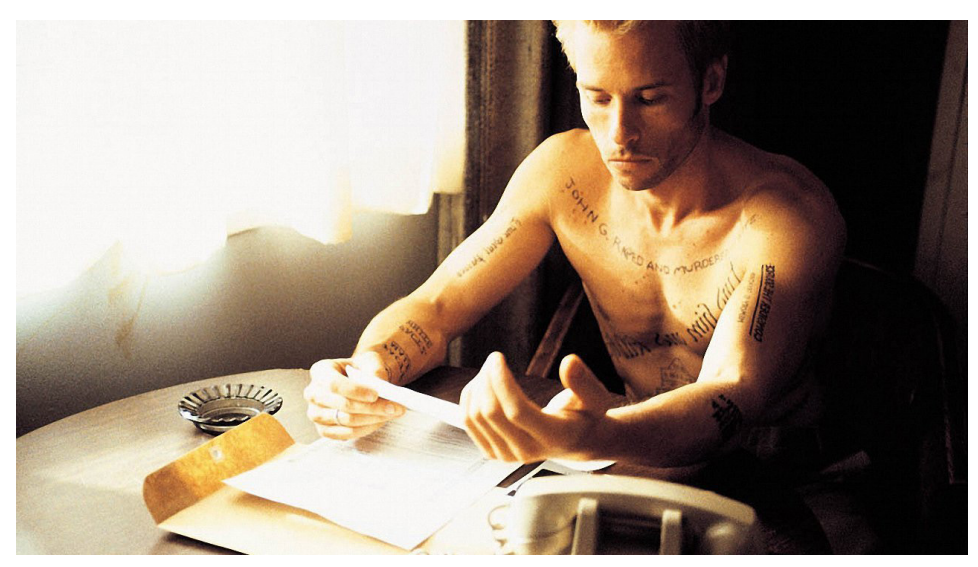


to consume information simultaneously, without giving one party a privileged perspective. Additionally this gesture produces two parallel story lines, of past and present, which converge by the end of the film.

Similarly Greg Marcks' 11:14 (2003) tries to capture the events of a car crash at 11:14pm that recounts the event from five different perspectives. Each pass at telling the story augments the observer's previous understanding, forcing them to question where the viewer might have implied information, instead of focusing on the facts. Both examples are effective in fragmenting narrative and structuring the story in a manner that challenges the observers given and implied understanding. The stories are broken down into a series of scenes that hide an element of truth within them, an element that can be searched for in subsequent viewings.

The phenomenon of constant questioning is achieved with post-processing. Every scene, or artifact, is carefully constructed to exemplify a particular condition. From the careful composition of elements in a frame to the way that the camera captures the actors, the director engages the medium directly to produce a hyper real fragment of the idea(s) being conveyed. Post-processing at this point becomes a conversation with the medium itself to learn how successful the artifact is capable in expressing itself, and then massaging the delivery to achieve the desired response. This conversation elevates the individual scenes and through the sequence questions the observer's interpretation of given and implied information.

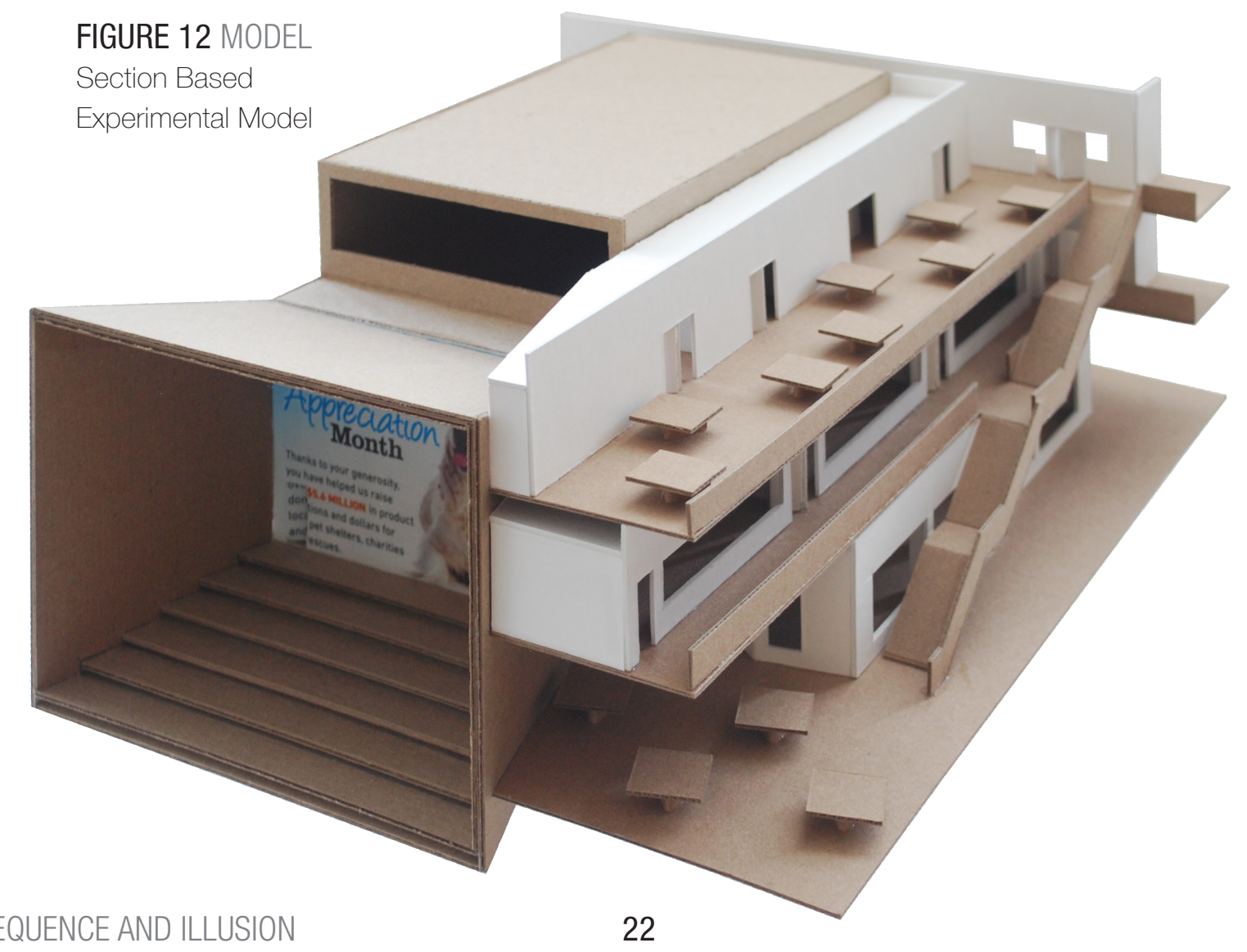


The importance of post-production shifts the bias from narrative producing artifacts to artifacts producing narrative. The preference of artifacts creating narrative requires a heightened restraint from implying a message through the artifact. Instead artifacts must be read blindly to catch derived associations, at which point the post-processing can direct how the viewer will be drawing conclusions (Kipnis, 2013, p. 225). When introduced in a sequence each artifact begins to frame and filter the artifact that follows it. This act leaves a faint trace of the artifact that precedes it, asking the observer to bridge a gap between the two.

The artifacts that are produced from narrative are constrained to the position they are derived from, effectively asking the message to explain the medium. With many digital forms of dissemination removing the control of delivery and separating the original message from the medium, the issue becomes compounded. The exposure of the fragmented composition of a work is a critical underlying theme in this thesis. An understanding of the necessity for post-production begins to highlight the additive nature of artifacts and fosters the development of several congruent narratives depending on the desired effect.

Once removed from the established context scenes in film must carry their own weight and truly exemplify their essence. In our age of context-less digital image consumption, the same can be said about the architectural artifact. When viewed in isolation it is imperative that the artifact can begin to stand for more than itself, allowing meaning to oscillate between its various interpretations. The section based experimental model not only begins to convey notions of spatial composition pertinent to the given requirements but attempt to define its own fragmented nature through the various section cuts and continued paths of travel. On one level the model readily allows the observer to comprehend the spatial implications of said gestures while simultaneously holding a conversation on a mental necessity to complete the narrative.

In holding a conversation directly between the designer and the artifacts, the creator begins to understand that the piece is expressing in comparison to what was initially desired. This discourse extrapolates a trajectory from the work itself, a trajectory which can be further understood though additional experimentation. The translation between idea and physical manifestation is rarely as direct as the designer would desire. Without developing the medium and message congruently the divide between the two will only grow more disparate.

The architectural artifact and scenes in cinema are constantly required to balance both identities. The message and communicative medium must work harmoniously. Without a message the communicative medium consists of nothing but a few jump cuts and fancy filters. The message justifies the tactics utilized to realize the artifact while inversely the tactics reinforce and over exemplify the message. Both facets are in a constant dialog with one another, massaging and redefining each other's parameters until a desirable output is created. 


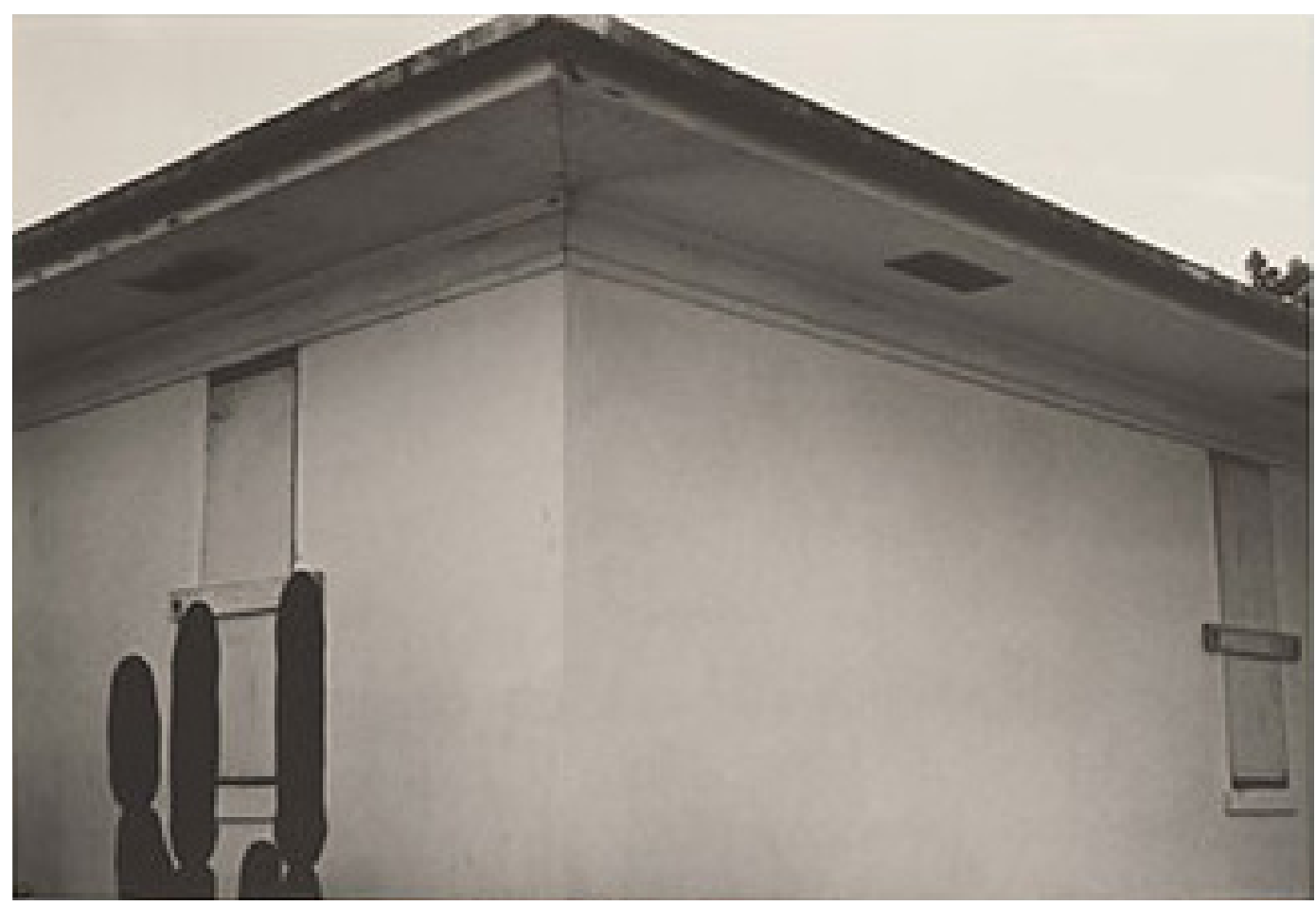

"They were tired of the house, so they hired some neighbourhood boys to board it up and make sure all was secure. He was 15 and figured it was a good way to make a few bucks. He brought Francine alone because they were going to the movies later and she thought she'd sit on the sand till he finished work. After about 2 hours she walked up to the house and watched them filling the windows with wood. She told him that when she was little this used to be her dream house. He hammered away, listened half-heartedly, and said "Oh yeah?" she and her friend used to sit on the bench across the street and just watch the house. Now the trees moved so slowly around it with each breeze. She would picture herself inside making breakfast. She is wearing a white apron which has printed on it a picture of little bees with smiles on their faces hoping from flower to flower. She is making French toast for her husband, who is a successful real estate broker. She tells this to him as he finishes boarding up the large bay window in the living room. He rolls his eyes in his head, says "really?", puts his arm around her and kisses her hard on the lips."

\section{FIGURE 13 PRECEDENT}

$\mathrm{A}+\mathrm{B}=\mathrm{C}$ Barbara Kruger (1978) 


\section{CHAPTER 6}

\section{DECONSTRUCTING ARTIFACT CONSTRUCTION}

\subsection{LAYERING}

The work of Barbara Kruger exposes the correlation between text and image. By positioning a mundane image of an often overlooked architectural detail alongside an elaborate, emotionally stimulating text the two become charged. The detail is now fueled by the ancillary conversation, drawing the viewer to examine even more minute details of the photograph. The text is simultaneously washed with an aura of the everyday, making the text more relatable. The piece questions the role that layering plays in the interpretation of a work, in particular how the relationship between the text and object is radically altered based on their successive placement.

The second iteration of a layered image interacts with the perception of a space. A communal break out space located at the Morphosis Graduate House for the University of Toronto is overlaid with a text based explanation. The meaning of the gesture is two-fold. At first glance the text establishes context and replaces the necessity of people in the rendering. The second reading exposes the fallacy of such a gesture. The space is ear marked as inherently communal with the intent of bringing together the various students while in reality it is empty. The layering of information at this point asks the image to carry all of its communicative weight, forcing the artifact to become self-aware.

Layering brings into question a certain degree of exposure with a work, particularly how a subtle shift in how information is presented can affect the perception of said work. By reversing convention as is

present in the layering of news production and dissemination of the Dialog CBC Headquarters Vancouver, designers are granted the possibility to alter spatial awareness. 


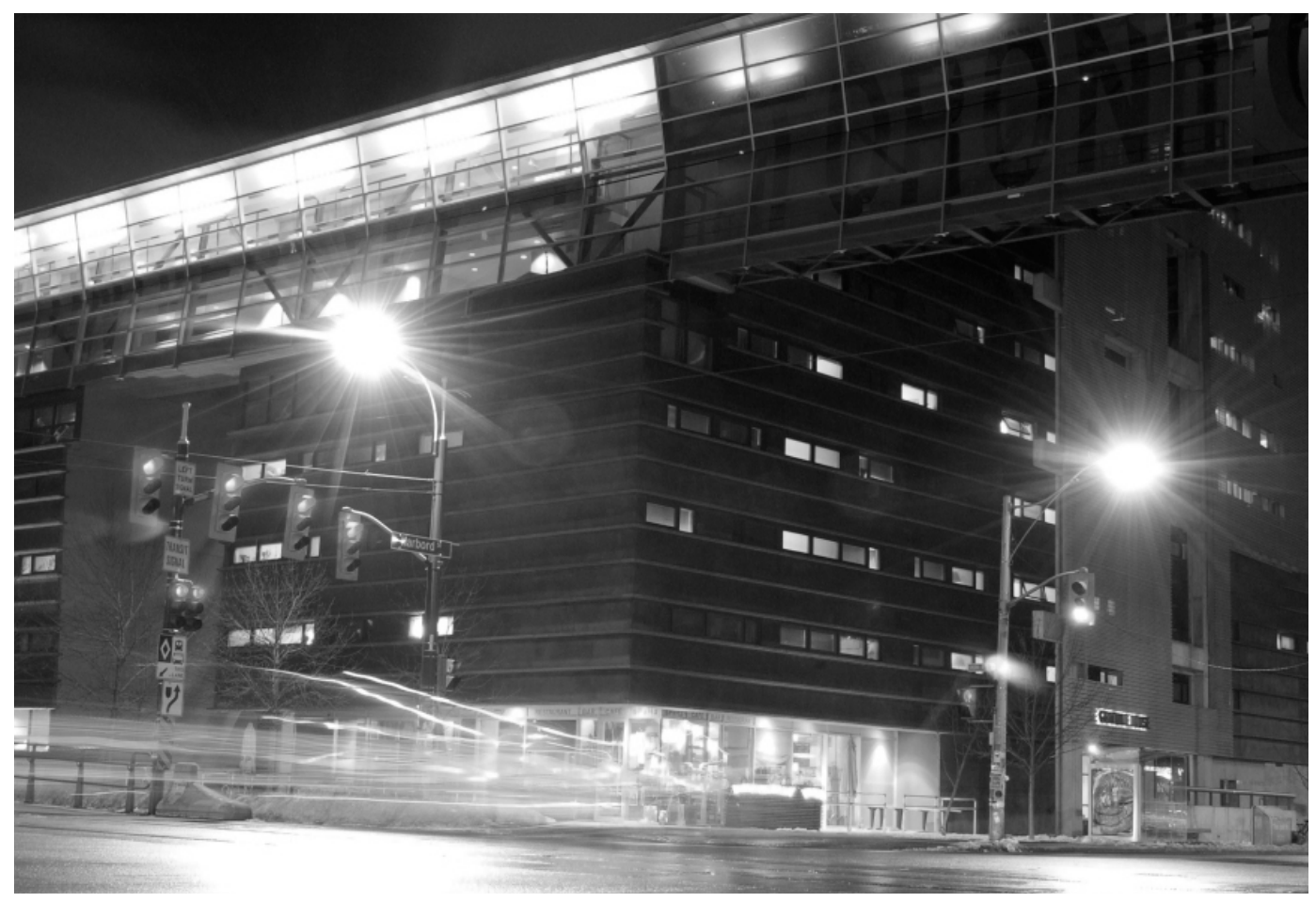

It was a perfect hybrid between research assignment, first date and trespassing.

After awkwardly standing at the side entrance smoking three gangly graduate students, each carrying a case of beer, open the door. Seeing our chance to break away from the cold we followed, losing them after a couple turns. The adventure ensued as we commented on the ridiculously low ceiling heights, spewing prototypical archibanter about the prestige of living in a Morphosis design. At the end of each corridor is a communal space more often than not devoid of students. We sit in armchairs browsing through the economic times, fingering the spines of dated books. A security guard making their rounds catches our guilty demeanors as we hastily hide our cameras. Moments later we are escorted through the main entrance.

FIGURE 14 EXPERIMENT

Illustrative Captioning 


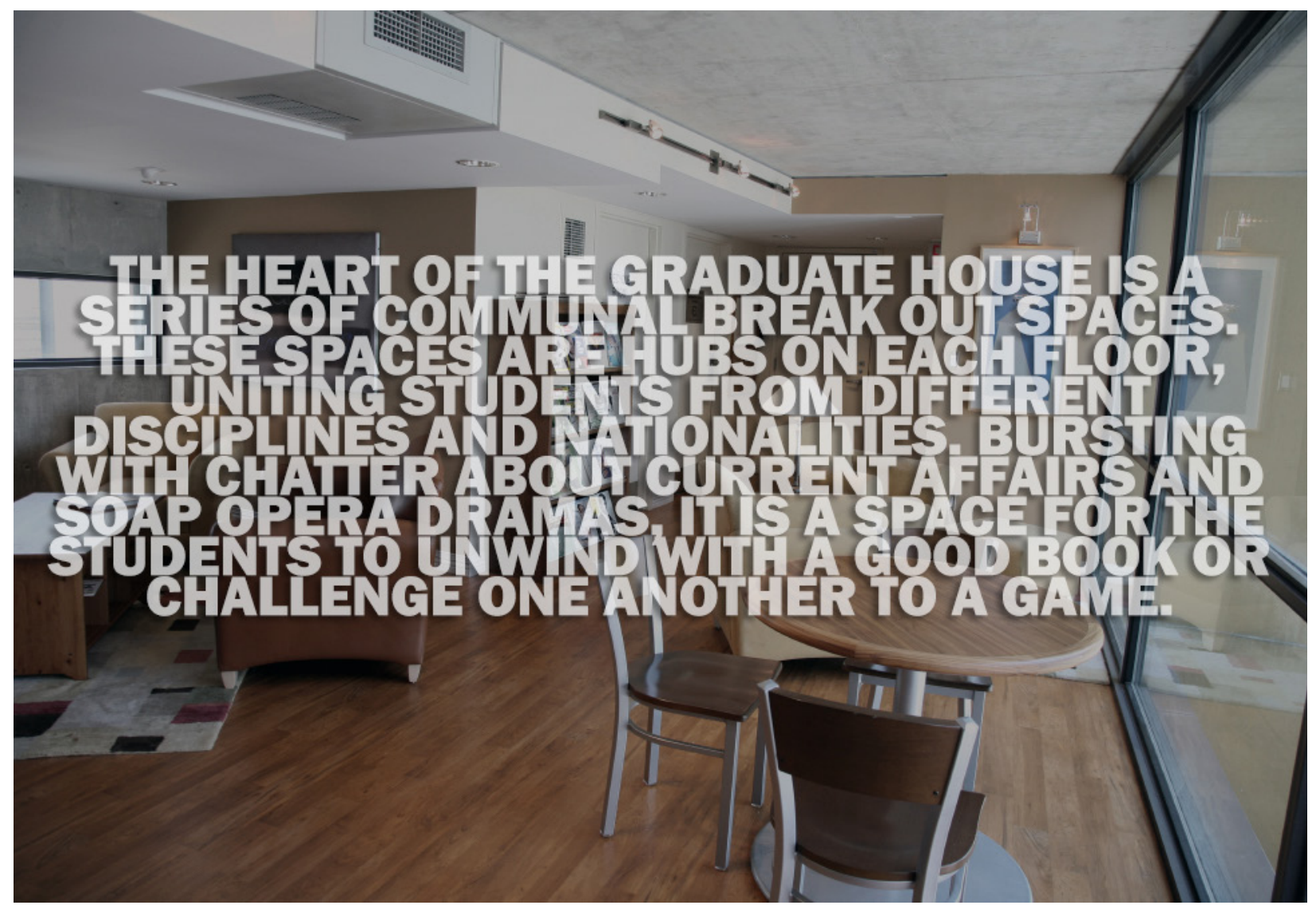

FIGURE 15 EXPERIMENT

Text Based Atmospheric Rendering 


\subsection{TRIPARTE DIAGRAM}

Following the narrative structure of 'The Manhattan Transcripts by Bernard Tschumi, the triparte diagrams aim to deconstruct popular advertisements. The Manhattan Transcripts saw a noir detective mystery broken down into a series of three part images consisting of a referencing image, plan, and movement diagram. The aim of the 'Constructing Advertising' experiment (Figure 16) is to dissect the narrative structure of each advertisement. The Ascent advertisement for 5 Gum sees an ambiguous story, slowly narrowing in direction until the purpose becomes evident. The narrative journey can be characterized as a tapering tunnel with a wide opening slowly transitioning into a limited perspective. Conversely The Closet for Canal+ draws the viewer down a series of misdirection's. As the viewer begins to posit the outcome they are soon taken along a tangential narrative. Finally the Honda Fit advert Clocks, Wrestlers, Creeps and Centaurs provides

the viewer with a series of vignettes focusing attention into a variety subjects, centralizing the observer in a seemingly endless amount of possibilities that are cumulative and highly modular.

The breaking down of the image into a series of associated focal points allows for a more holistic understanding of the term in question. The next exercise saw a continuation of the previous exploration using the different ways that narrative is manipulated architecturally focusing on folding, slicing, atomizing, distancing, and disorientation. Each term is explained verbally, diagrammatically, experientially and through the use of precedents. 


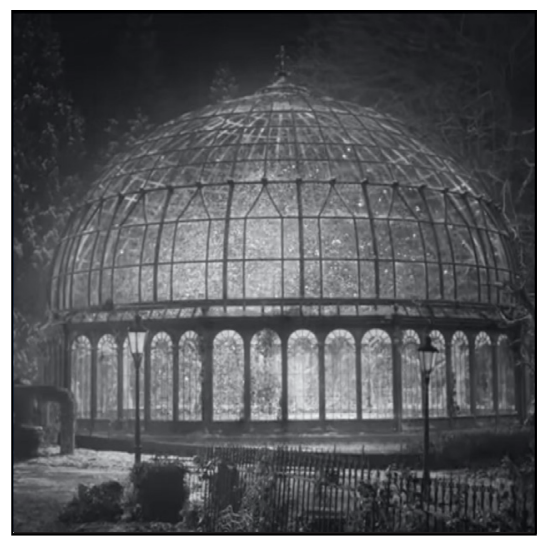

ASCENT

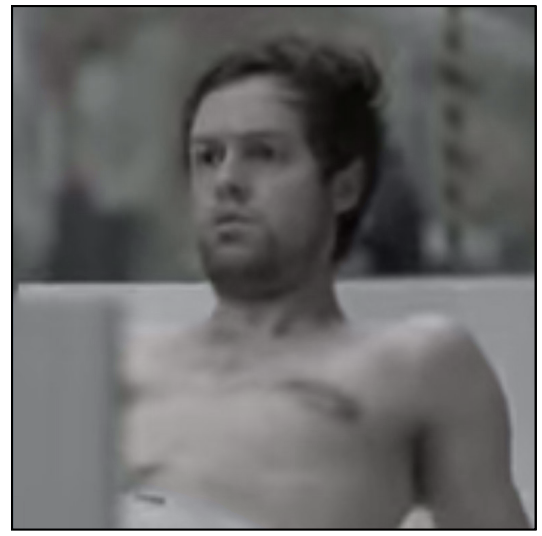

THE CLOSET

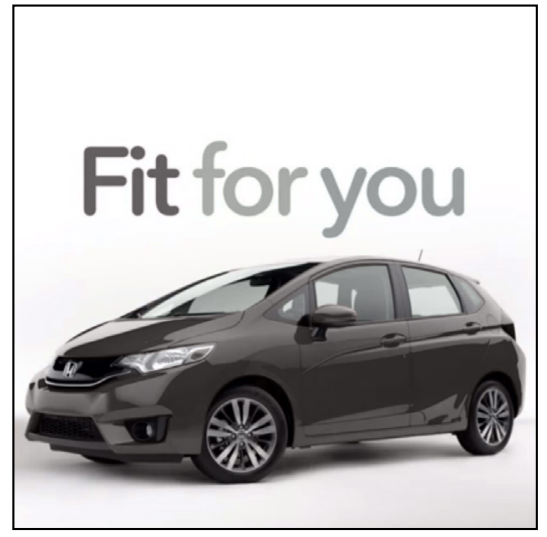

CLOCKS, WRESTLERS, CREEPS AND CENTAURS

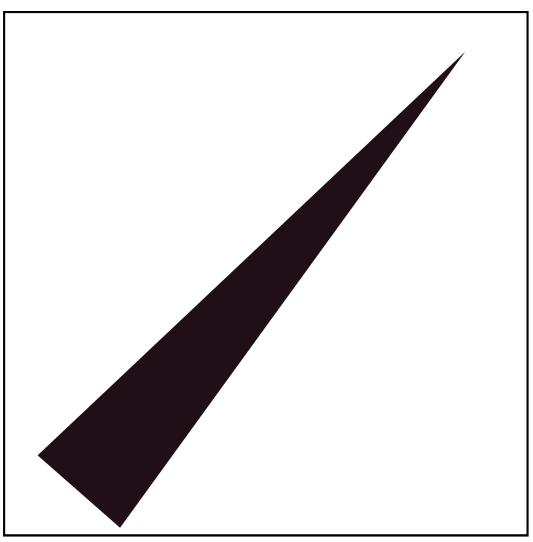

FRAME

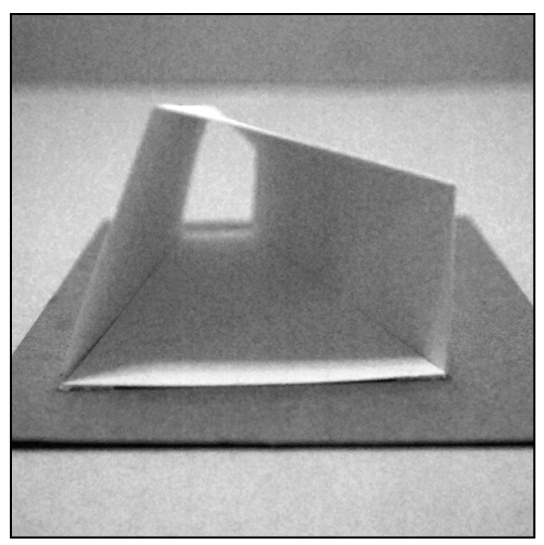

5GUM (2014)

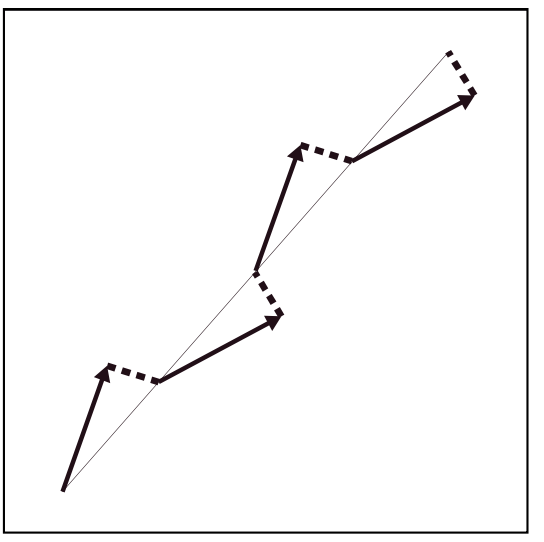

MISDIRECT

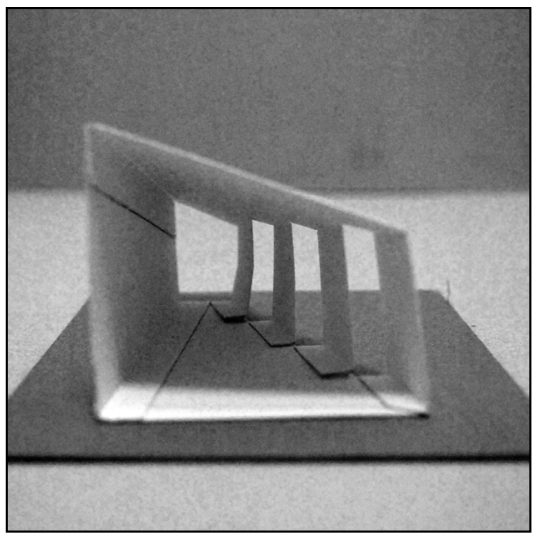

CANAL+ (2009)

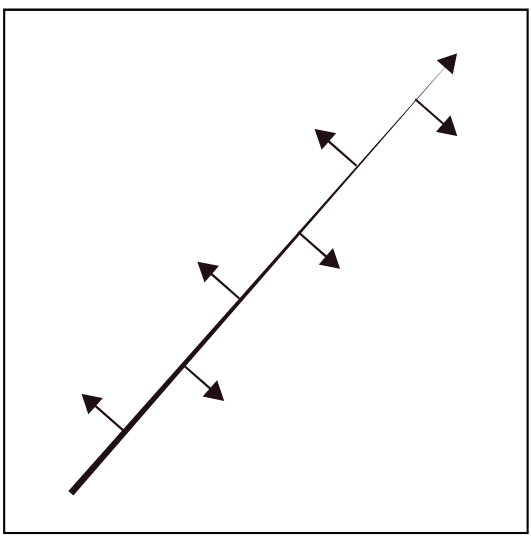

FRAGMENTS

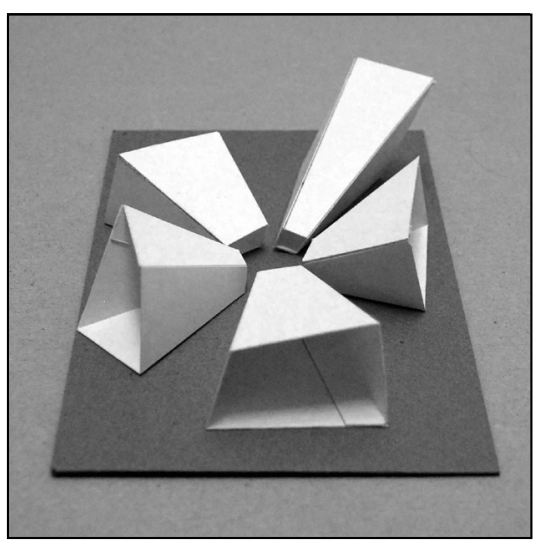

HONDA FIT (2014) 

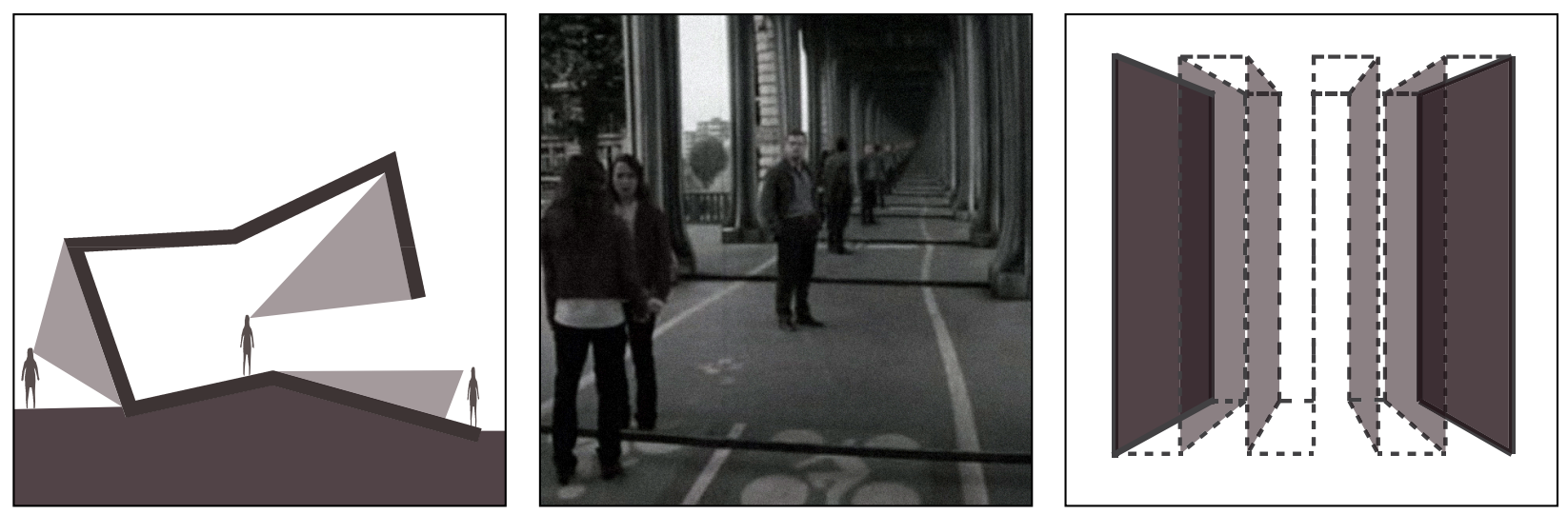

\section{FOLD}

A PART DOUBLED OR LAID OVER ANOTHER
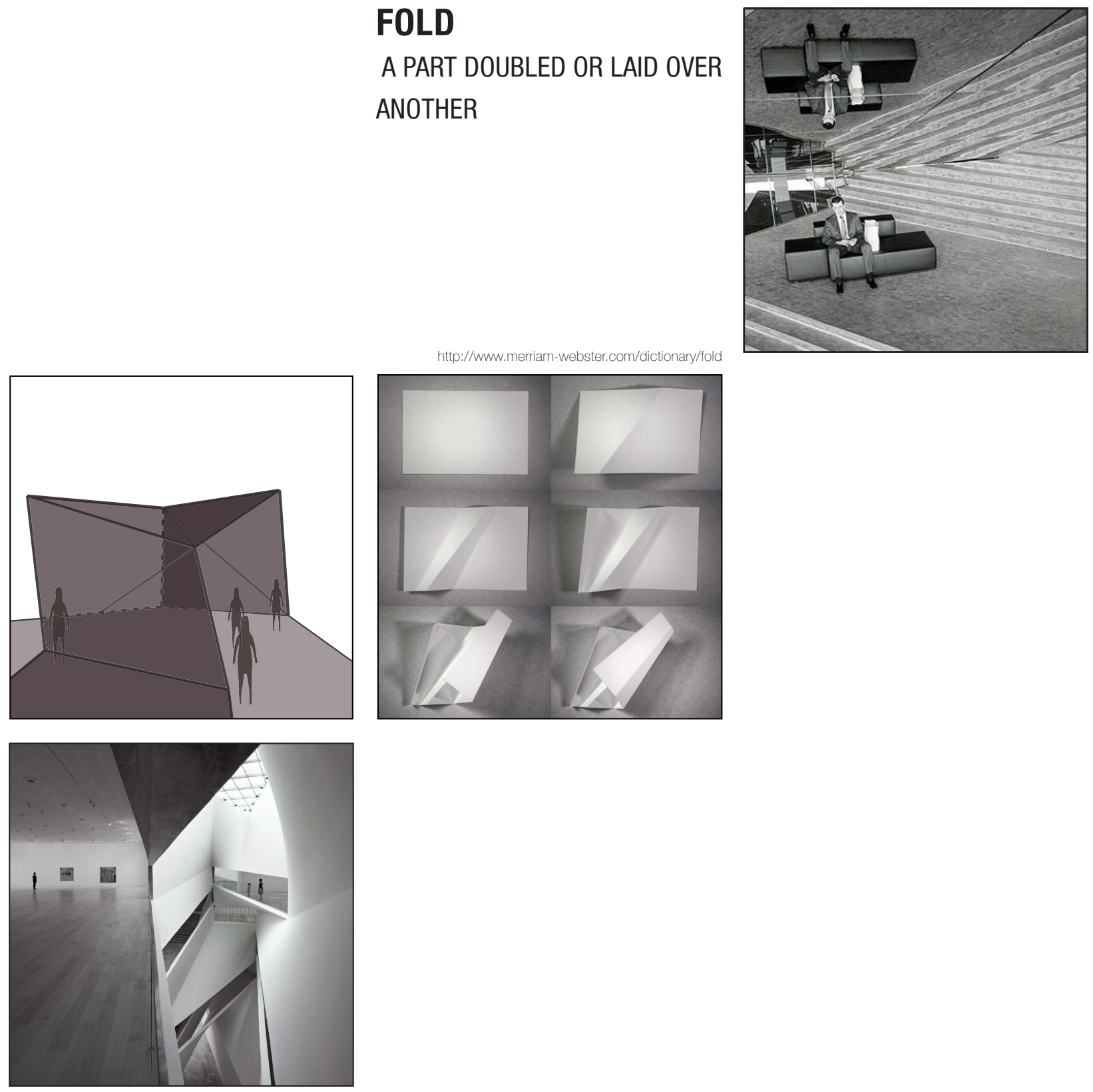

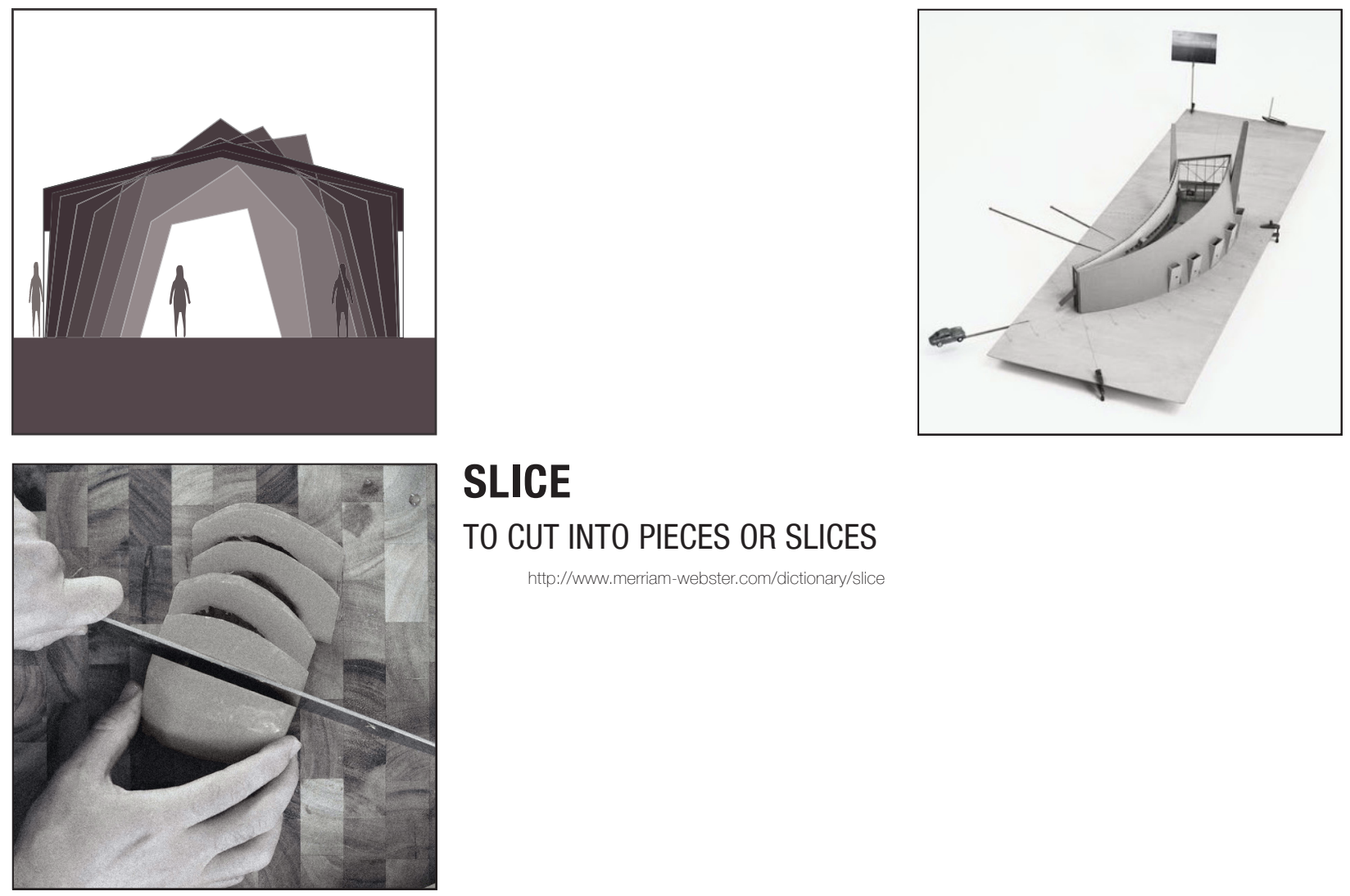

\section{SLICE}

TO CUT INTO PIECES OR SLICES

http://www.merriam-webster.com/dictionary/slice
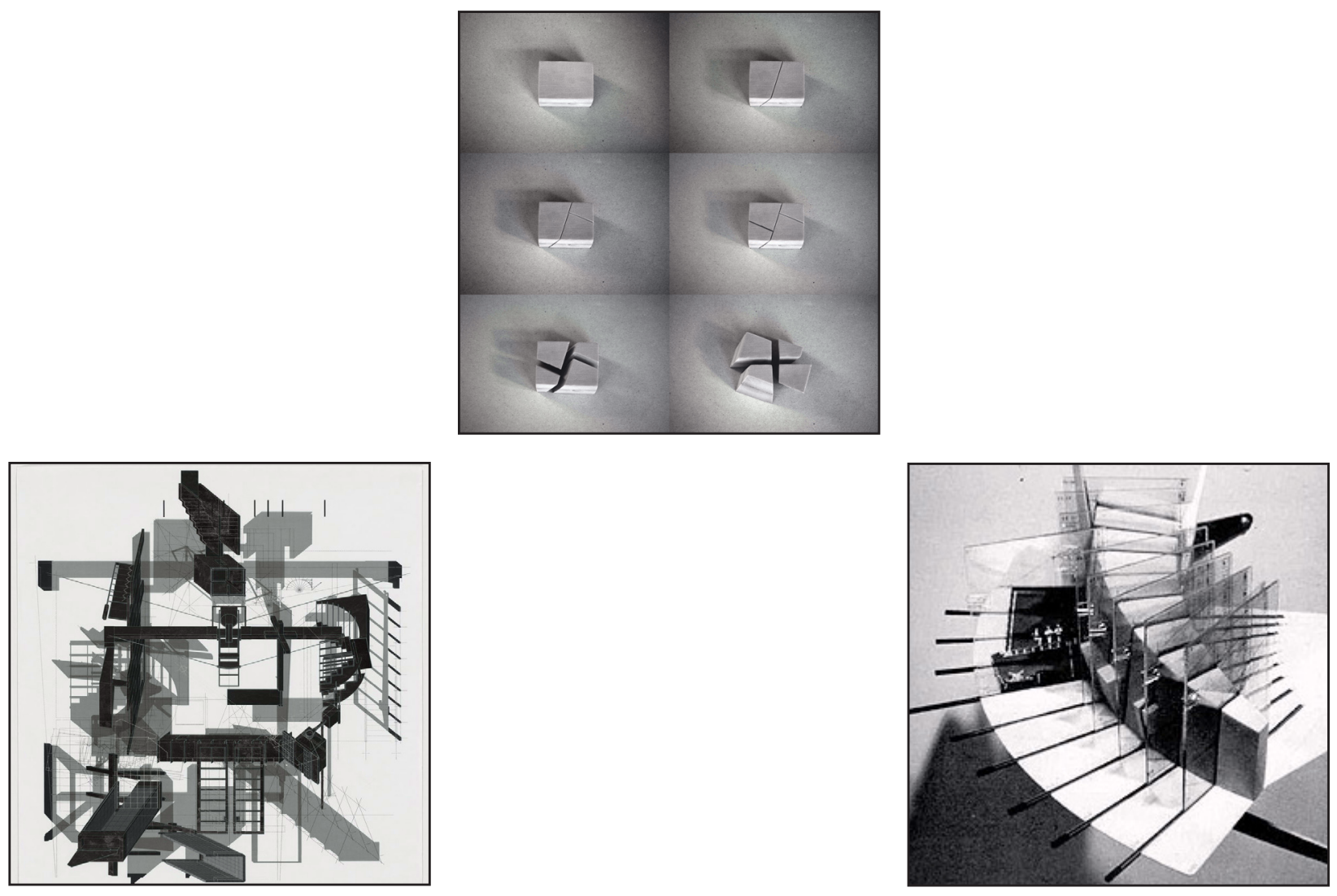

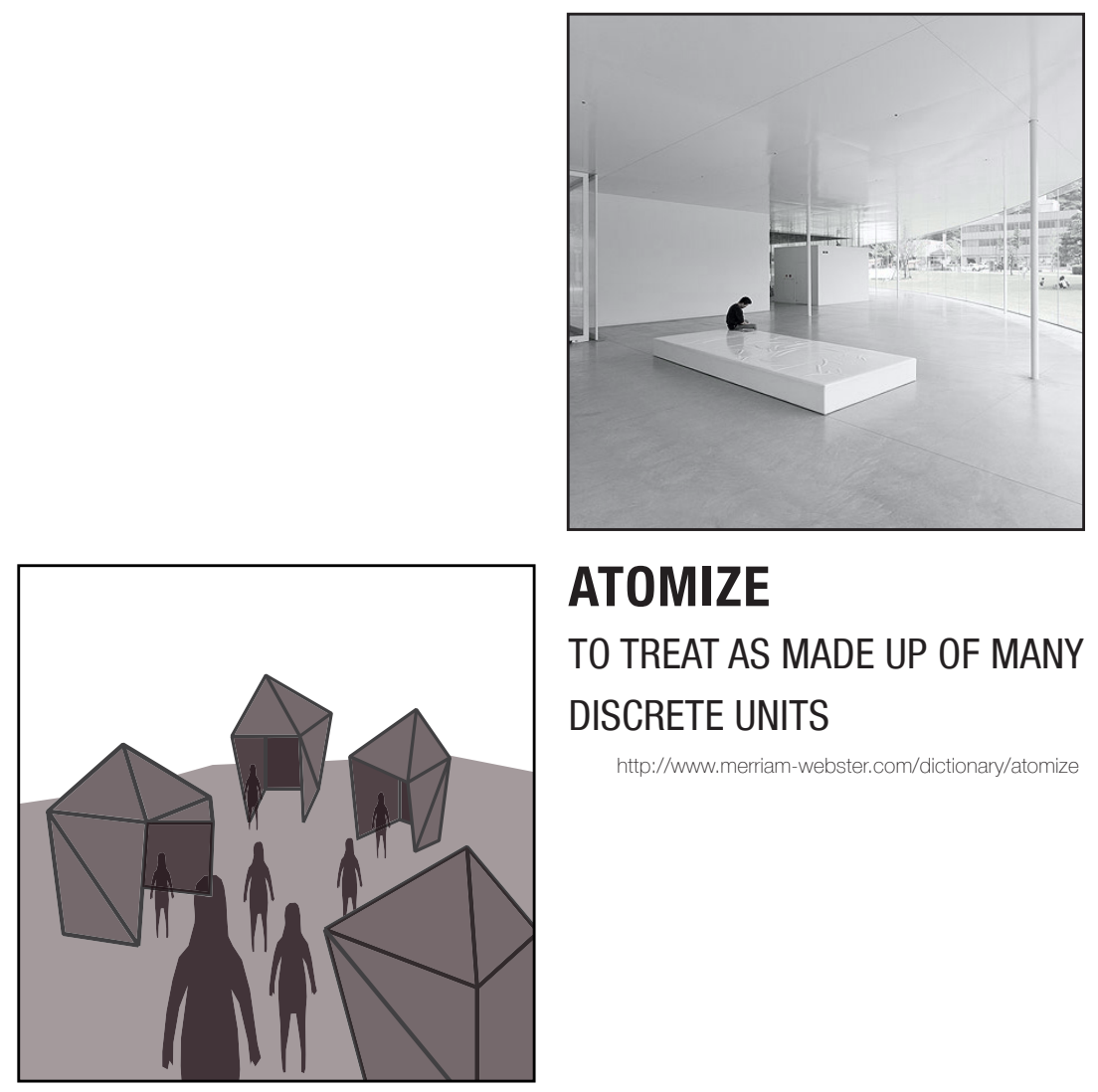

\section{ATOMIZE}

TO TREAT AS MADE UP OF MANY

\section{DISCRETE UNITS}

http://www.merriam-webster.com/dictionary/atomize
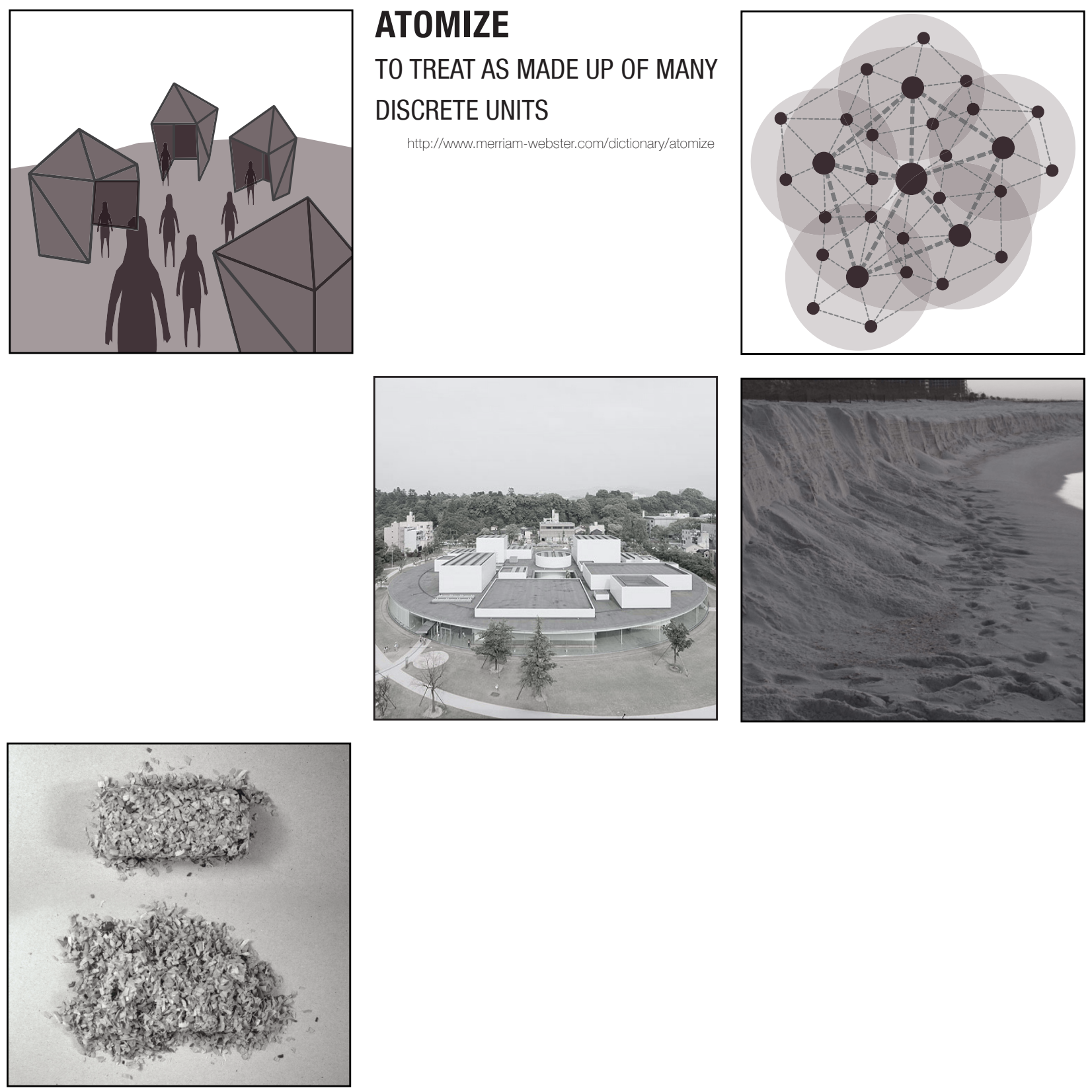


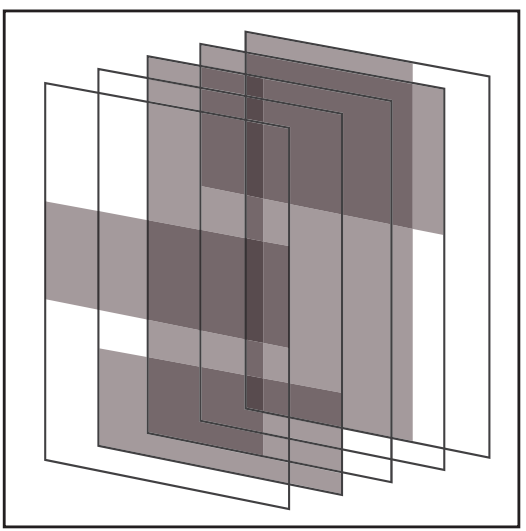

\section{DISTANCE}

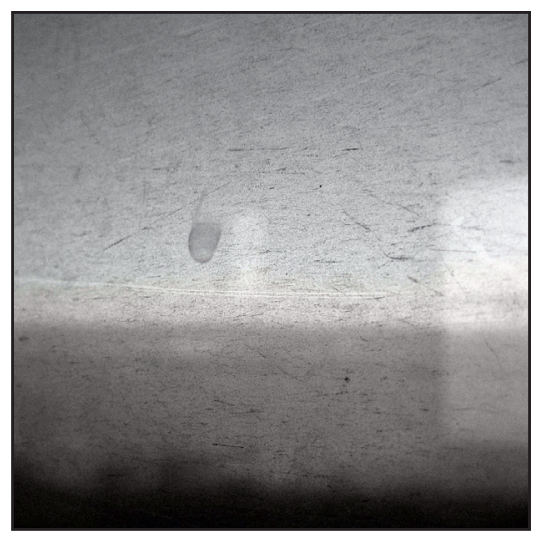

THE DEGREE OR AMOUNT OF

SEPARATION BETWEEN TWO

POINTS, LINES, SURFACES, OR

OBJECTS

http://www.merriam-webster.com/dictionary/distance
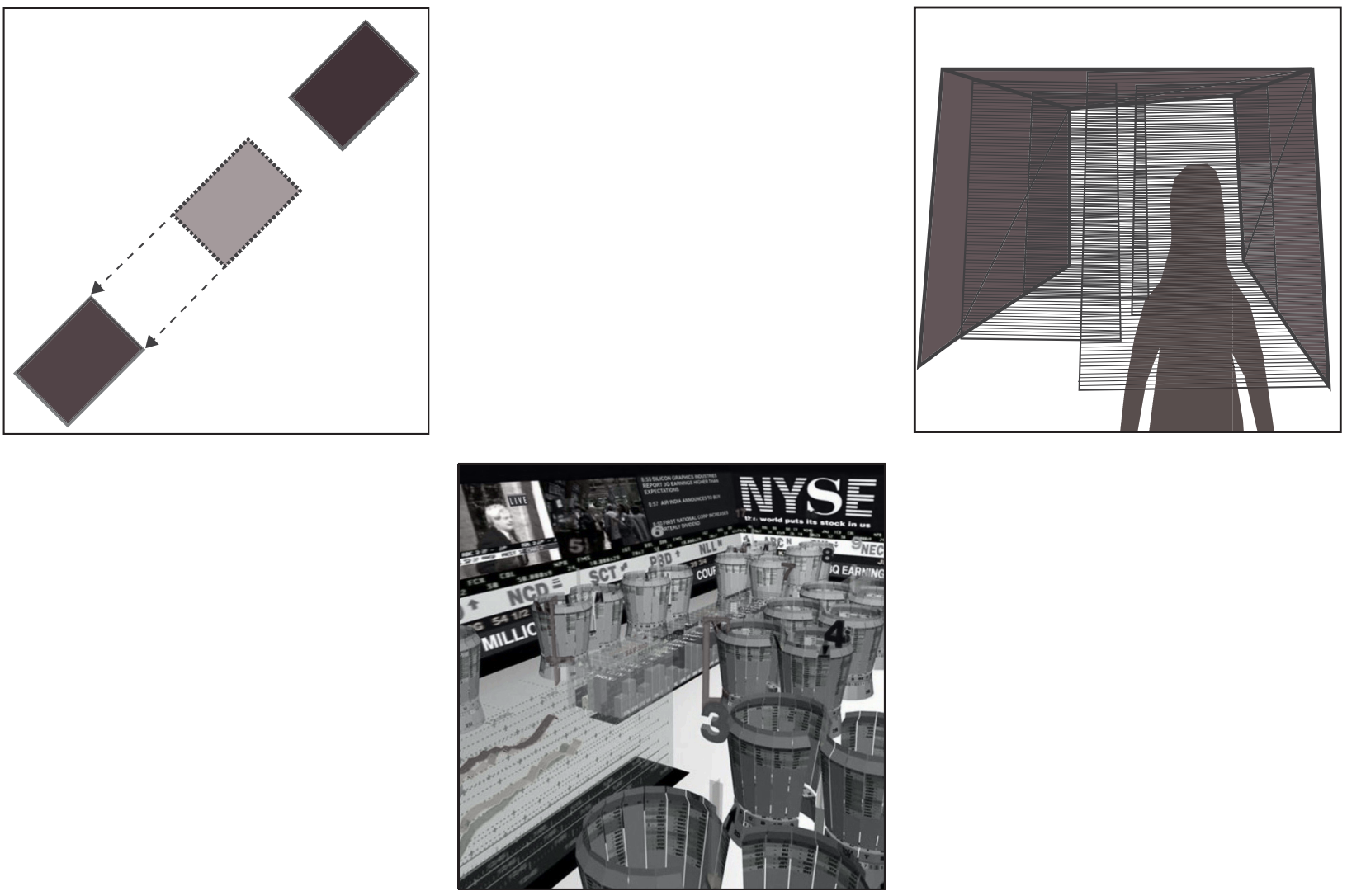
FIGURE 21 EXPERIMENT

Triparte Diagram: Disorient

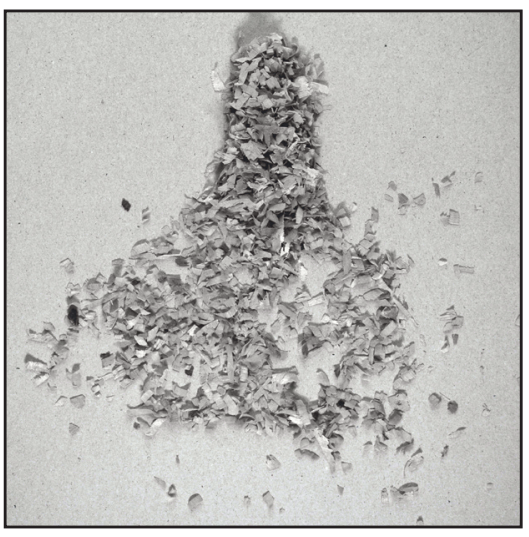

\section{DISORIENT}

TO CAUSE TO LOSE BEARINGS

DISPLACE FROM NORMAL

POSITION OR RELATIONSHIP

http://unw.merriam-webster.com/dictionary/disorient
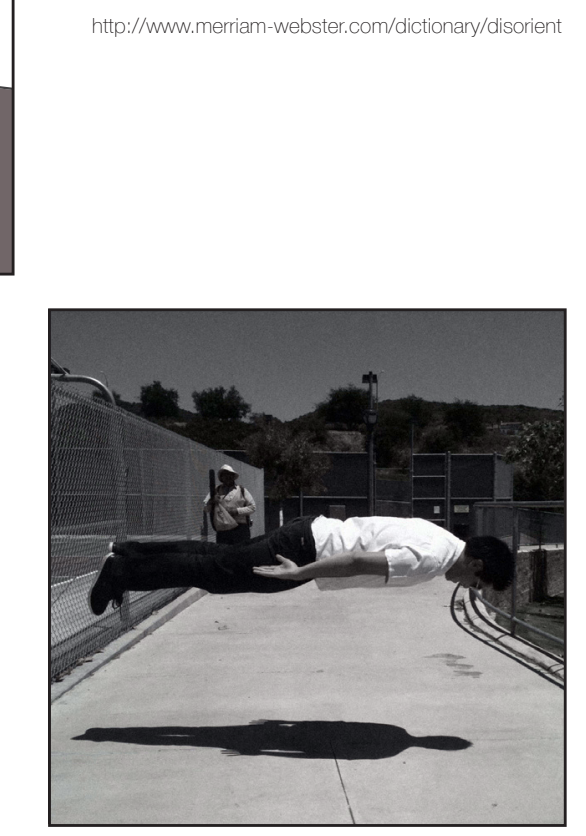

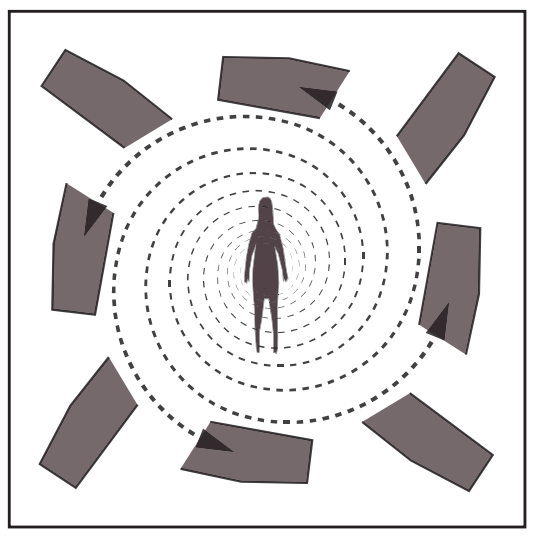
列

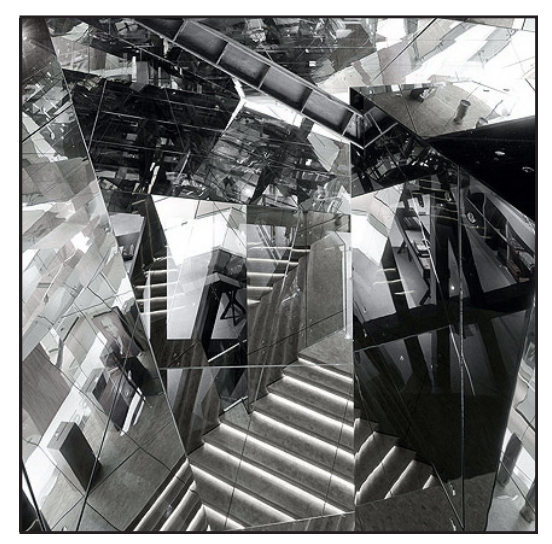




\section{CHAPTER 7}

\section{PRECEDENTS}

\subsection{SPIRAL}

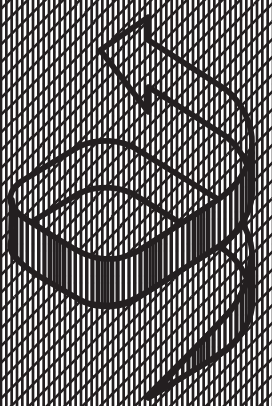

The spiral plan creates a definitive narrative through the structure. The BIG proposal sees each different floor or groupings of floors lead the user through the catalogue of the Axel Springer brand. Ultimately this scheme structures circulation to the periphery and gives a certain degree of visibility in the court yard to connect the spaces.

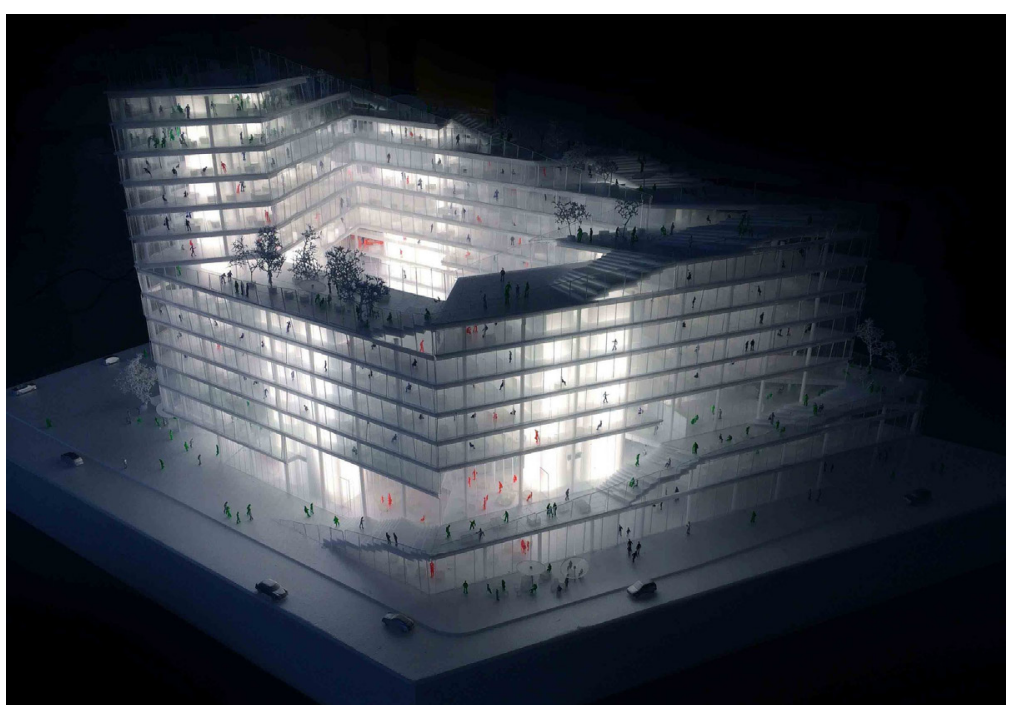

FIGURE 22 PRECEDENT

\section{NEW MEDIA CAMPUS COMPETITION}

Bjarke Ingels Group

Berlin, Germany 


\subsection{PERIMETER}

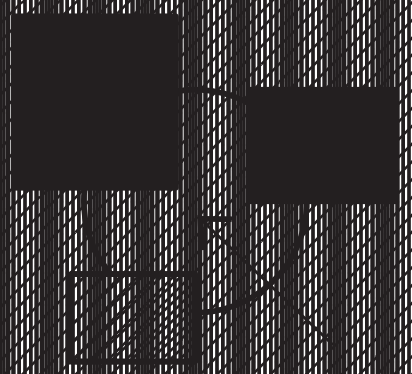

The inherently internal nature of many programmatic elements in media buildings often create highly isolated spaces with minimal connectivity to the other spaces. The focus of TV Azteca Film and TV Sets Campus by at103 and Media Library and Cultural Centre by Barbotin + Gresham is to inject human interaction by structuring the isolated program elements along connective spaces. The perimeter scheme creates a buffer to the outside while centralizing circulation increasing inter-human visibility.

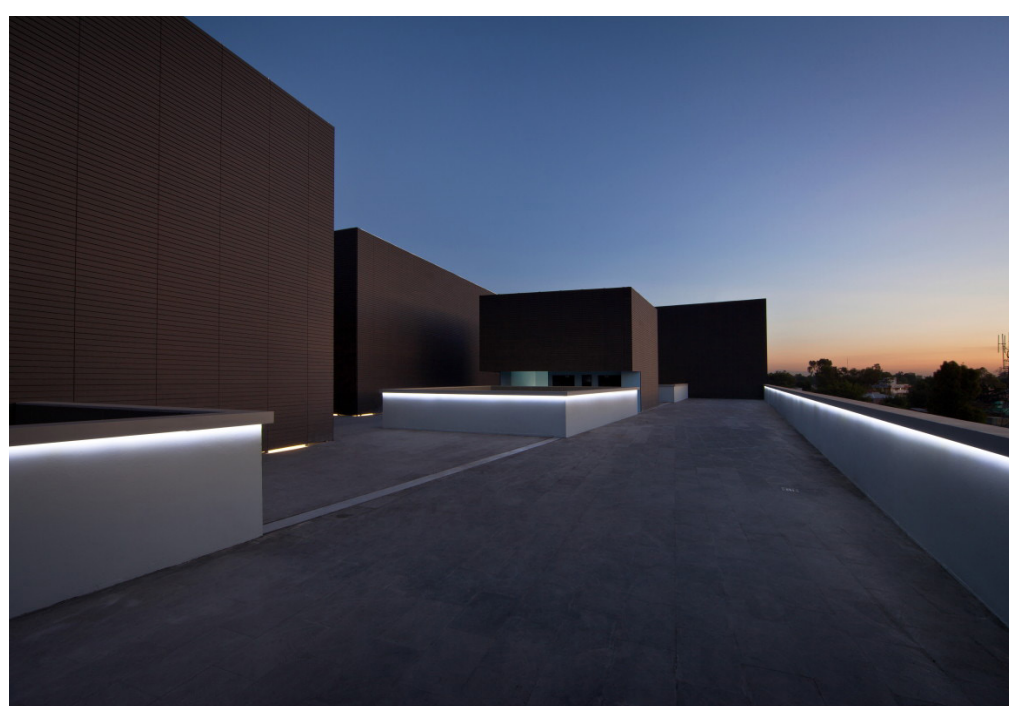

FIGURE 23 PRECEDENT

TV AZTECA FILM \& TV SETS CAMPUS 2012 at103

Tezozomoc, Xico, Mexico $11870 \mathrm{~m}^{2}$
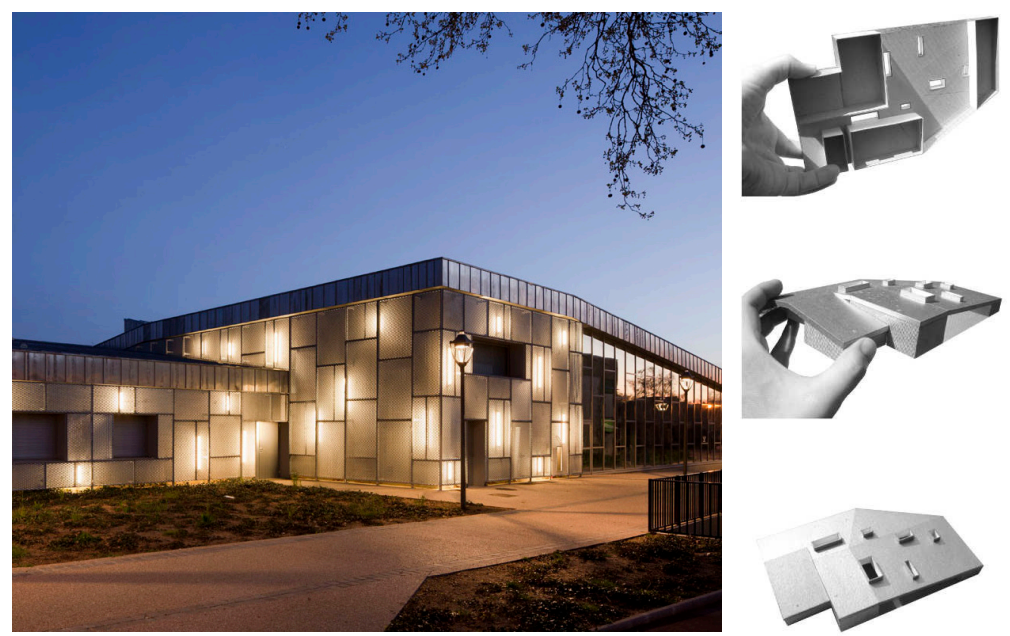

FIGURE 24 PRECEDENT

MEDIA LIBRARY AND CULTURAL CENTRE 2010

Barbotin + Gresham Architects

Lisses, France

$1420 \mathrm{~m}^{2}$ 


\subsection{CLOUD}

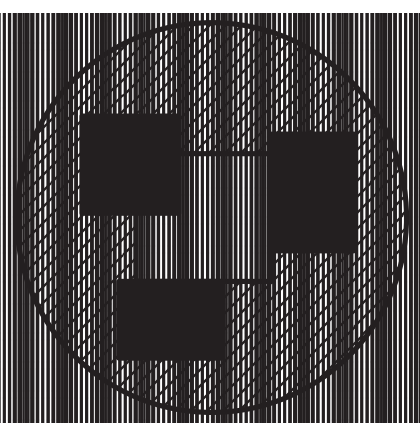

Similar to the perimeter scheme, the Cloud Plan places an emphasis on the creation of a highly visible central core three dimensionally. The OMA and Buro-OS entries for the Axel Springer New Media Campus competition both aim to increase work flow efficiency through a heightened sense of visibility. The large media publishing houses numerous disparate companies under the same brand.

The Cloud scheme programmatically shifts the individual office spaces to the perimeter while at the core exposing the work of individual departments and creating a vibrant social space.

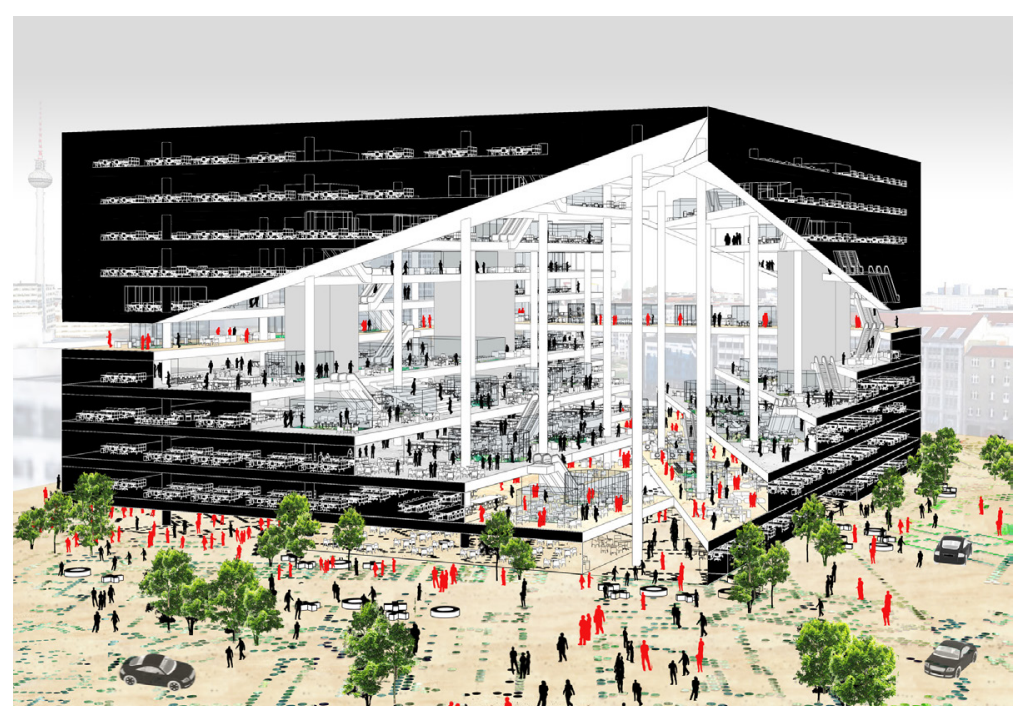

FIGURE 25 PRECEDENT

\section{NEW MEDIA CAMPUS COMPETITION}

OMA

Berlin, Germany
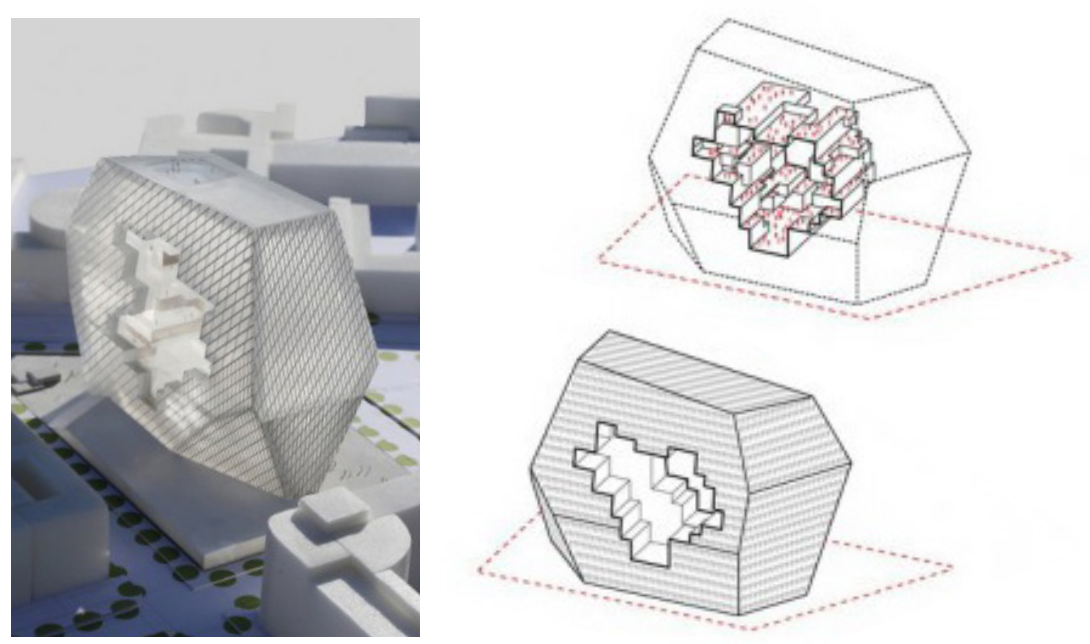

FIGURE 26 PRECEDENT

NEW MEDIA CAMPUS COMPETITION

Buro-OS

Berlin, Germany 


\subsection{EXPOSURE}
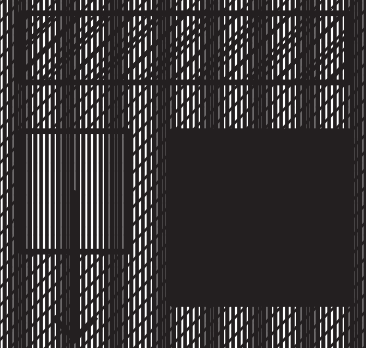

Whenever possible the

NMIT Arts and Media Building by Irving Smith Jack Architects and CBC Headquarters Vancouver by Dialog Design focus on the exposure of process. From the extreme transparency of the NMIT building connecting the campus to the activities inside to the CBC news desk which overlooks a busy street, both projects aim to exhibit the work currently underway. Both projects achieve this exposure with outward facing apertures, and additionally in the $\mathrm{CBC}$ headquarters through the layering of programmatic elements composing a vignette of the gathering of news during the presentation of news.

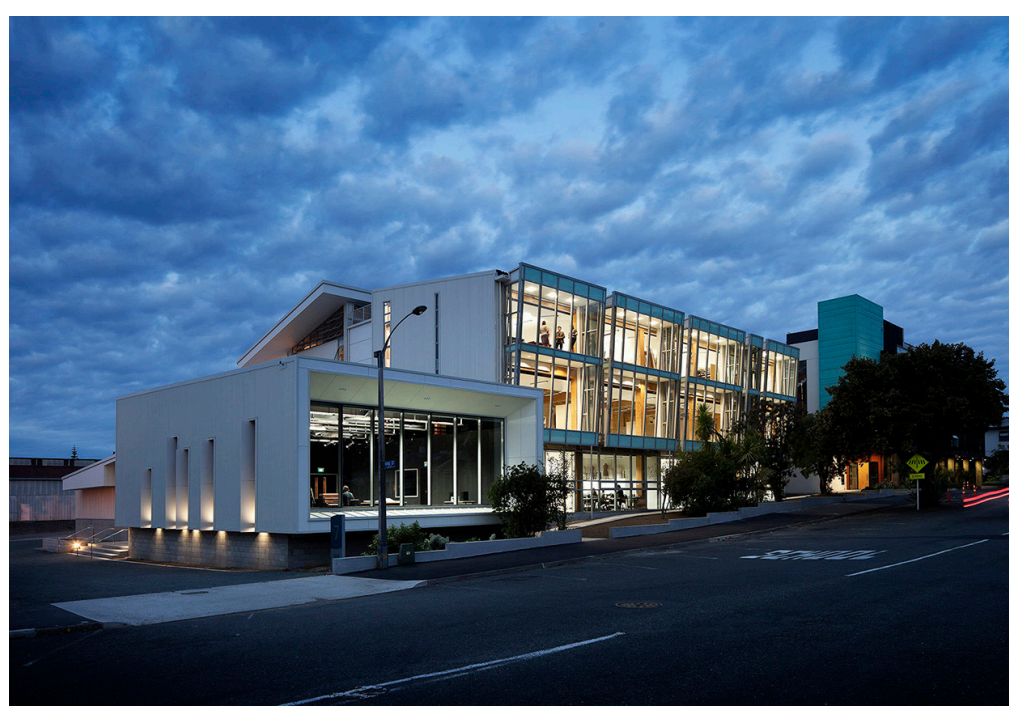

FIGURE 28 PRECEDENT

\section{NMIT ARTS \& MEDIA 2010}

Irving Smith Jack Architects

Nelson, New Zealand

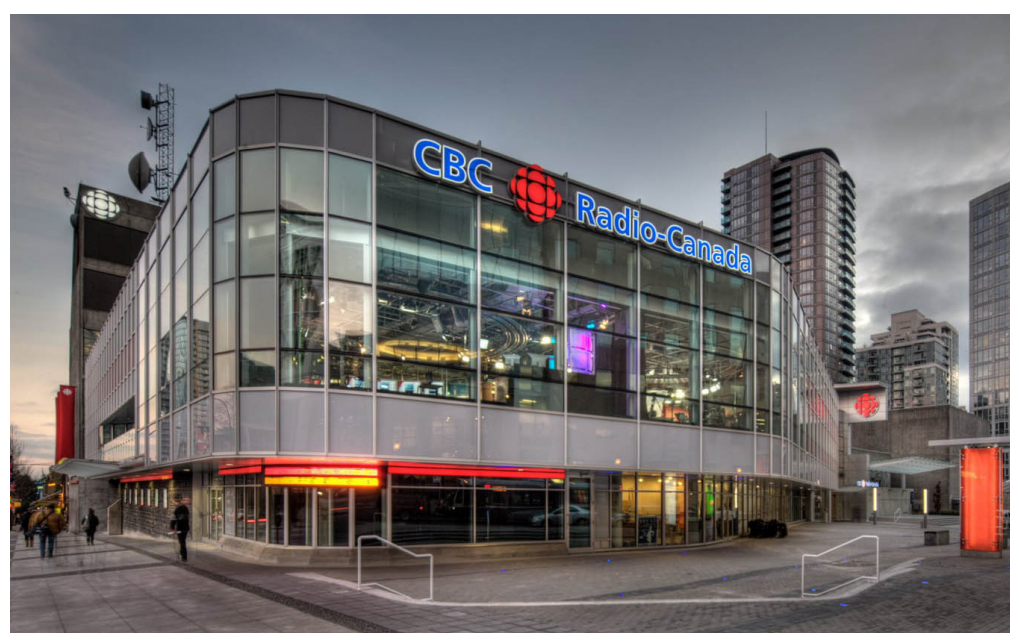

FIGURE 27 PRECEDENT

CBC BROADCASTING CENTRE EXPANSION 2010

DIALOG Design

Vancouver

$31,000 \mathrm{sq} \cdot \mathrm{m}$.

See Appendix 2 for further references (technical drawings). 


\subsection{NARRATIVE}

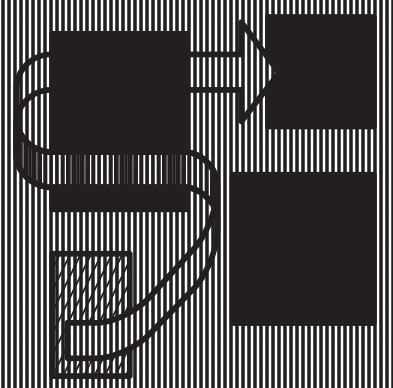

Similar to the spiral

scheme seen in the BIG proposal for the Axel Springler New Media Campus, the narrative scheme of the Columbia College Chicago Media Production Centre by Studio Gang focuses on the development of a narrative through circulation and nodes. As users makes their way through the facility they are exposed to a series of post-production programming. Each node adds a layer of augmentation to the process, emphasizing a particular stage in the development of film.

The narrative begins with the presentation of a film, moves through the sound mixing and post production spaces and ends with the raw input of the filming in the sound stage.
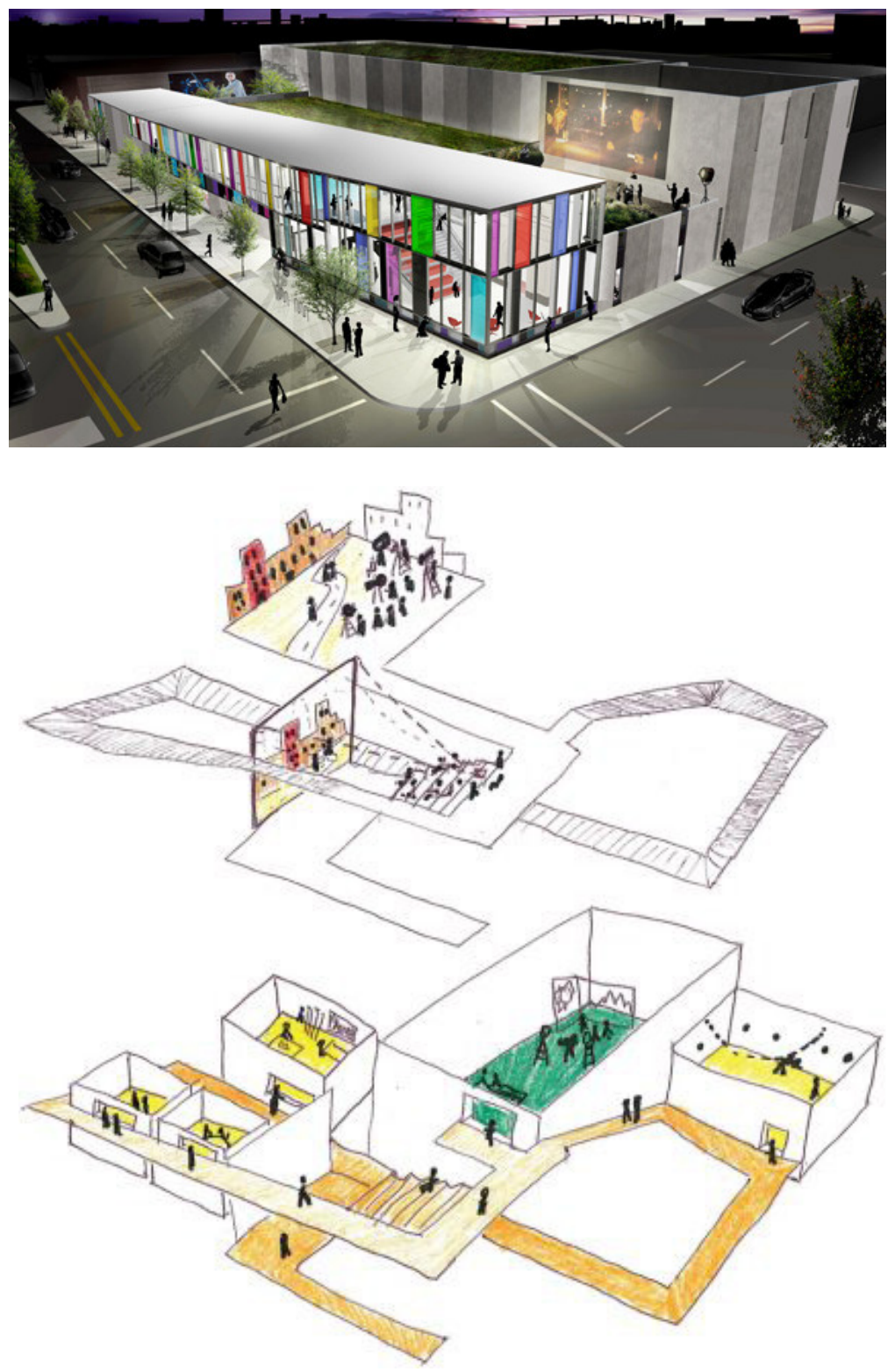

FIGURE 29 PRECEDENT

COLUMBIA COLLEGE CHICAGO MEDIA PRODUCTION CENTER 2010

Studio Gang Architects

Chicago, USA

$3300 \mathrm{~m}^{2}$ 


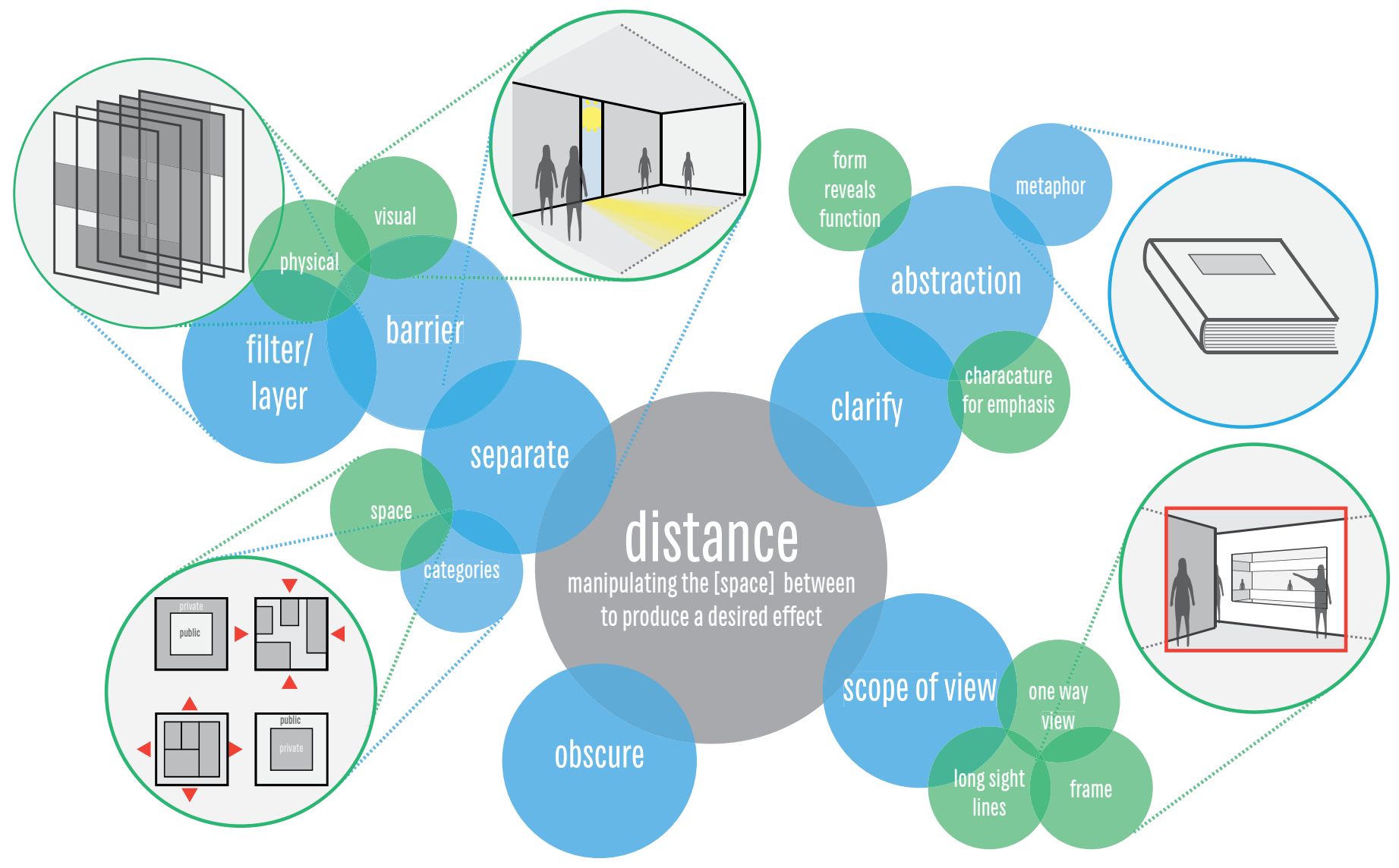

FIGURE 30 DIAGRAM

Conceptual Map: Distance 


\section{CHAPTER 8}

\section{CONCEPTUAL MAP}

The conceptual maps highlight key aspects derived from spatial and textual research. The three key themes of distance, disorientation and exposure are exploded into individual facets and explored diagrammatically.

\subsection{DISTANCE}

Distance is primarily understood as the manipulation of space between two objects. It can be used to clarify or increase the understanding of an artifact as is witnessed in abstraction and through the use of metaphor. Architecturally the use of distance as a tool for clarity can be utilized when form reveals function and through the overt stylization of elements to emphasize a particular point. Simultaneously distance can be used to obscure an interpretation by removing the original reference points and re-situating the work. Distancing can also be interpreted as the scope of view where a particular view can be framed in a manner which highlights multiple facets as evident in Preston Scott Cohen's Tel Aviv Museum of Art where portals expose and link various exhibits to one another. The overlapping of information creates distance between the works while visually connecting each exhibit. Distance is additionally physically understood through separation and the use of visual and physical barriers to filter the perspectival field of an artifact. 


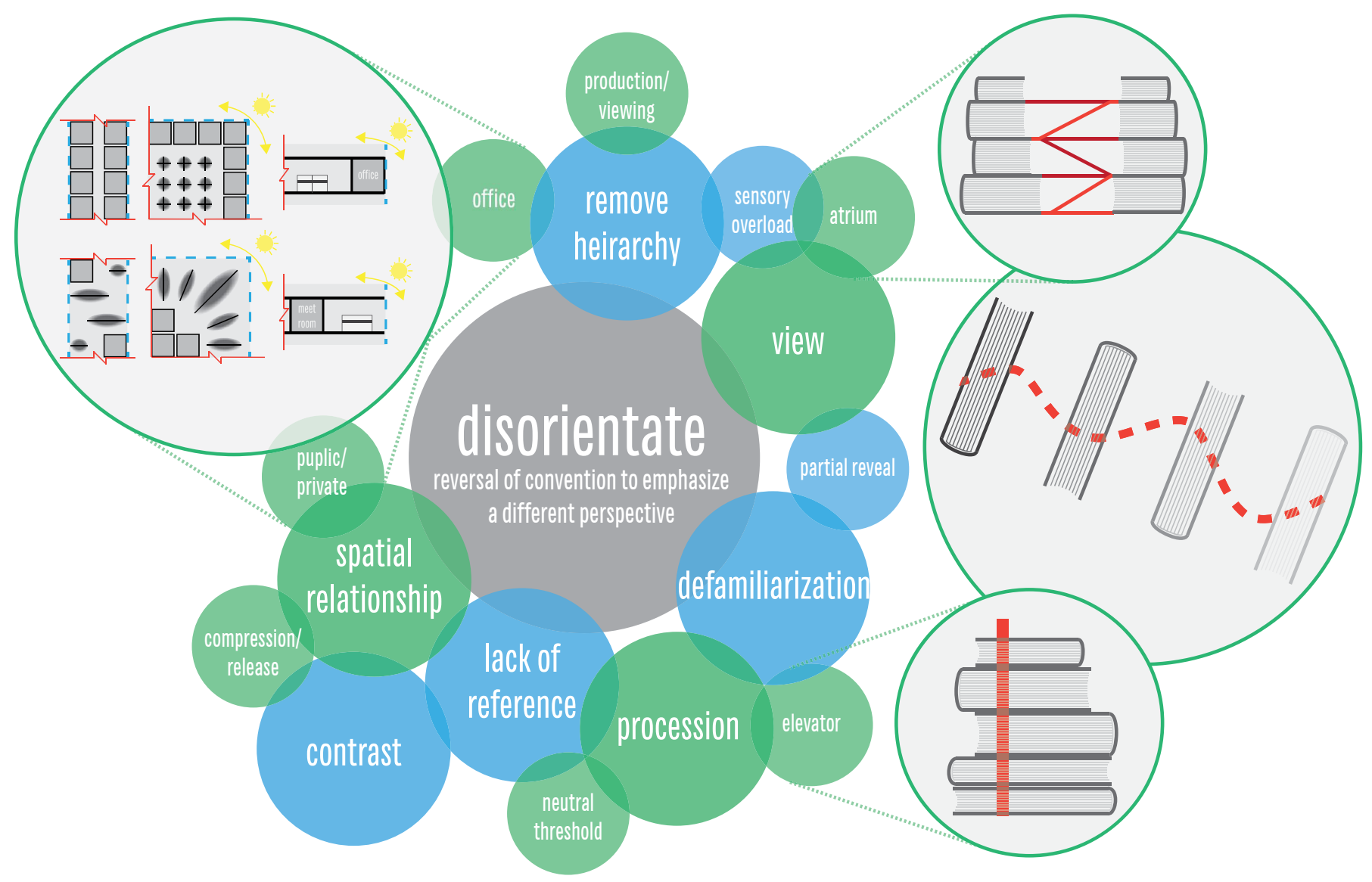

FIGURE 31 DIAGRAM

Conceptual Map: Disorientate

\subsection{DISORIENTATION}

Disorientation utilizes the reversal of convention to emphasize new or previously suppressed facets of a work. Disorientation appears in the removal of hierarchy such as in conditions where a sensory overload is experienced. The atrium becomes a model expressing said condition where the sheer quantity of stimuli disorientates the viewer. Through the removal of hierarchy in well established typologies such as the office building disorientation can be positively attributed to the creation of communal work places where information more fluidly transfers between users. Finally in the lack of reference or defamiliarization of users, disorientation is used to separate the user from space. The elevator for example possesses the ability to radically alter the users understanding of a building. An elevator is a portal between narratives, completely isolating users' and thrusting them into a new and different space at the press of a button. This allows the contrast between spaces to become more dynamic and exemplary of particular conditions. 


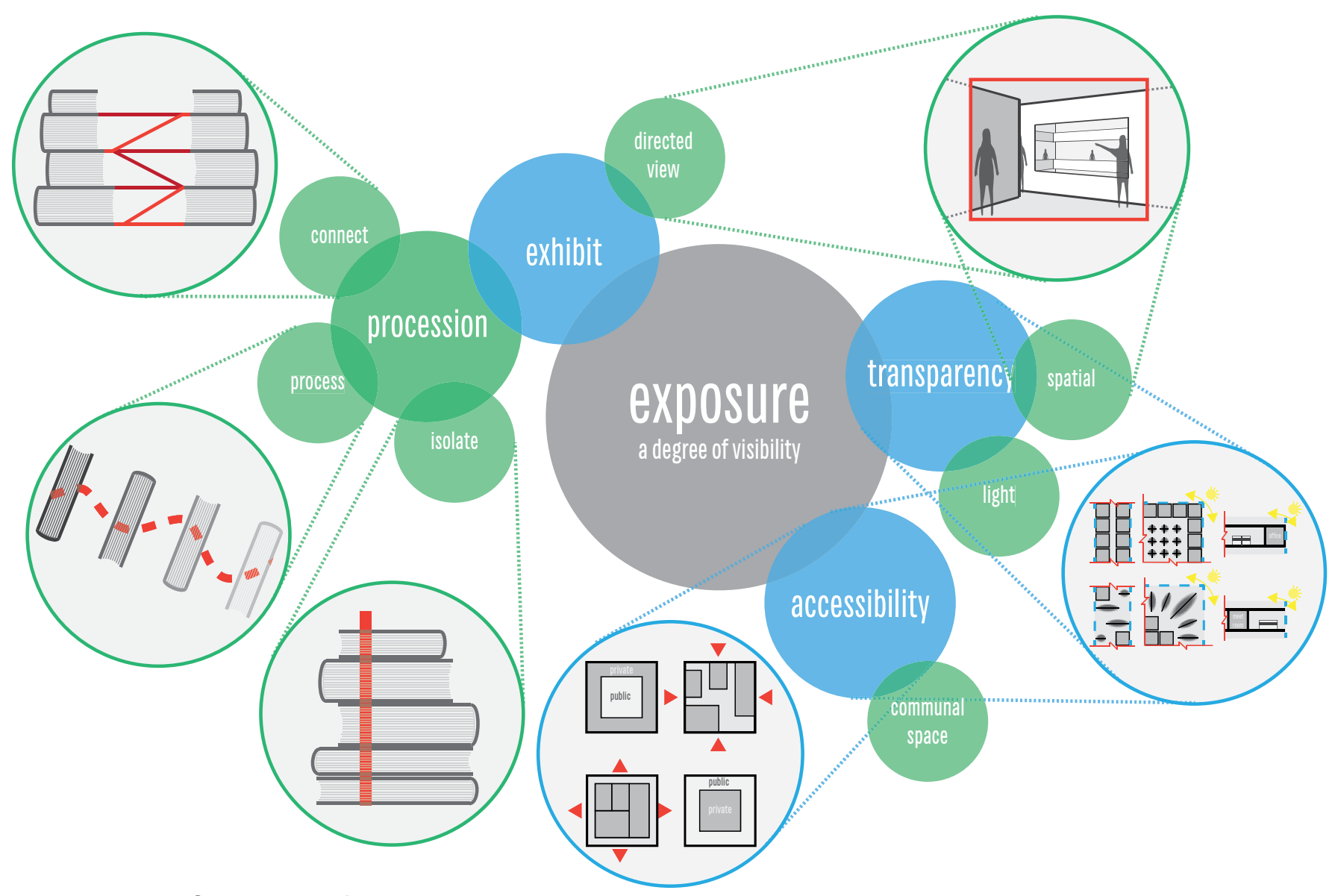

FIGURE 32 DIAGRAM

Conceptual Map: Exposure

\subsection{EXPOSURE}

Exposure can fundamentally be understood as the overall degree of visibility experienced towards an artifact. The definition encompasses both high and low degrees of artifact accessibility. Of particular importance in this thesis is exhibition and how exposure begins to unravel artifacts to expose facets that are not readily experienced. Procession and exhibition allows the user to connect (atrium conditions unite and superimpose facets of the work upon one another), isolate (elevator conditions remove a spatial connection and create disparate spaces) and experience process (deconstructive narrative draw a user through a series of nodes which explain particular faces). 

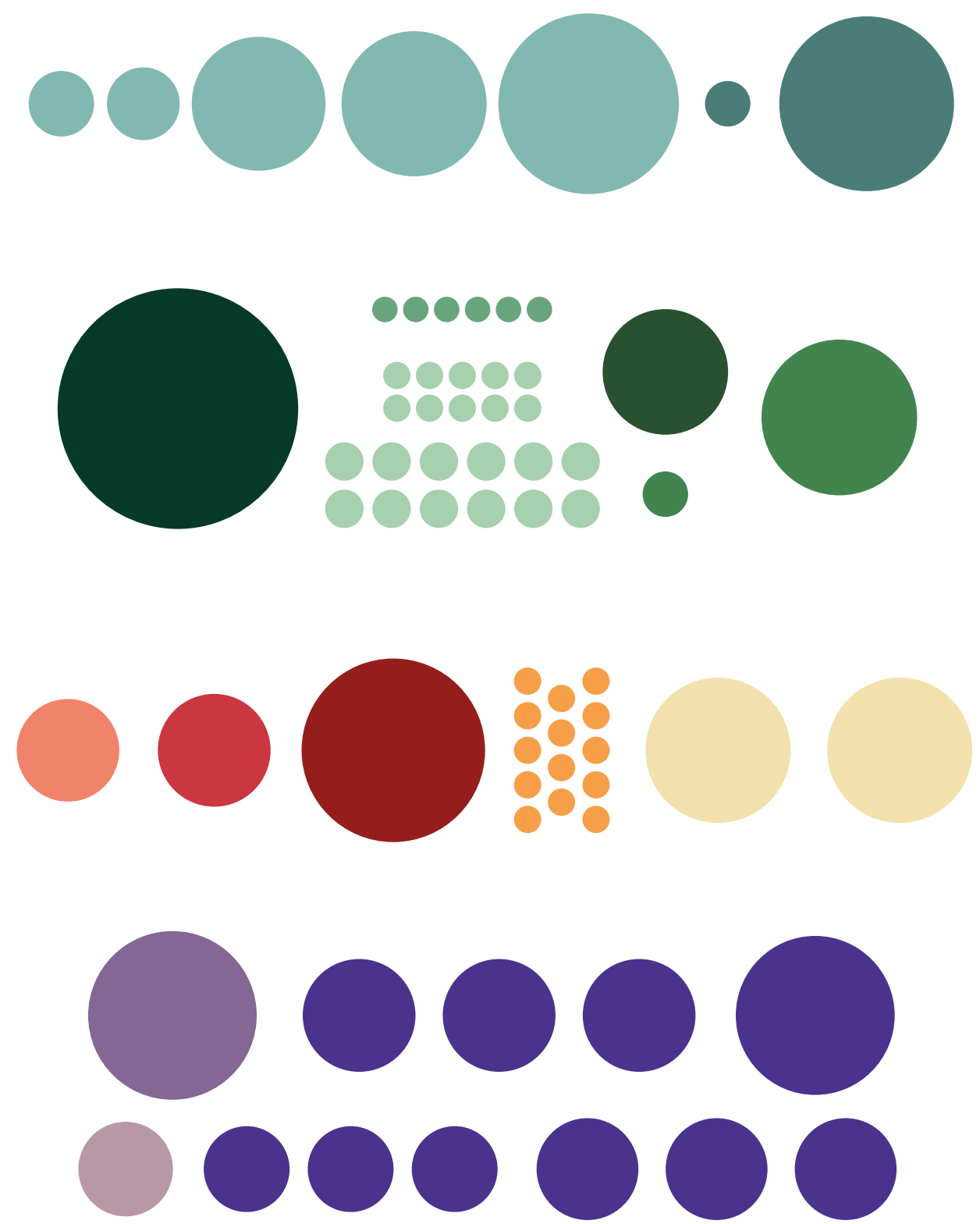

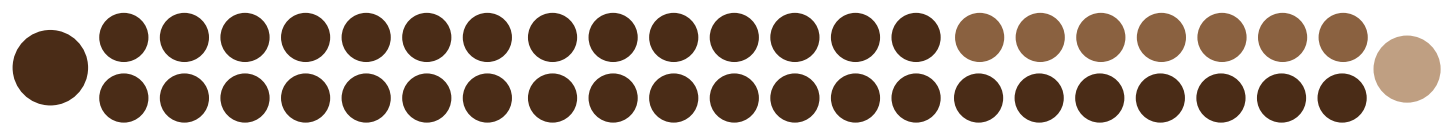

FIGURE 33 DIAGRAM

Programming Analysis: Raw to Final Product 


\section{CHAPTER 9}

\section{PROGRAMMATIC EXPOSURE}

Initially the program has been constructed using precedents and the existing Ryerson Image Arts building as a basis. Each space has been abstracted and colour coordinated using the site as the general reference point. Using the theme of exposure the program was analyzed to develop associations between the spaces, allowing each space to draw and build upon its surrounding relationships.

The first iteration sees the general spatial relationships drawn out to show the connection between education and process spaces, moving towards more final presentation spaces on the right hand side. The second iteration begins to use the general site to cluster the spaces into a series of floors. At this point the primitive semblance to the circulatory narrative developed in future iterations appear. Each cluster begins to emphasize the circular nature of design from exhibition, the creation of raw content, post production, finishing and again with exhibition.

The final iteration creates an experiential section through the building drawing how circulation impacts the clustering of spaces. Circulation has been abstracted to a straightened path through the building and again in the elevator core symbolized by the vertical gray bands.

EXHIBITION SPACE

EXHIBITION ARCHIVE

SOUND STAGE

B/W FILM PROCESSING

NEW MEDIA LAB

MIXING/ RECORDING STUDIO

DIGITAL EDITING SUITE

PHOTOGRAPHY STUDIO

COMPUTER LAB

FIGURE 34 LEGEND

Programing and Scale
IMAGE PROCESSING CENTRE

FILM LOADING ROOM

DARK ROOM

MULTIPURPOSE CLASSROOMS

EQUIPTMENT ROOM

WORKSHOP

FACULTY OFFICE

ADMIN OFFICE

STUDENT OFFICE

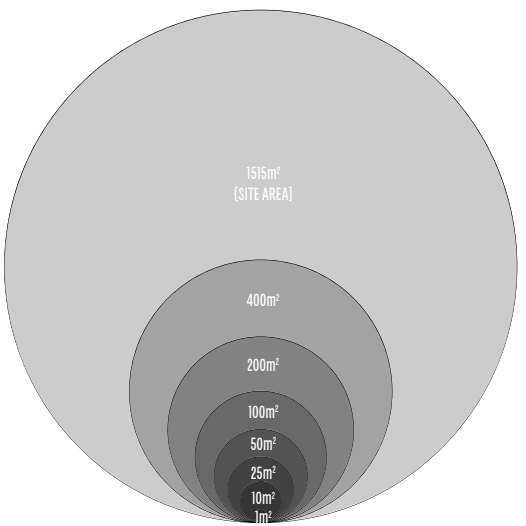

PROGRAMMATIC EXPOSURE 


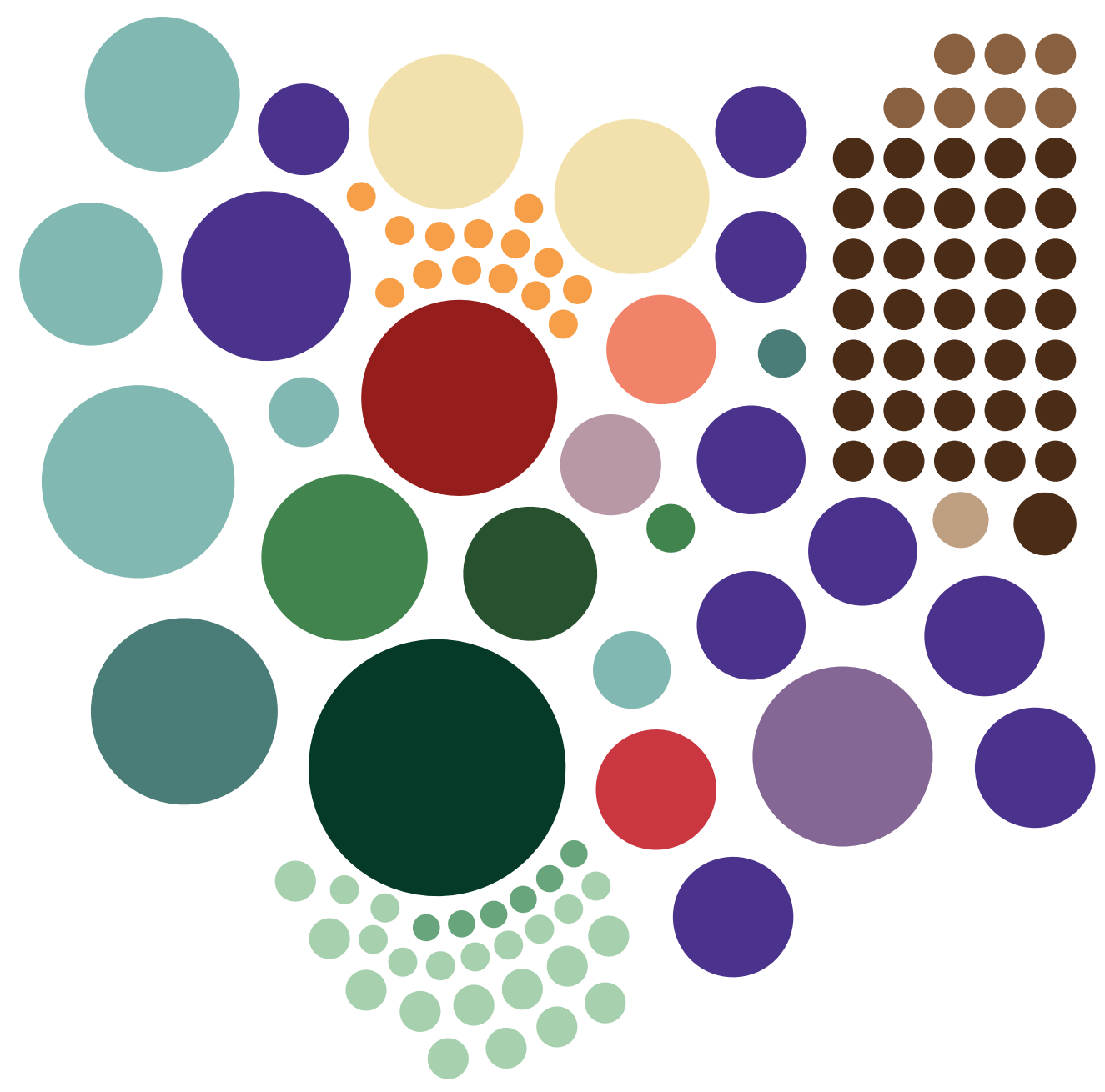

FIGURE 35 DIAGRAM

Programming Analysis: Spacial Relationship 

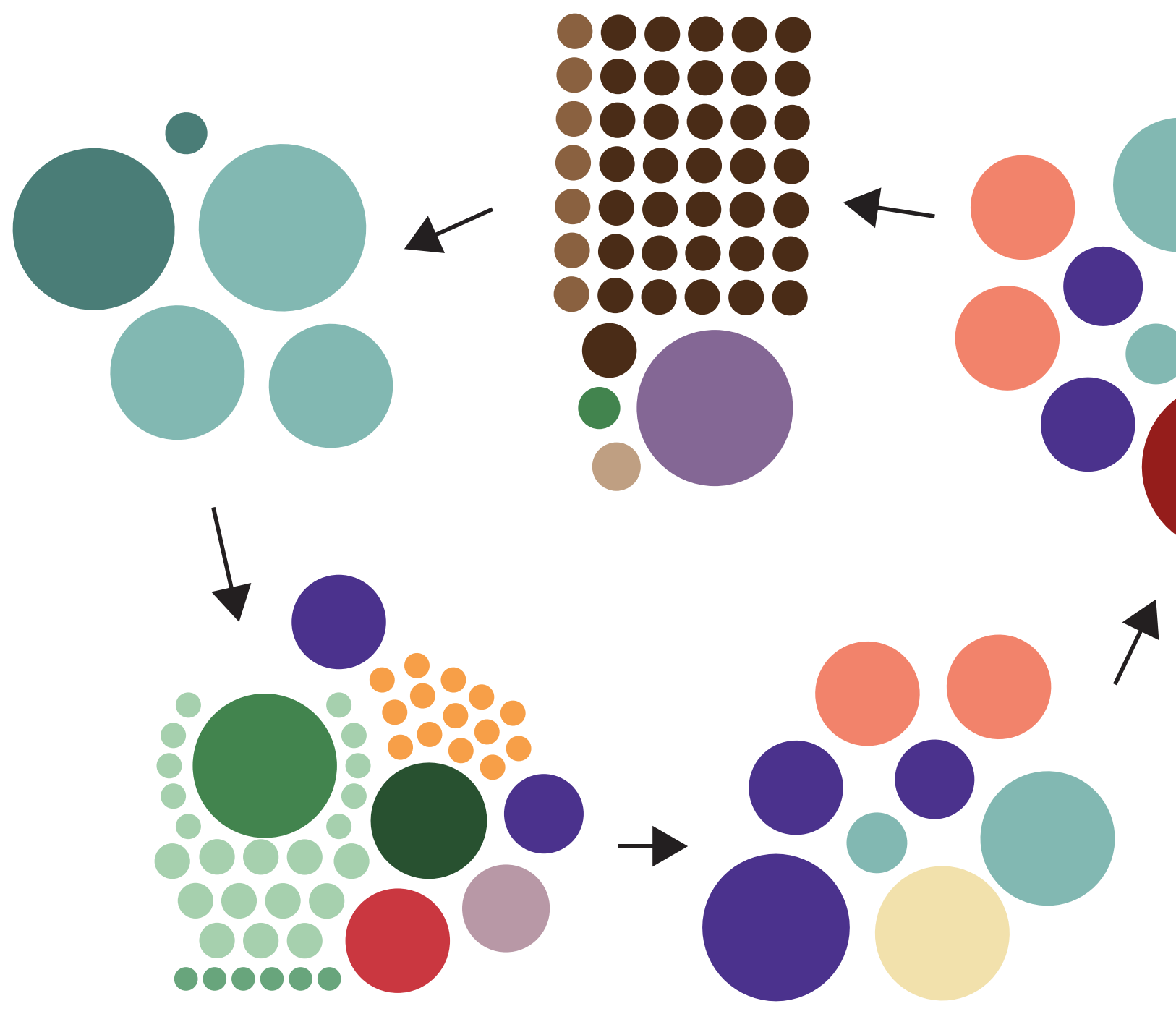

FIGURE 36 DIAGRAM

Programming Relationship: Interdisciplinary Clustering 


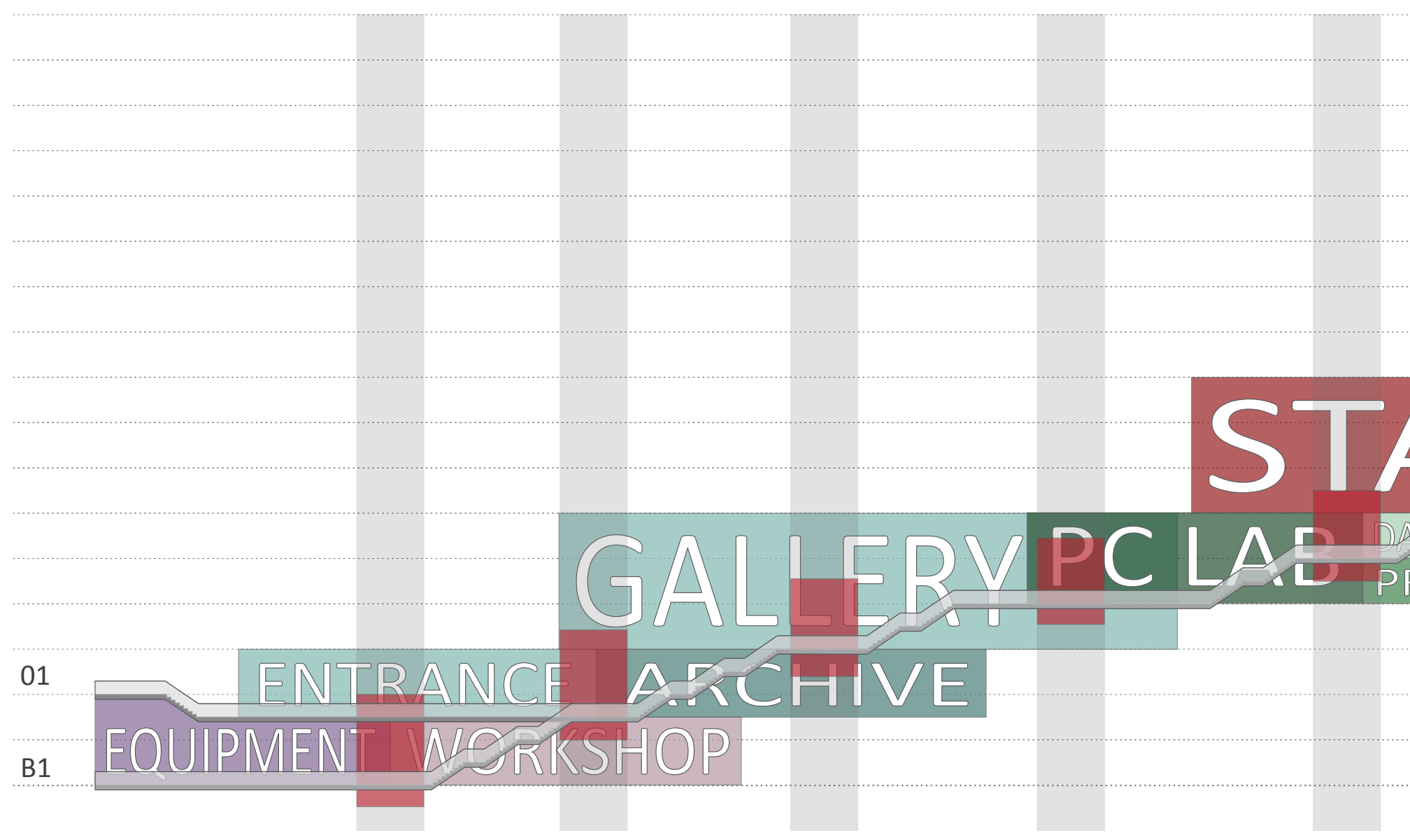

FIGURE 37 DIAGRAM

Programming Analysis, Sectional Relationship 


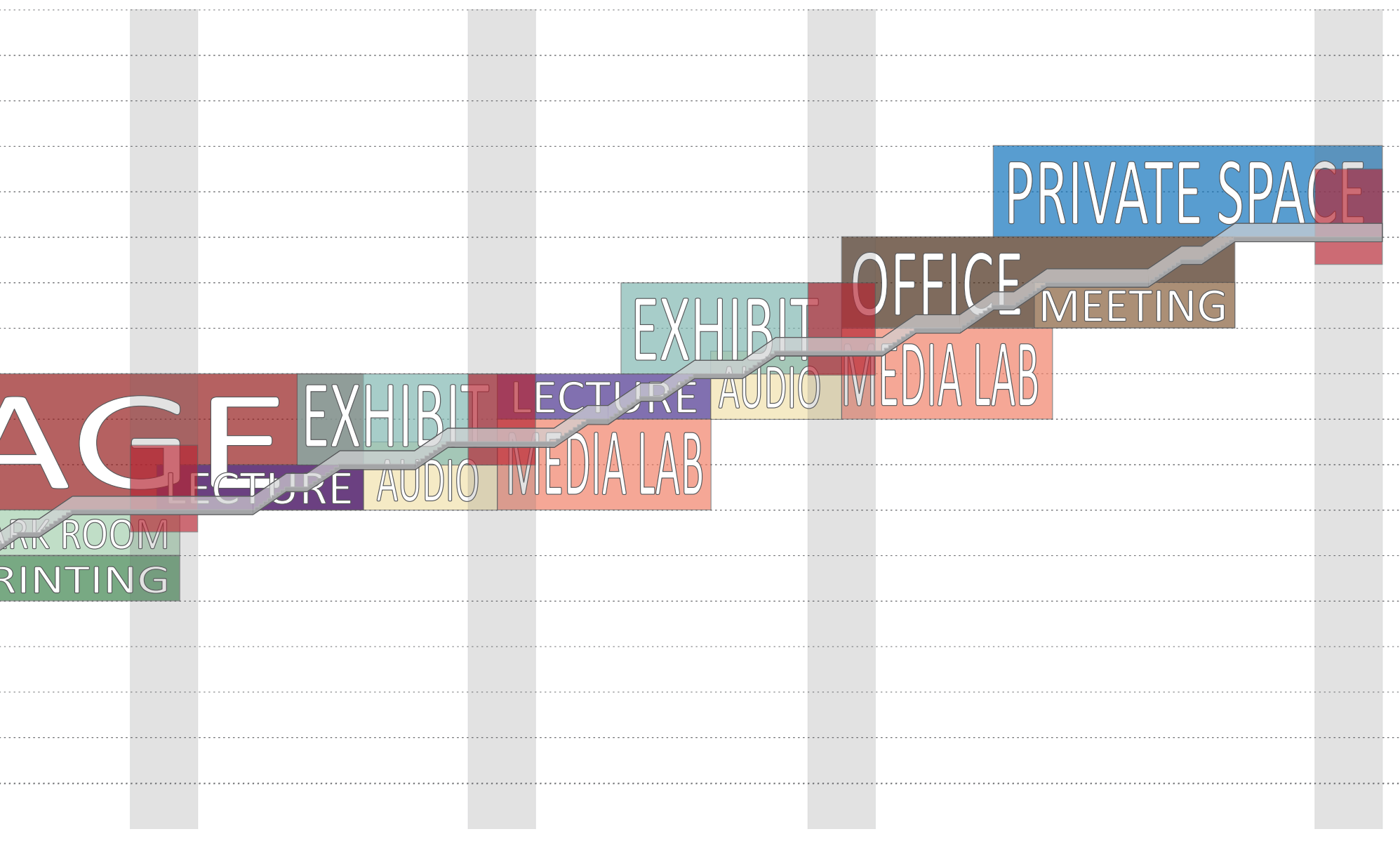




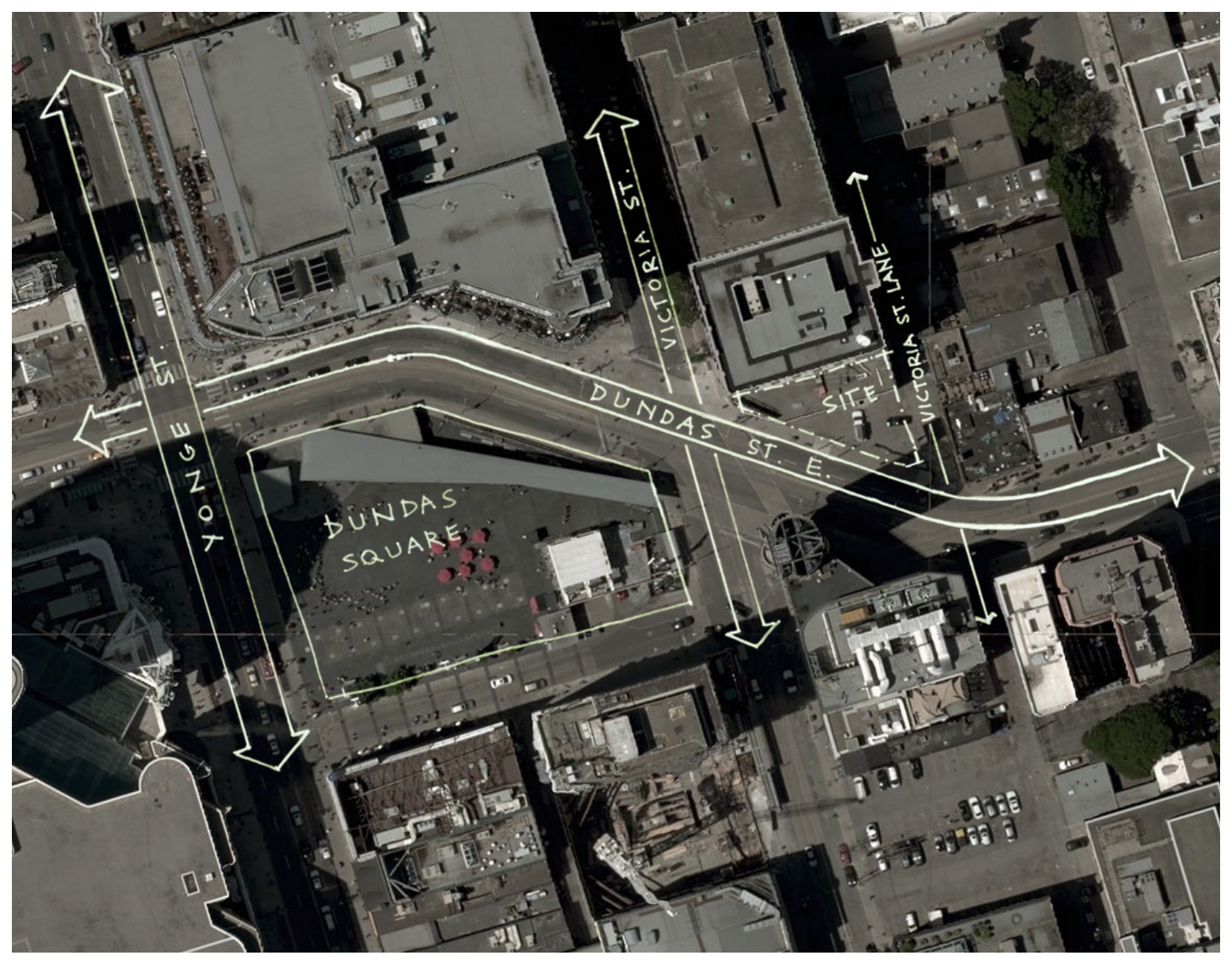

FIGURE 38 MAPPING

Initial Site Analysis 


\section{CHAPTER 10 \\ SITE ANALYSIS}

The site selection emerged from an analysis of Toronto's current communication and transit infrastructure. All major radio broadcasting and film production studios were mapped showing that for the most part they are fairly dispersed across the city. Overlaid are the numerous cell phone towers which connect Torontonians in a very personal level to the media we consume. The cell phone towers seem to follow major arteries and are numerous in other densely populated areas.

After overlaying the information lines of best fit were introduced to locate a central new media communications hub. The point of intersection is approximately at Yonge St. and Dundas St. at Dundas Square. The site is a commercial hub of activity with the northern entrance to the Eaton Centre and a variety of other street level shops and restaurants. The square itself is a vibrant meeting point which constantly hosts a variety of events and is fitted with a stage. The square itself also experiences a perimeter of advertising as most available façade space is occupied with oversized advertisements that are regularly changed. Located on the western edge of Dundas Square at Dundas St. and Victoria St. is an underutilized lot which until recently housed a Hakim optical (currently vacant) and several parking spaces. The site can be understood as the south eastern gateway into the Ryerson campus. 


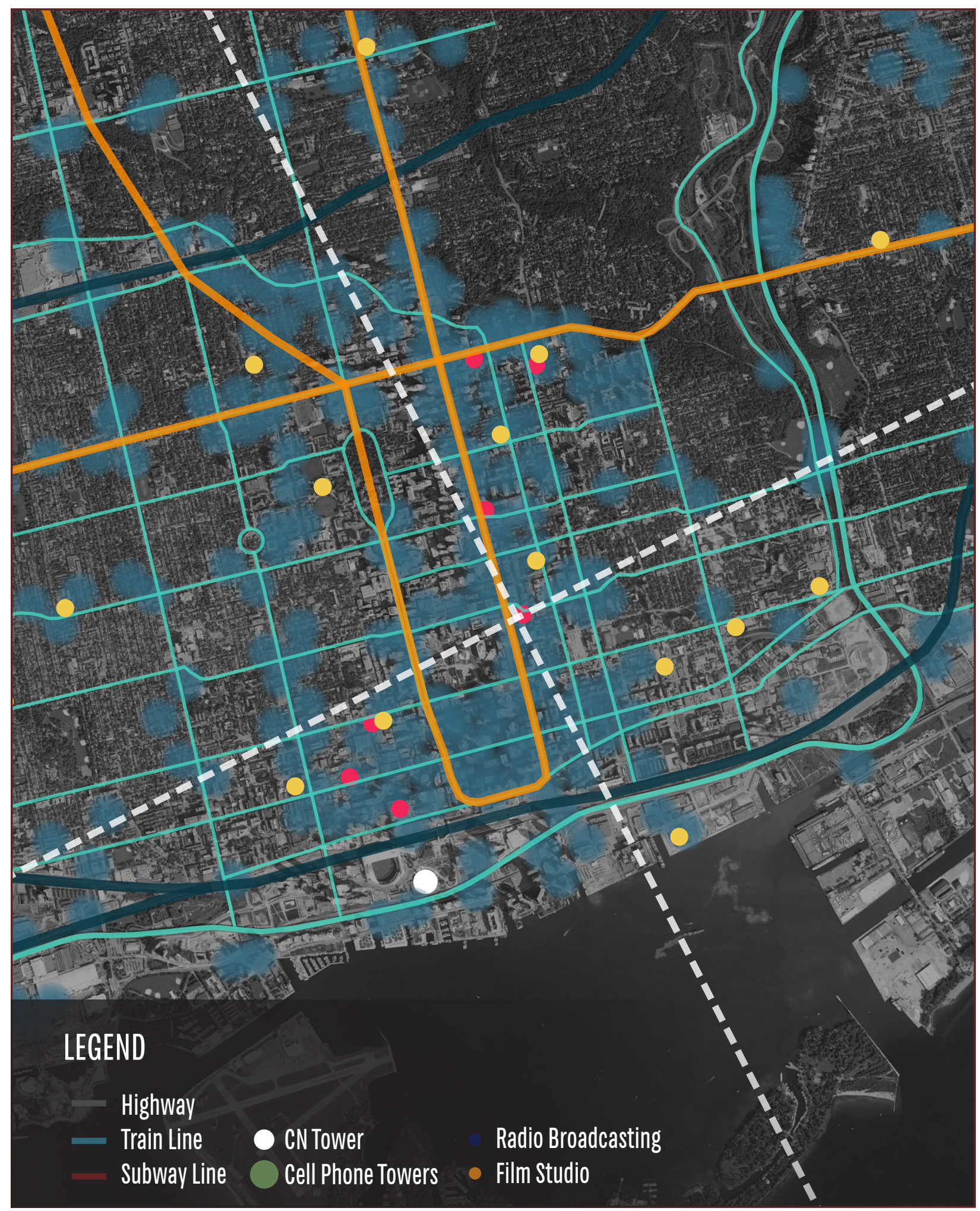

FIGURE 39 MAPPING

Toronto Site Analysis 
CHAPTER 11

\section{SHADOW BOX}

\subsection{SHADOW BOX}

The most definitive aspect of Dundas Square is the wall of advertisements that enclose the square. It is difficult to capture an image of the square void of the flashing lights and competing imagery. Any intervention to the periphery of the square must take into account the impact of the advertising and the architectural implications.

Building off the previous investigation into the layering and augmentation of an image, the notion of a shadow box emerges. The image or artifact of focus is placed into a frame of depth, drawing attention to the image itself. The shadow box provides space for additional filters which augment the base understanding of the image. By bringing the advertisement into the building itself, a space is created that people can occupy. For a school of new media production it brings to the forefront the individuals who are creating the image.

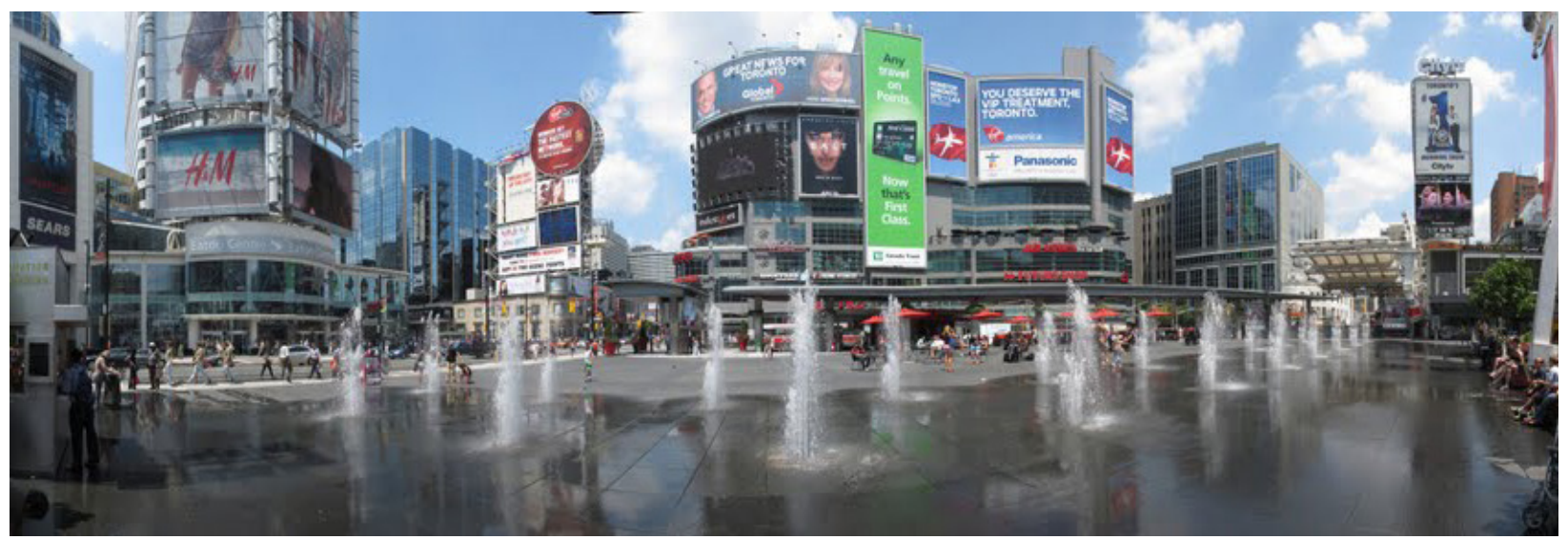

FIGURE 40 PHOTOGRAPH

Advertising at Dundas Square 


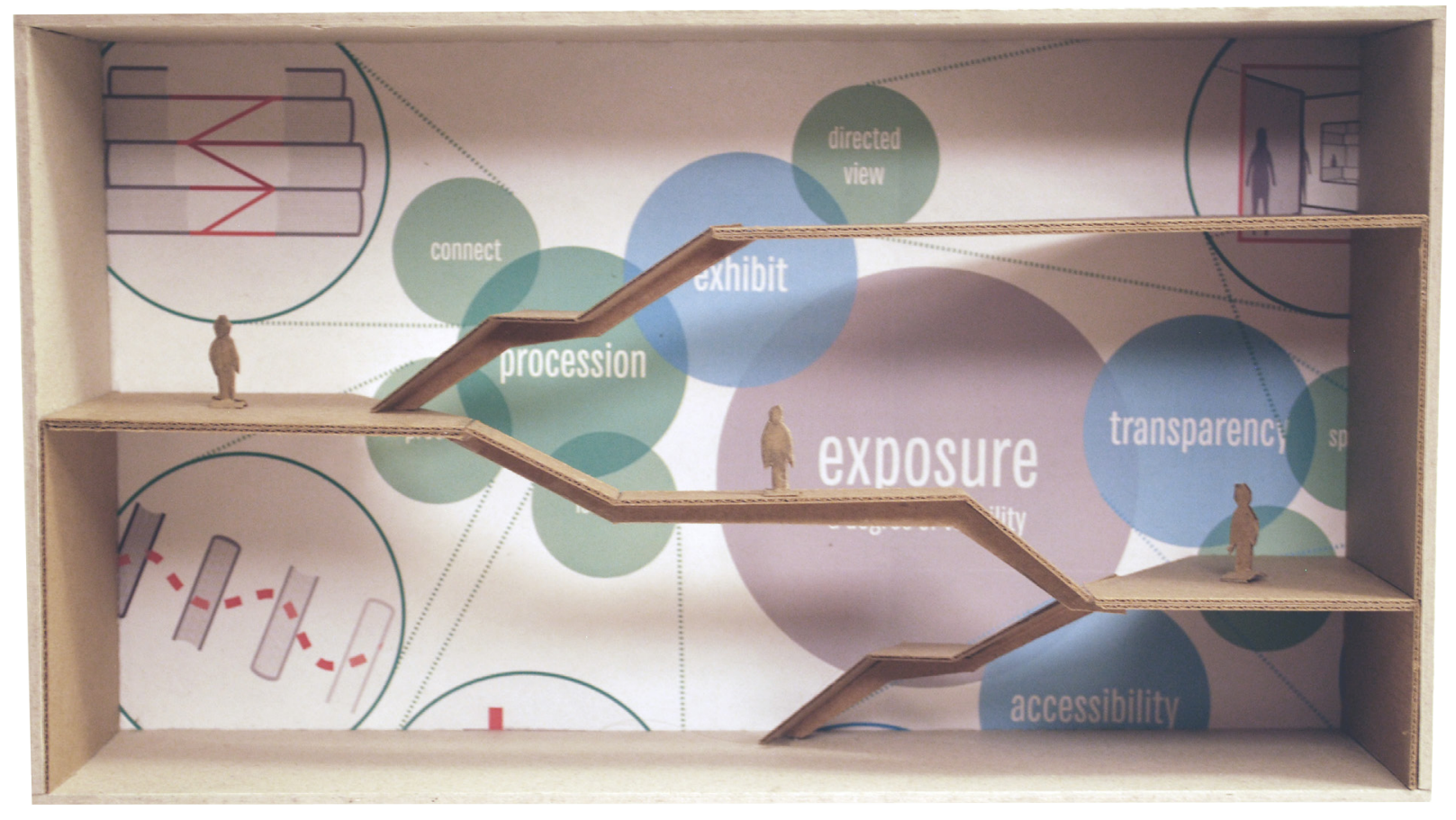

\section{FIGURE 41 EXPERIMENT}

Atrium Scheme Shadow Box

By creating occupiable space it is important to assess the relationship between viewers, the image and the space augmenting the image. Two primary strategies emerged that sees the shadow box as either an 'auditorium' or 'atrium'. In the auditorium focus is placed on viewing. Seating is raked to minimize the obstruction for an individual standing in the square. People inside of the space are superimposed onto the image behind them. The scheme also pays attention to reduce some of the impact internally, drawing most of the views out to the square.

In the atrium scheme focus is on opening up the image to the occupants. This is achieved through the creation of a series of interconnected spaces giving privileged views to each zone, while maintaining a major part of the image unobstructed. As with the previous scheme the users become a part of the image, establishing a level exposure to the process which creates media. 


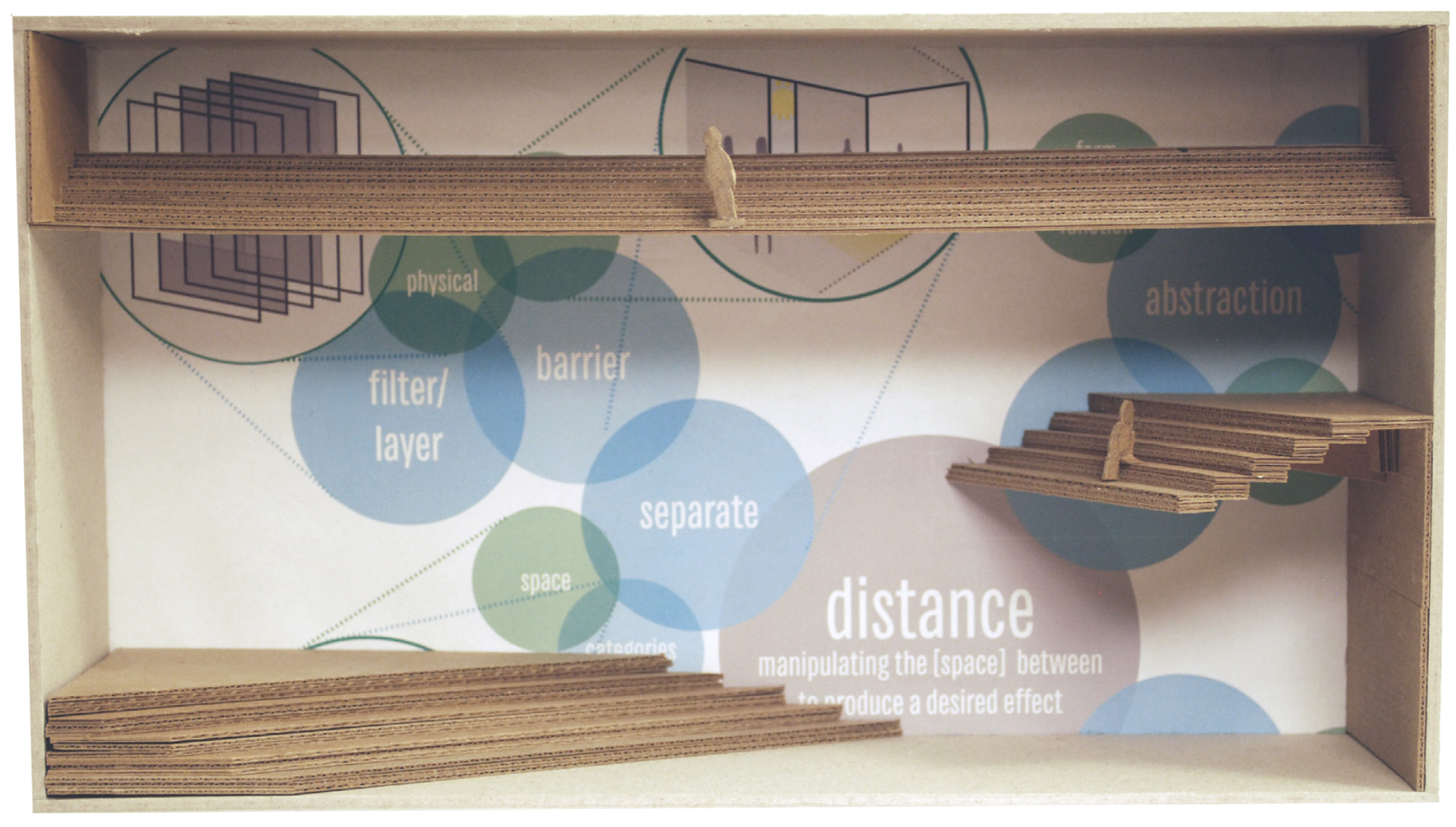

\section{FIGURE 42 EXPERIMENT}

Auditorium Scheme Shadow Box

The models behave more akin to a diagram than a true representation of the space itself. The shadowbox approach successfully creates a sense of isolation. Each image becomes encapsulated in a space with no real connection to adjacent spaces. The image at this point is placed at the highest priority, and because of the isolation creates a series of unique and independent spaces. To a certain degree the connection between viewer and artifact is established but does not read as effectively as desired. The notion of looking is divorced from the models as occupants primarily occupy an ambiguously defined space. If the spaces were able to employ a unique identity that embodied a sense of process, the diagrammatic model might speak more to the act of looking. To emphasize the diagrammatic qualities of the model the image is adequate however an image could be chosen to further reinforce that point such as a still from a commercial or large brand logo. 


\subsection{REALIZING THE SHADOW BOX}

To continue the conversation of the shadow box in a more concrete setting, the idea is translated to proposed spaces within the design. The shadow box at this point evolves into the use of a buffer to obstruct a space, in particular the connection between a mixed media studio and two types of circulation (public and service).

The first iteration, where the length runs parallel to the main circulation, uses large sliding doors to buffer the spaces. The sliding doors create a level of permeability between the two spaces. This opens up studio to the circulation when media is being exhibited and, when possible, showcasing work in progress. With the length perpendicular a vestibule was introduced. The buffer receives users as they maneuver through the primary circulation, drawing them into a space for exhibition. The vestibule acts as the buffer dividing the production and exhibition. Unlike the previous scheme which attempted to subtly expose process, this scheme actively pursues a zone of creation and a zone of exhibition.

Both of these spatial models successfully emphasize a sense of continuity and connection. The enclosed spaces open up to cropped circulation baths. Also the depth of the spaces has become layered. The front elevation shows communal work spaces superimposed onto the entrances of the new media lab. From a plan view the open studio space and its connection to both public and service entrances becomes evident. From a side elevation, the new media lab is seen sandwiched between an open circulation path and a sterile enclosed service path. The service path is disorientating because of the general placelessness, as the user has no visual connection into the studios.

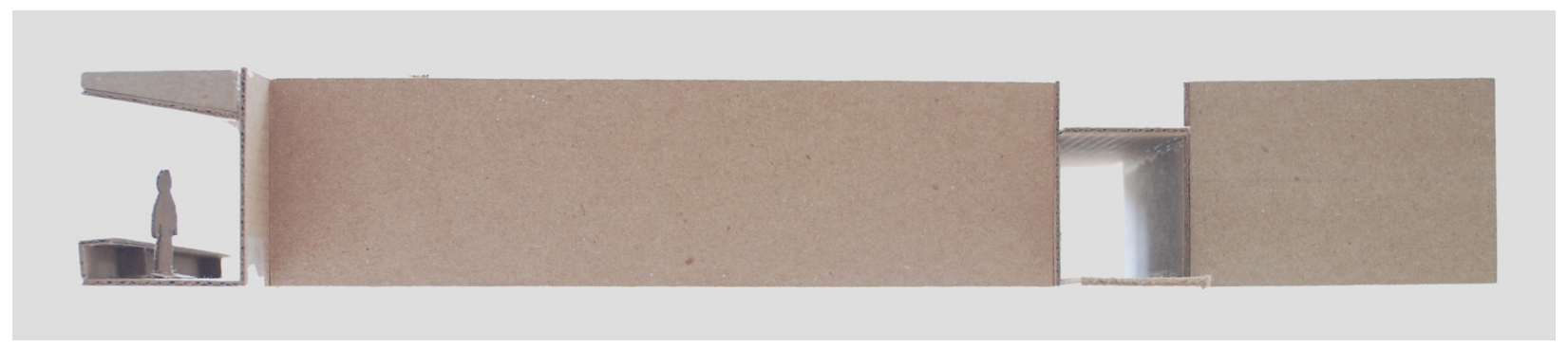

The spatial relationship models showcase a sense of fragmentation through the representation itself. Each model is constructed in a way that emphasizes a connection to a larger building with making no actual reference to said building. This is a complete reversal from the previous shadowbox models which saw very isolated schemes. Although the models are intended to additionally show a layering of spaces, process and exhibition, additional representations may capture that essence more. In particular the ability to explode each component may assist in drawing attention to the buffers that separate them. 


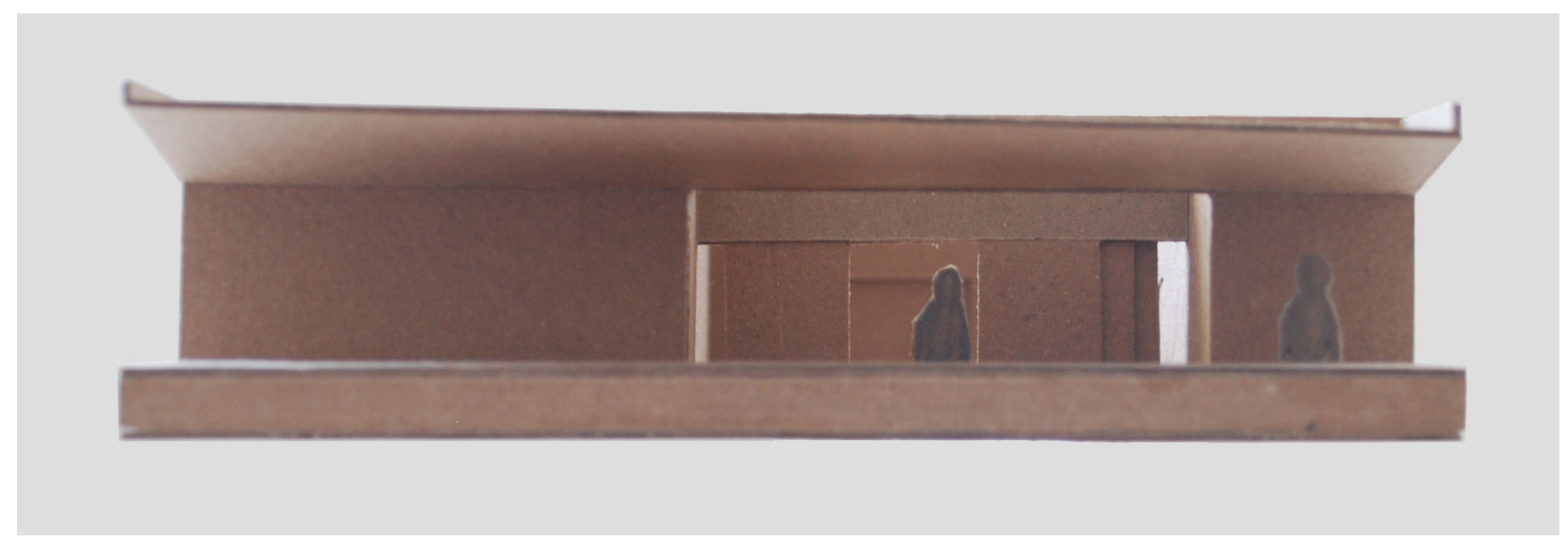

FIGURE 43 MODEL Partial Sectional Shadowbox Model

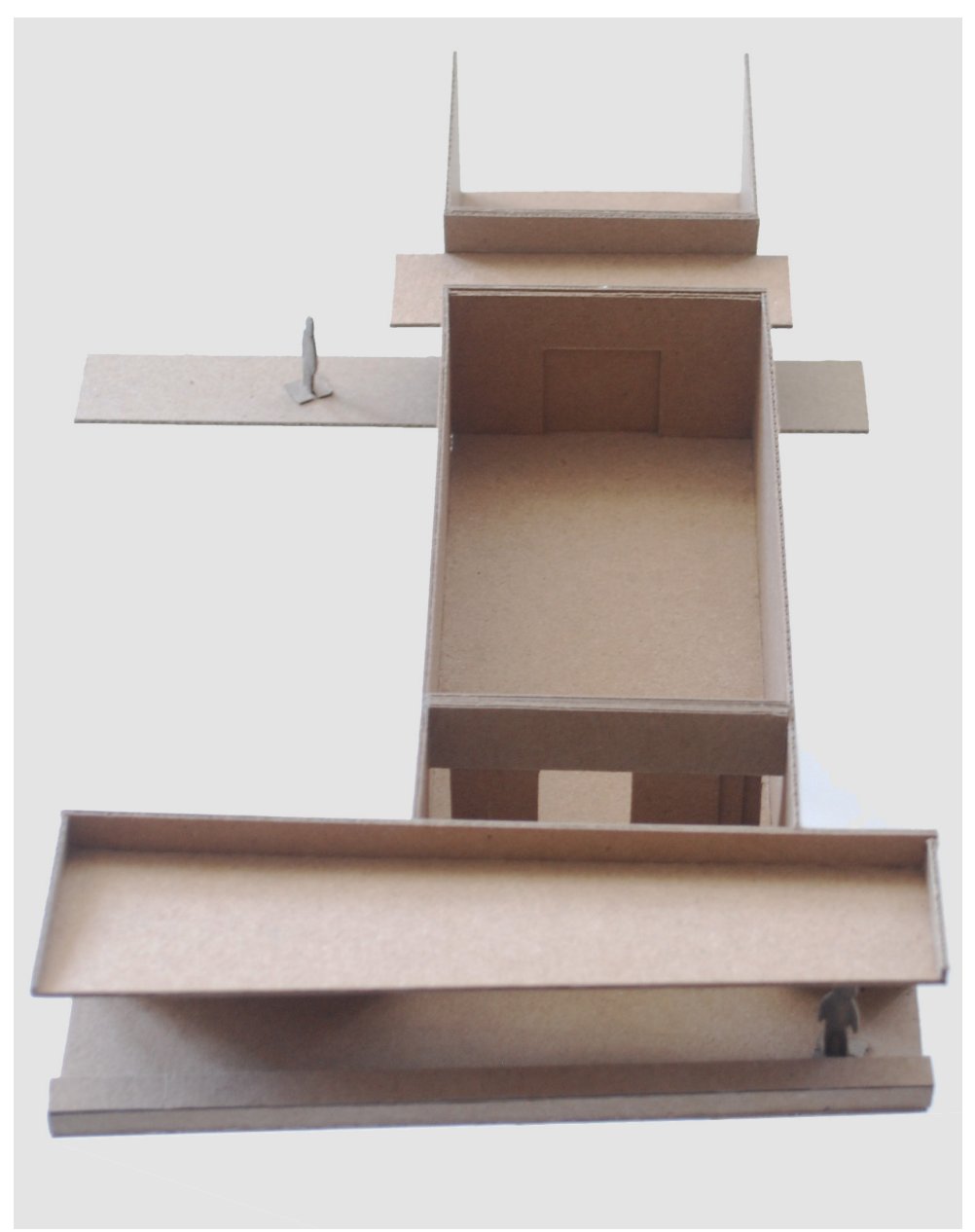



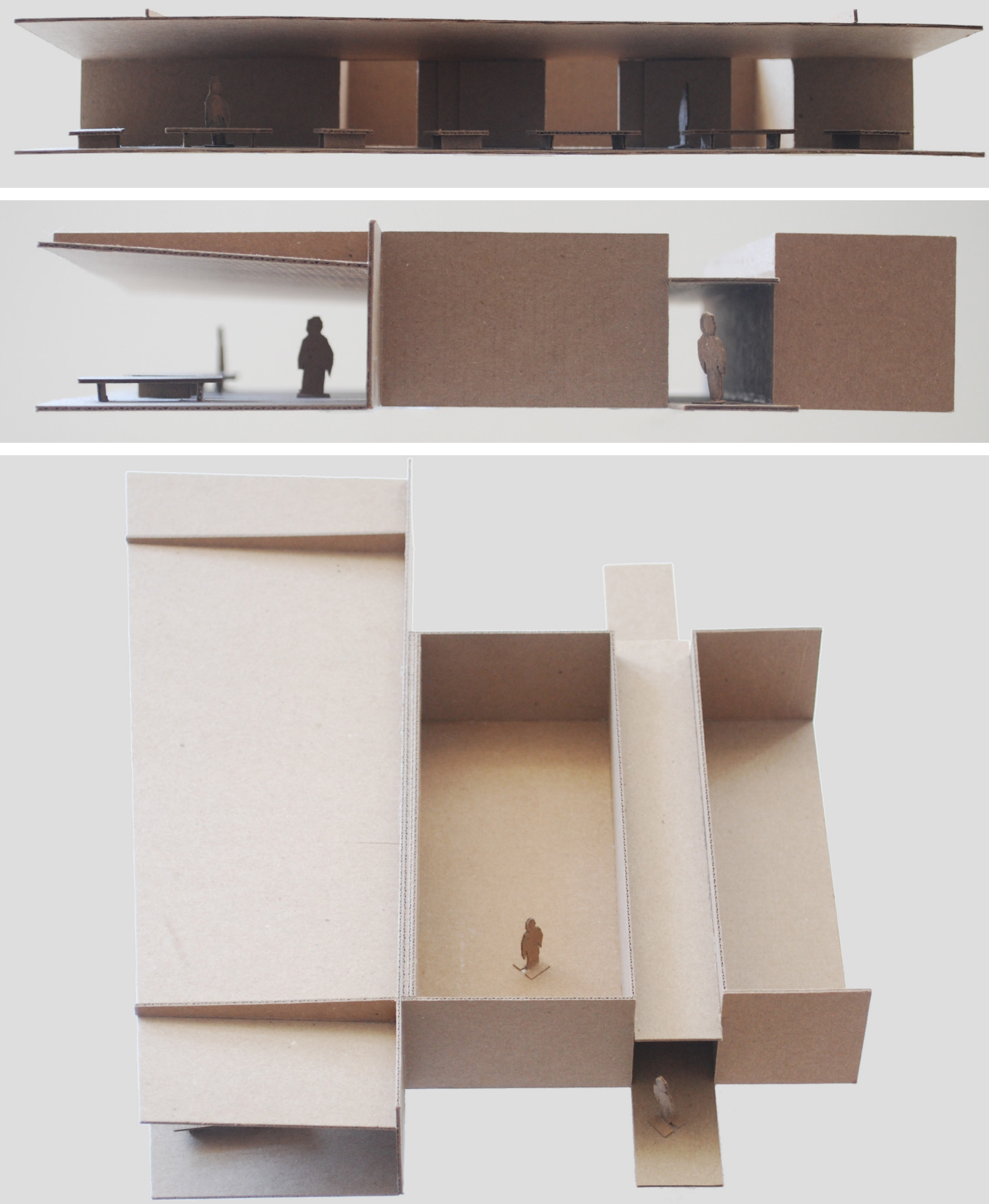

FIGURE 44 MODEL

Partial Sectional Shadowbox Model 


\subsection{CHANGING THE LOCATION}

The following models revisit the theme of looking and the ramification of the aperture. The investigations are located between a breakout space and a sound stage. The raked seating is focused along an aperture looking into the sound stage itself. This creates a space with strong connectivity to the sound stage, as users are able to engage with work under development. Additionally the aperture bridges the two spaces, the stage which is highly enclosed and controlled, with the second periphery space which is very open. In the second model the inclusion of seating raked in the other direction focuses a view away from the stage. This maneuver frames a part of the sound stage wall, drawing the eye to the middle of the space when looking from the outside.

Although the model attempts to mimic the conceptual language of connectivity achieved in the previous investigation, the overall clarity is lacking. The models to a certain degree of efficiency are capable in developing a sense of continuity. The correlation between the models and looking is unclear because of the overt ambiguity of place.
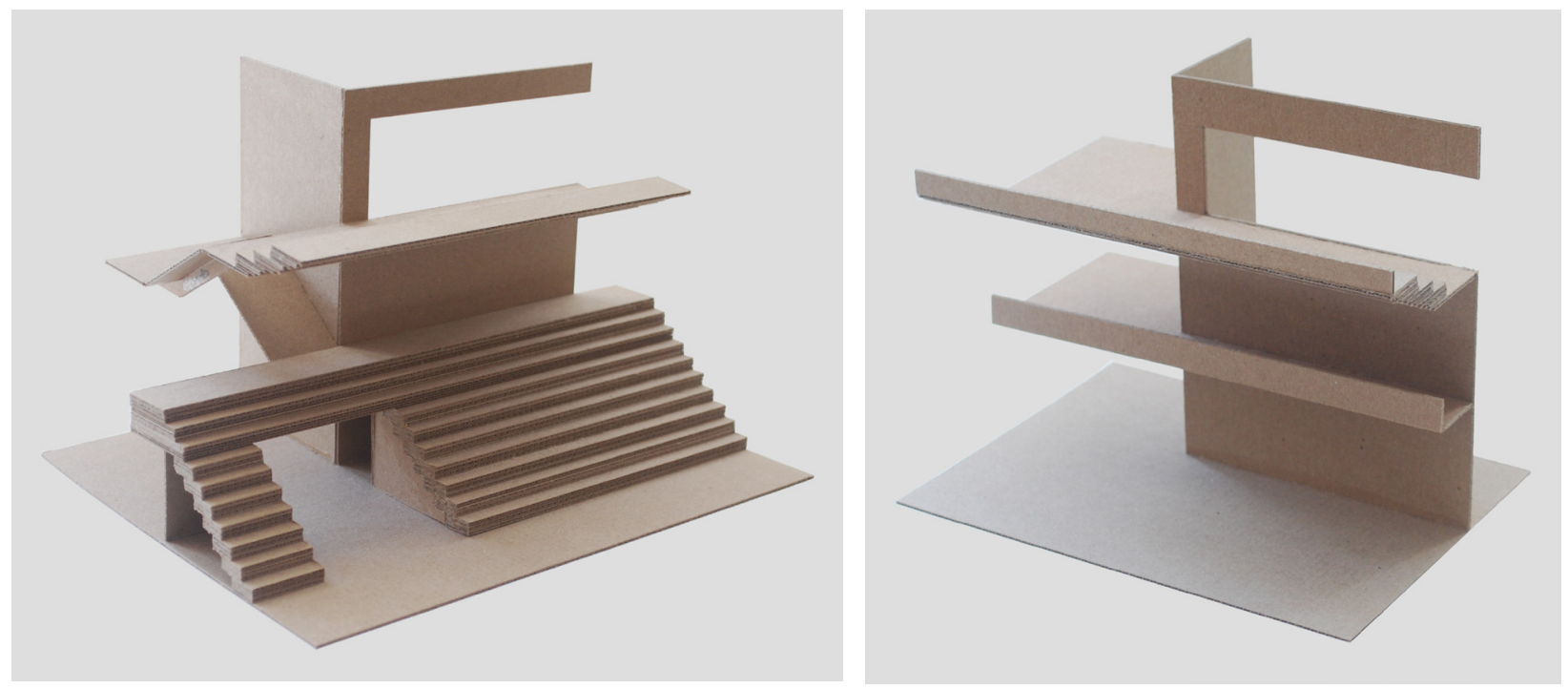

FIGURE 45 MODEL

Partial Sectional Shadowbox Models 

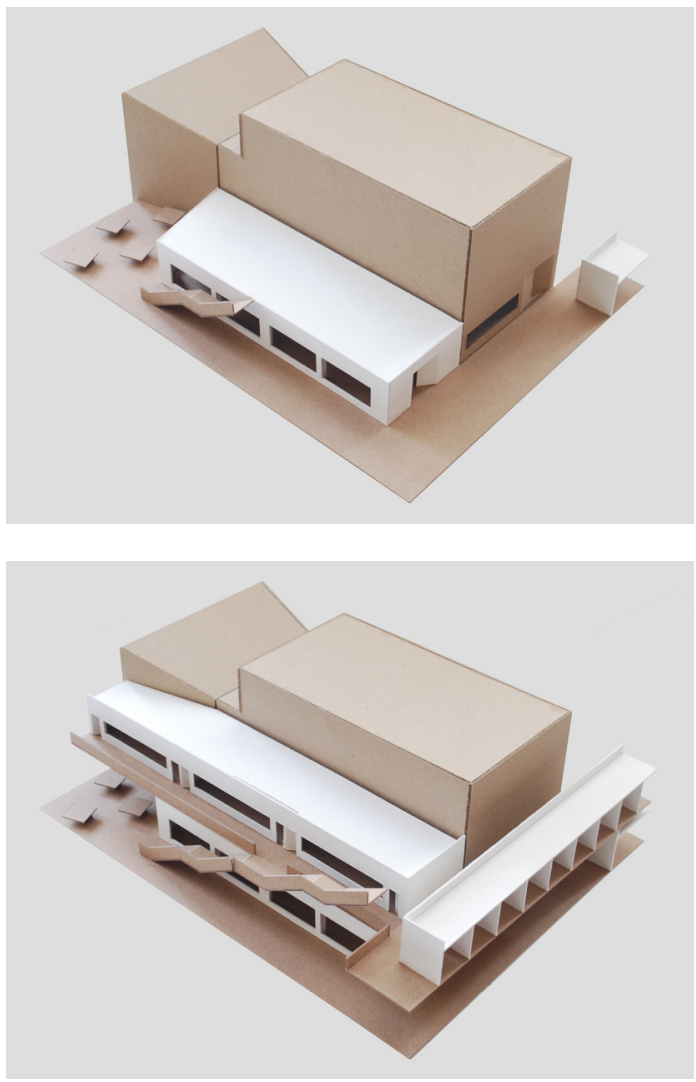

FIGURE 46 MODEL

Experimental Sectional Model

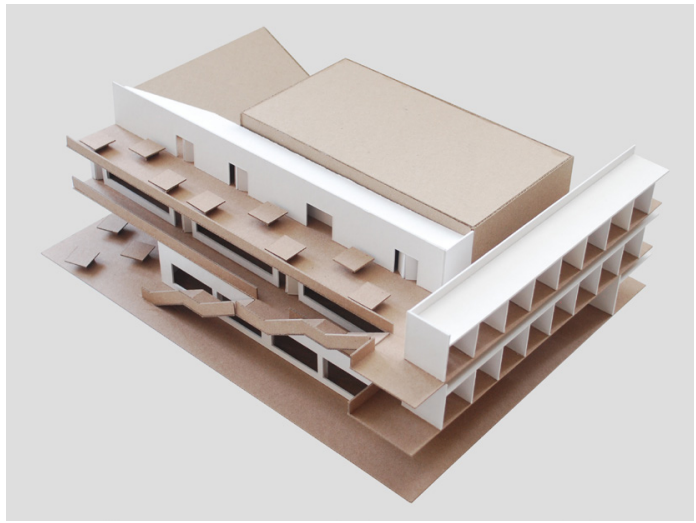


CHAPTER 12

\section{SECTIONAL PARTI MODEL}

Following the failure of the previous models overall spatial ambiguity this model aims to redefine the relationship between theatre and surrounding spaces. Two primary concepts are exemplified. The theatre with the shadowbox examine the relationship between viewing and being viewed, while the circulation and axillary spaces speak to exposure through process.

The sound stage and shadowbox question viewing and being viewed. The raked seating focuses the attention onto the screen, leaving it largely unobstructed. The bottom raked seating looks out onto the square, leaving users to subtly occupy the image and provides an entrance into the sound stage through the screen. Also the tiered seating at the top focuses a view into an aperture looking into the sound stage. Similar to the previous model this space and aperture draws a strong visual reference into the process of production.

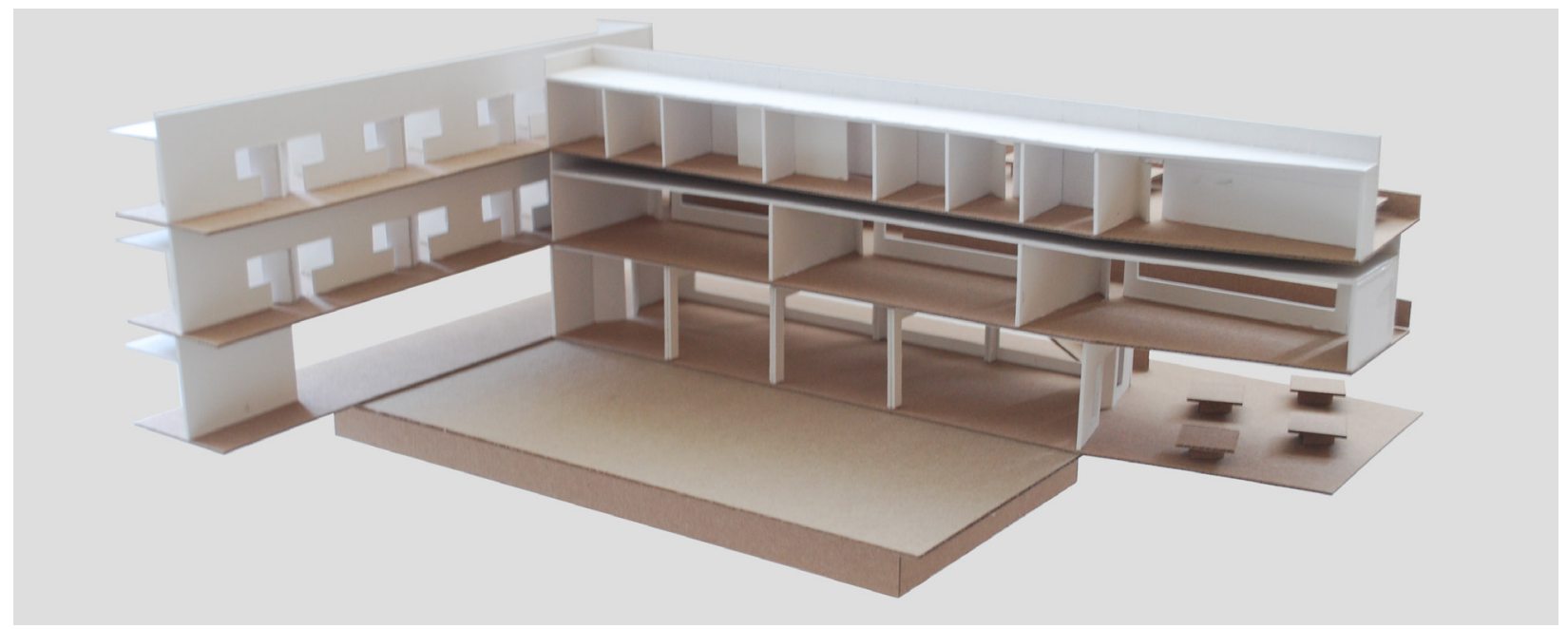

FIGURE 47 MODEL

Experimental Sectional Model, Soundstage Removed 


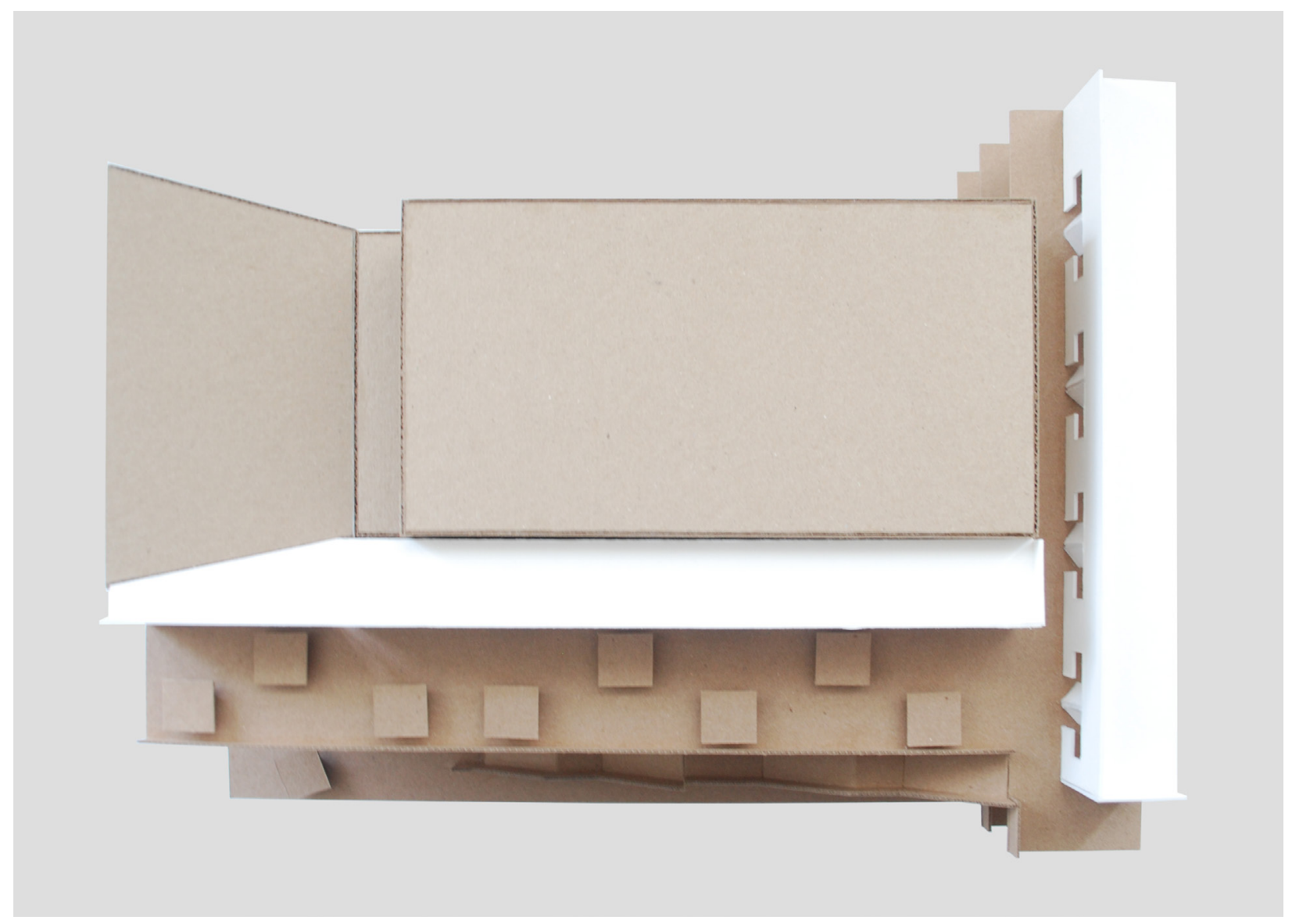

FIGURE 48 MODEL

Partial Sectional Model

Exposure through the process of production becomes evident as the viewer makes their way through the primary circulation. At the bottom level is a workshop with large apertures. As the workshop does not require a visual privacy it allows a passerby to readily view stages and props under construction. Moving up a floor are 3 multipurpose lecture rooms. The spaces require some visual seclusion however remain relatively open to the outside. On the top level are several post production suites that are very inclusive. These spaces require a high degree of separation from one another and as such are located adjacent a communal work/circulation space. This is done to increase the amount of interactions while giving the individual suites privacy. 


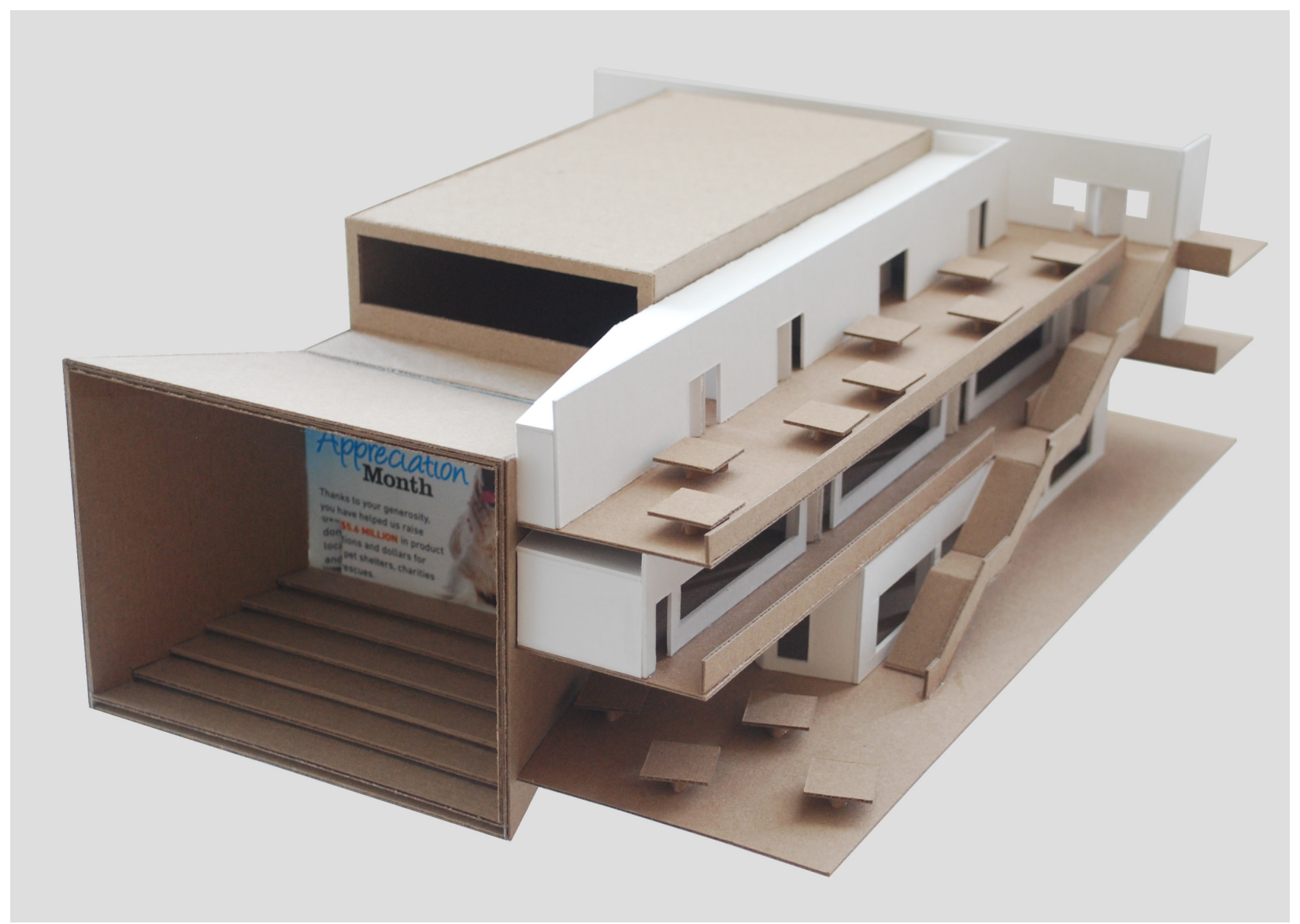

FIGURE 49 MODEL

Partial Sectional Model

In learning from the previous models the model was constructed in different materials to differentiate the sound stage from supporting spaces. The model is also a section in every direction, making reference to the connectivity and fragmentation from the second study models. The tight bounding box of the model is capable of visually implying connections to other spaces, allowing the viewer to complete the image. In creating a tangible spatial composition some downfalls of previous investigations are quenched, allowing to model to more accurately represent some of the larger ideas. Finally the ability for the model to open up into 4 distinct pieces (each floor and the sound stage) allows a sequence to emerge, showcasing how a user would begin to operate through the individual layers. 
FIGURE 50 DIAGRAM Massing 1.1
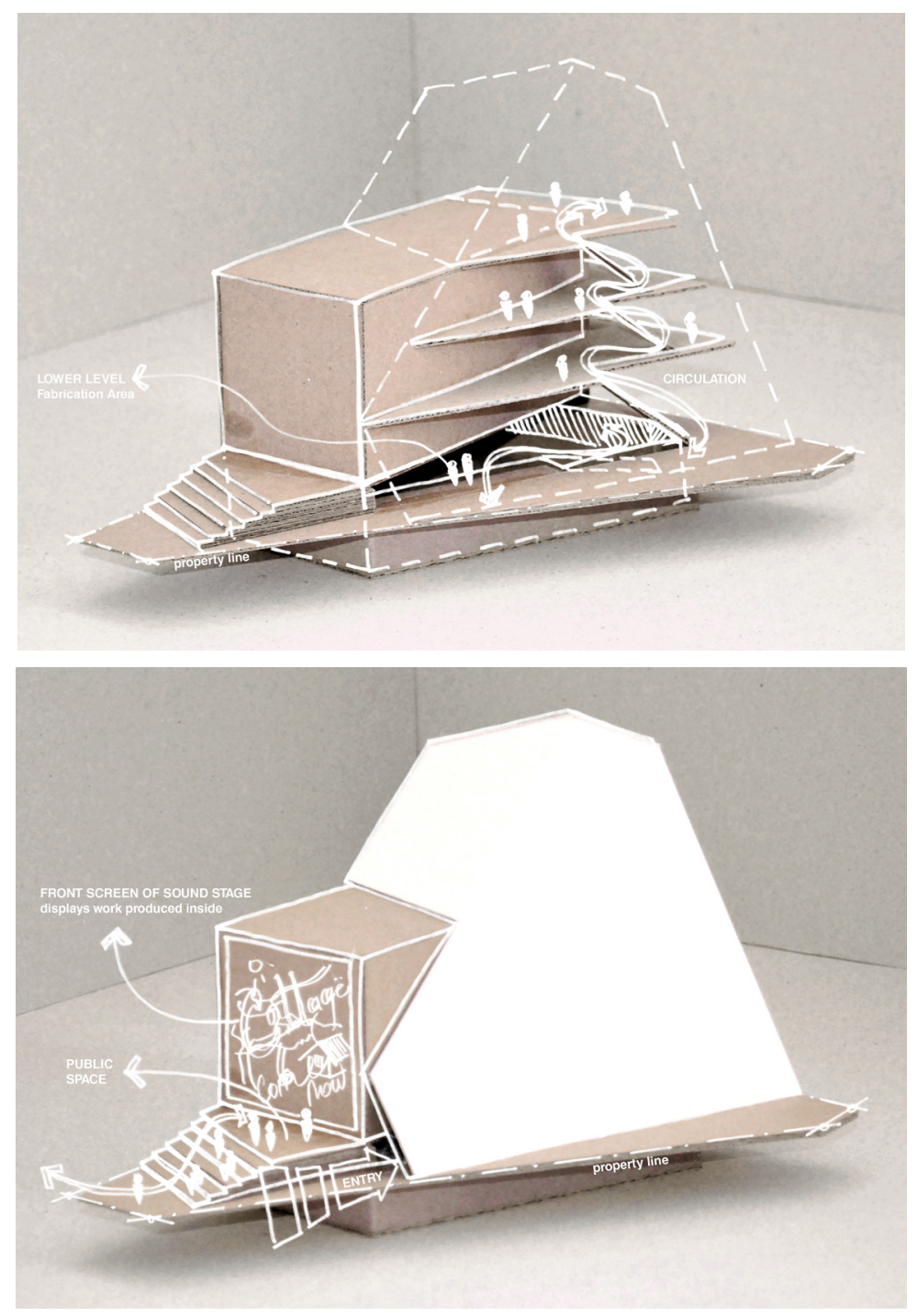
CHAPTER 13

\section{DESIGN 1: CIRCULATORY NARRATIVE}

\subsection{MASSING}

From the previous conceptual iterations it became evident that the initial scope was too generous and that the ideas can be further explored on a smaller scale. The following building design iterations aim to grasp two of the primary drivers in the previous conceptual iterations; the exposure of process through circulation and the threshold explored in the shadow box.

The wedge shaped plot on the southern corner of Mutual and Dundas streets requires any structure on the site to address views to and from Dundas Square. Additionally the visual dominance of advertising in the square itself must lend itself to the Ryerson University New Media Production Centre, allowing the school to participate in this dialog and connect the city to the work of the students.

1.1 In the first iteration I was successfully able to create a strong juxtaposition between the artifact and process. The sound stage emerges from the multifaceted process spaces, with ramped seating and a large projection surface. Once inside the patron moves through a series of floors where the various programmatic elements are located; starting with an exhibition space adjacent a workshop below grade and moving through discrete spaces such as post production rooms until they are at the open communal workspace at the top floor.

In this iteration presentation is symbolically separated and emerging from production, developing a focus on the sound stage as a presentation space. This however is awkwardly handled on the ground floor as people aim to occupy the space directly below while looking out onto Dundas Square. Additionally the circulatory narrative is underdeveloped, in turn directing and being directed by the overall form. The production spaces thusly feel arbitrarily defined and the entrance requires a more development. 
1.2 The second iteration begins with a more thoughtful understanding of access into the facility. The sound stage is raised up onto the second floor creating an overhang on the southern entrance. The space beneath the sound stage doubles as a presentation space and auditorium style seating. As the first node of the circulatory narrative it begins by showcasing gallery style space/ screen and the work shop on the same floor. Moving up through the building one again sees the juxtaposition between process and presentation as the sound stage is constantly visible.

This iteration is successfully capable in addressing the entrance and the starting node for the circulatory narrative. Additionally the sound stage is used again to create a formal juxtaposition that reacts to the site boundaries. As with the previous iteration the later aspects of the exposure of process through circulation become lost in a general ambiguity that can be resolved in a more rigorous development of program.

In having the primary formal focus on the juxtaposition between the sound stage and production spaces the initial concept of the shadow box becomes under developed and lost. In the previous iteration it reached a certain degree of success with the raked seating on the western façade, however in internalizing the dynamic in this scheme the shadow box was lost. Future iterations can begin to understand how other programmatic elements can act as the shadow box and strengthen the circulatory narrative. 
FIGURE 51 DIAGRAM Massing 1.2
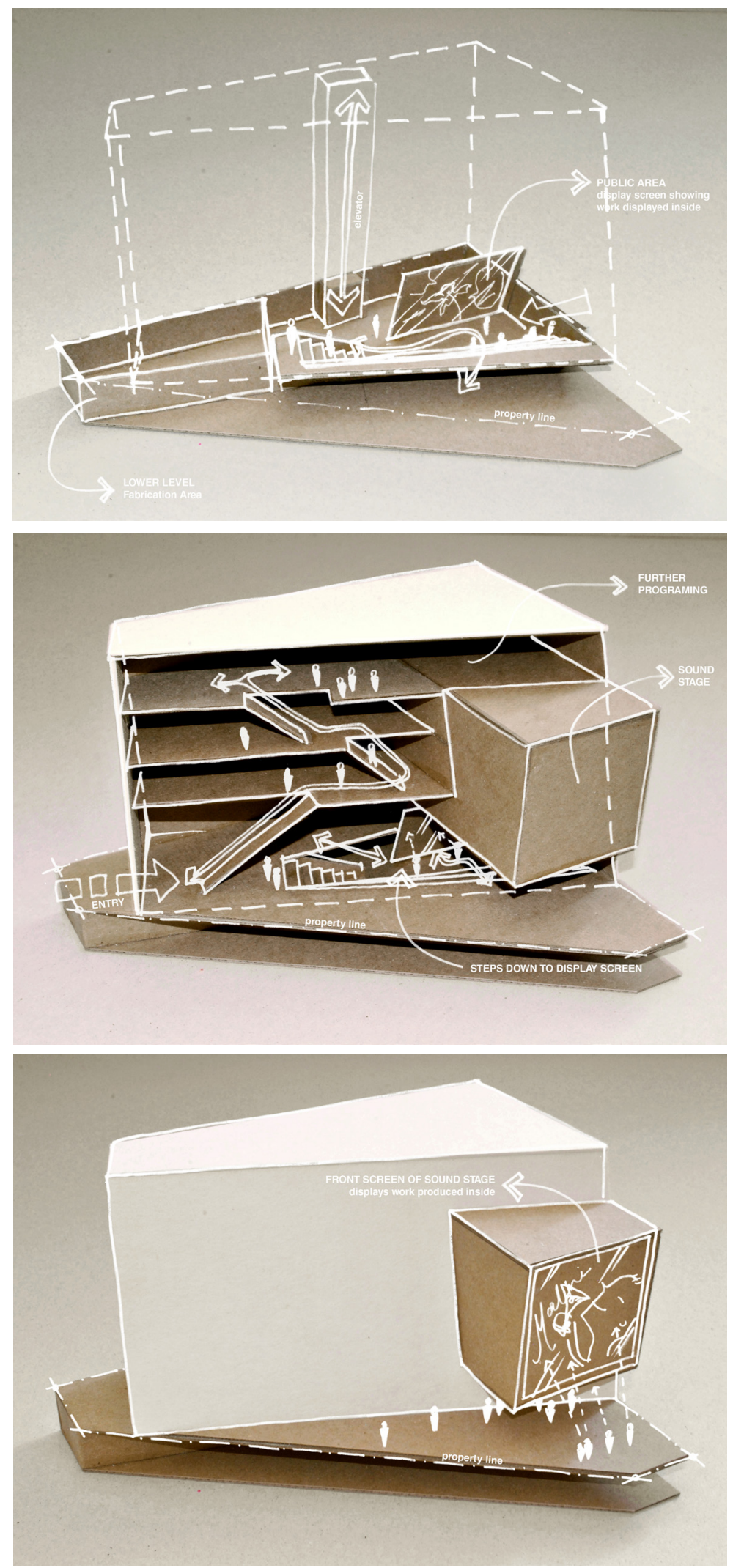
FIGURE 52 DIAGRAM

Massing 1.3
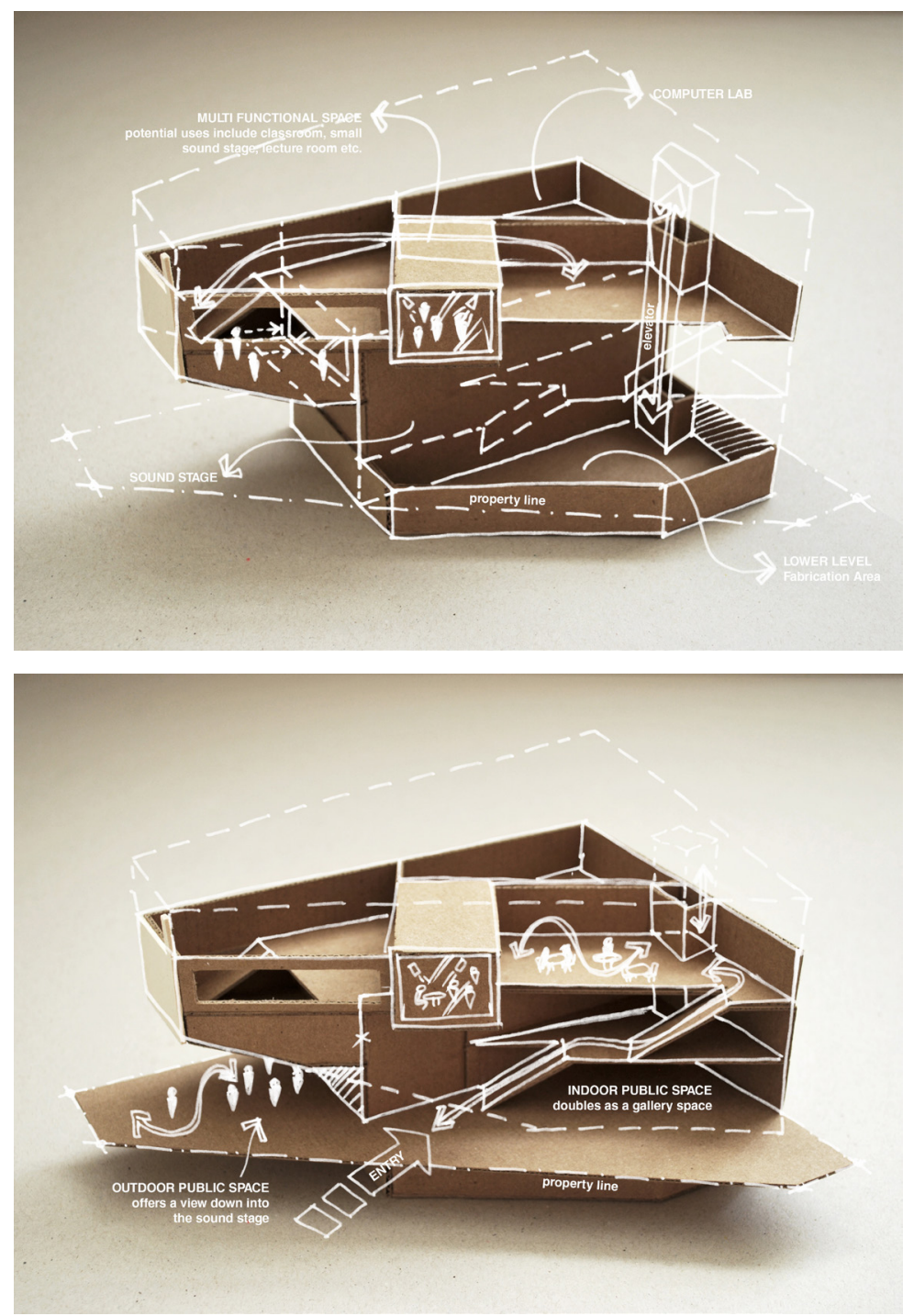

1.3 The third iteration sees a shift in visual dominance from the main sound stage to the smaller new media labs has reinforced the shadow box as it was initially explored. Each new media lab becomes a portal protruding from the façade showcasing individuals at work. Additionally reintroduced to the iteration is the raked seating view into the main sound stage from the previous sectional model. In this iteration the sound stage is centrally located, straddling the interior/exterior threshold at the western façade.

The circulatory narrative of exposure through process is further concretized in this iteration. The user is initially drawn to the below grade workshop space and sound stage (the rawest input) and then moves up through the other moments of post-production. The second major node can be defined as the computer lab and new media lab. Adjacent these spaces is a second atrium at its base has the view into the main sound 

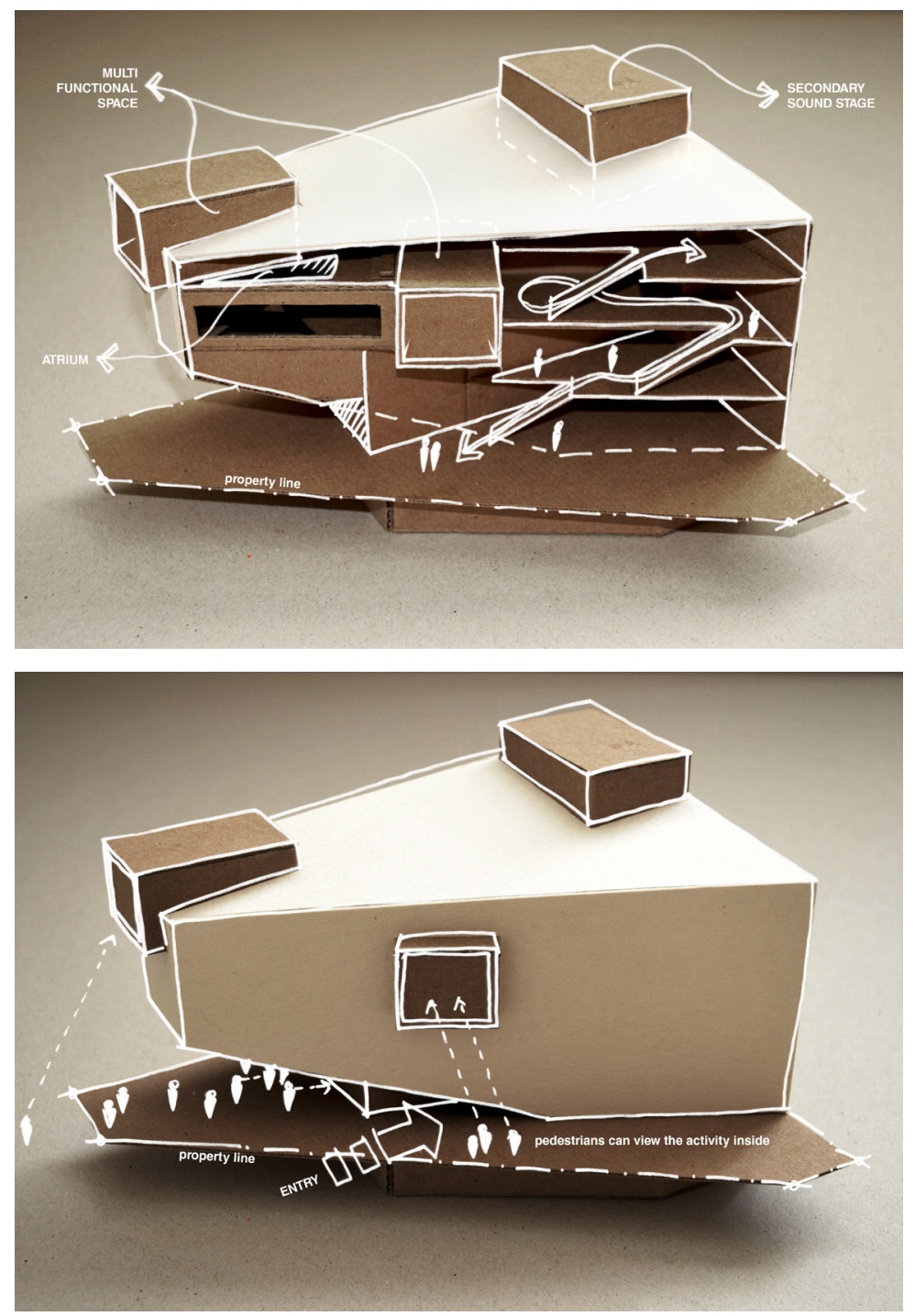

stage. Moving up another floor are additional new media labs, communal work space and room for postproduction suites.

In clarifying the programme and continuing a focus on the individual spaces and nodes, the circulatory narrative becomes more evident. Simultaneously however the present awkwardness of the circulation route is noted (doubling back to enter the space for viewing the sound stage) and future iterations must address the continuity of the horizontal process layering. 
FIGURE 53 DIAGRAM

Massing 1.3
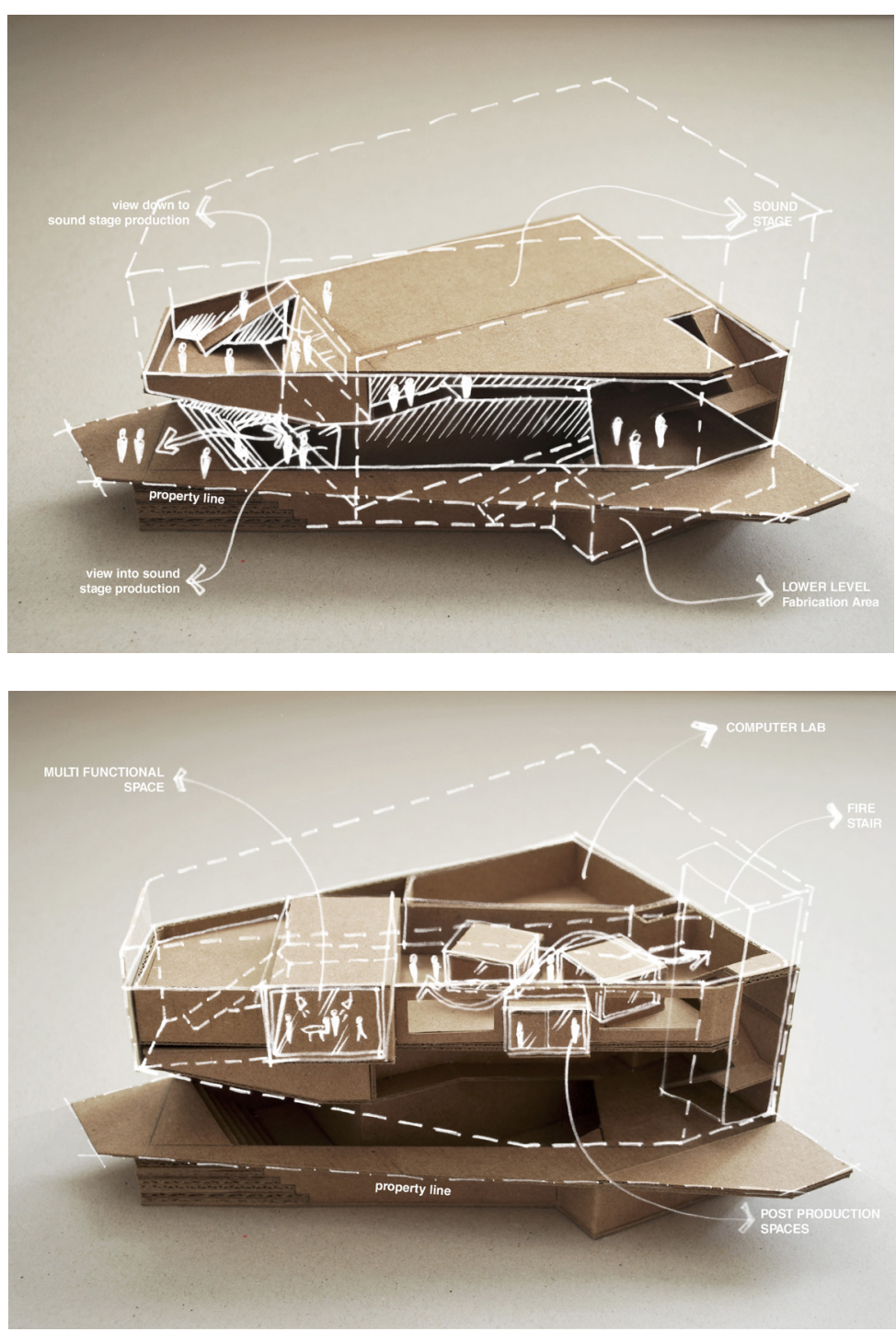

1.4 The primary elements that have been developed in this iteration is the refinement of the post production suites and the introduction of two circulation paths. Drawing from the second iteration and third iterations, the sound stage is sunken below grade and a raked seating is used to place emphasis on the internal west façade of the sound stage. This creates the first major node of exhibition that lures the patron further into the space and to the sound stage itself along with a visually unobstructed workshop space. This point experiences a forking in circulation routes with one stacked stairwell drawing users up through the floors and the other winding users through spaces. The next node is an auditorium style space that overlooks the inside of the sound stage. After this space the user enters an open floor wedged between a multipurpose new media lab and computer lab. This open work area has several post production pods anchored to a vertical threshold. This is done to create a vibrant communal space around the post production pods which 

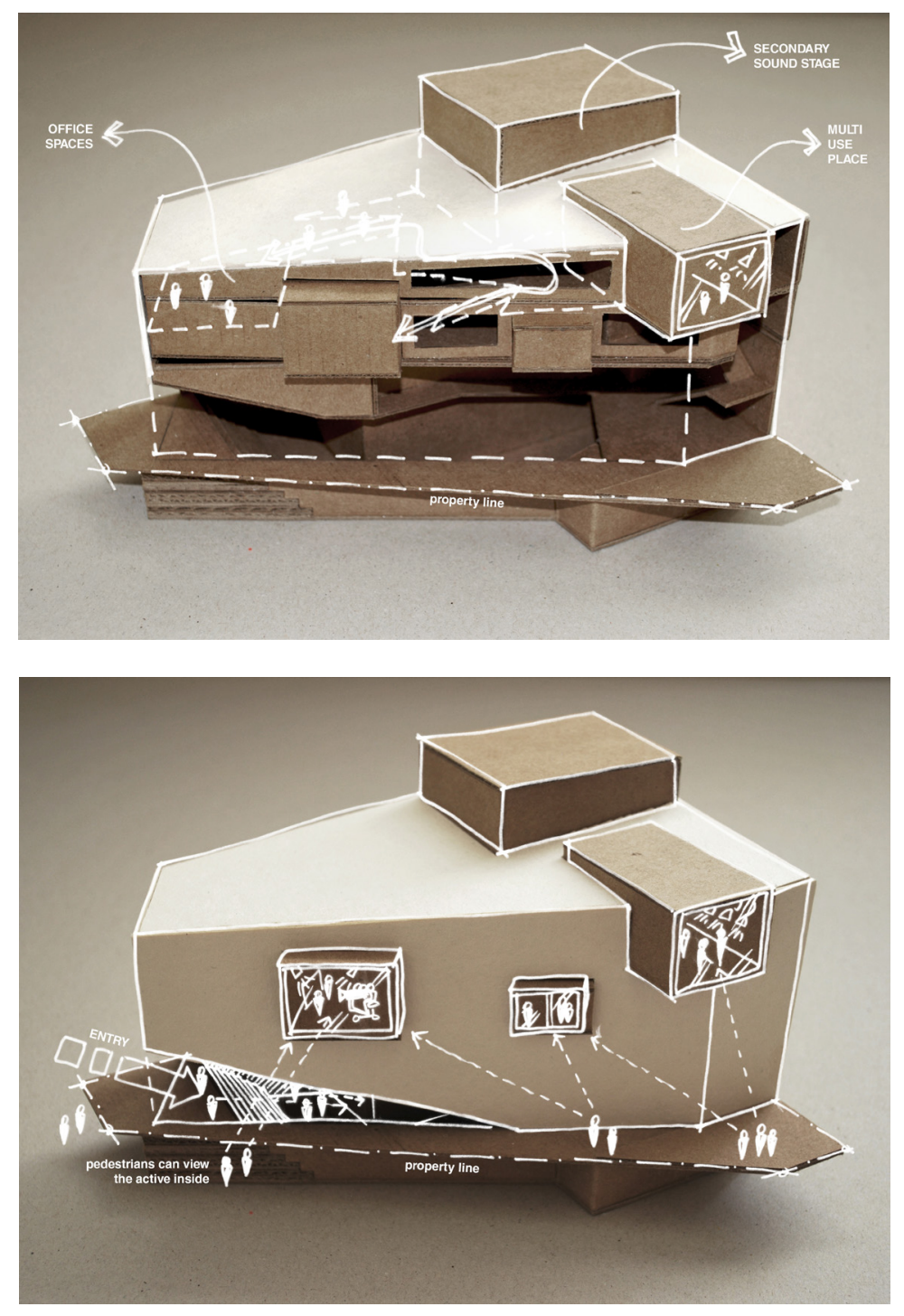

are inherently enclosed. The top floor has an additional new media lab and a second smaller sound stage which can easily double as a gallery space.

In this iteration the circulation narrative is clearly defined and establishes 5 major nodes for exposing process. As with the previous iteration the shadow box can be understood as the protruding new media labs and post production spaces, however the spatial implications afforded by these unique thresholds is underdeveloped at this scale. In changing the scale the relationships between these thresholds can be more thoroughly examined. 
1.5 When looking at each of the design iterations some of the site constraints become evident. Each iteration to a certain extent wrestles with the overall wedge form of the site, and begins to erode and evolve to accommodate programme. Each massing aimed to solve the same initial requirements and through the refinement of the programme was able to evolve with complexity. The narrative of the spaces has been instrumental in sculpting the form and defining the overall character of each massing.

\subsection{PARTIAL MODEL}

By increasing the scale and exploring the upper production spaces what becomes evident are the creation of unique moments and areas for further exploration. From the exterior the multi-purpose new media labs behave as portals piercing the façade and, due to their increased floor to ceiling height, provide interesting view ports into the space from the interior. This becomes particularly evident in multi-purpose new media lab adjacent the viewing auditorium. The ramped access to the office area cuts through the upper portion of the space offering a highly privileged view into the lab.

The model begins to isolate the different zones of production and exhibition, creating a lively public space surrounding the post-production pods computer lab. The zone additionally opens to the upper floor where the second sound stage/ gallery space is located and the other new media lab. Continuing the gradual deconstruction of process from the initial entrance screen through the various post production spaces is the final gallery/sound stage node on the top floor.

It becomes evident in this model that focus must be placed on the individual narrative nodes themselves and not on the overall building. This will clarify the narrative procession and leave room for further analysis on the space between each node before returning to compile the narrative. The initial spatial explorations can be understood at this point as a series of episodes which were compiled into a narrative journey in the massing explorations. The purpose of increasing the scale is to assess and elaborate on each episode, furthering their association with the circulatory narrative. 

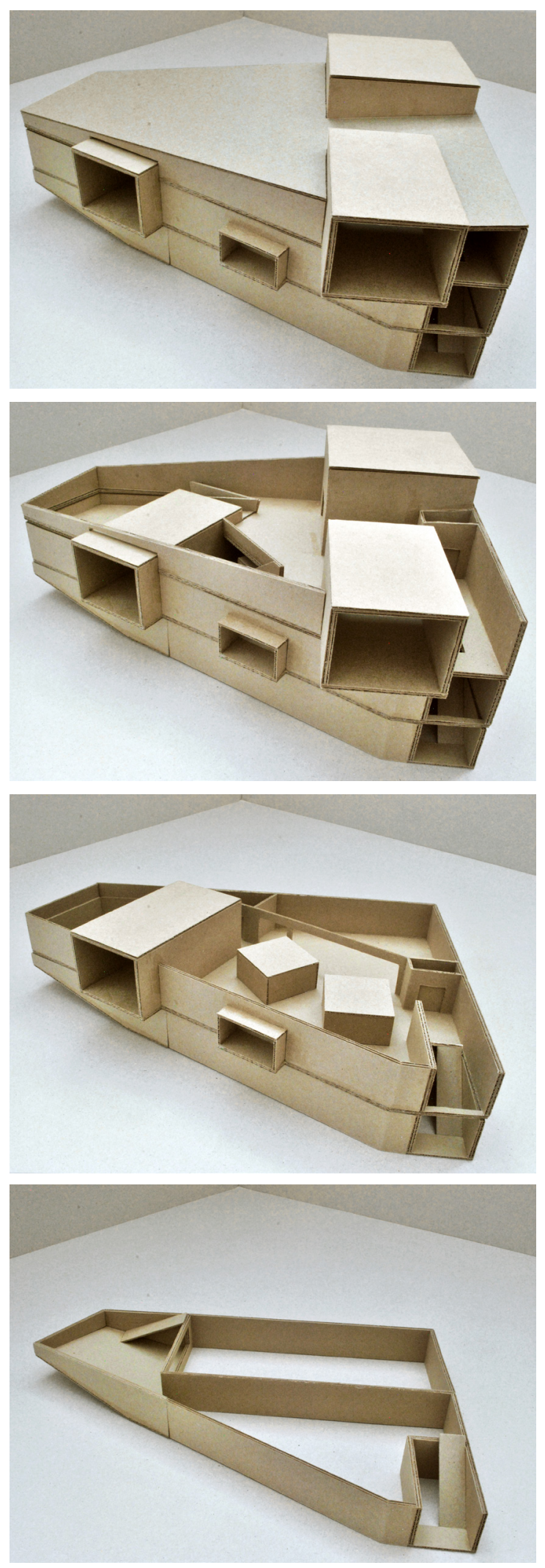

FIGURE 54 MODEL

Partial Model, Opened Up 


\subsection{ENTRANCE EXHIBITION VIGNETTE}

The western entrance focuses on drawing users into the building, creating auditorium style seating overlooking the westernmost wall of the sound stage. This allows for a vibrant public space with the potential to exhibit live streams and clips of current work. The sloping soffit limits the evening light and glare that would penetrate the space. Additionally under the soffit are 3 large rotating doors creating a permeable threshold

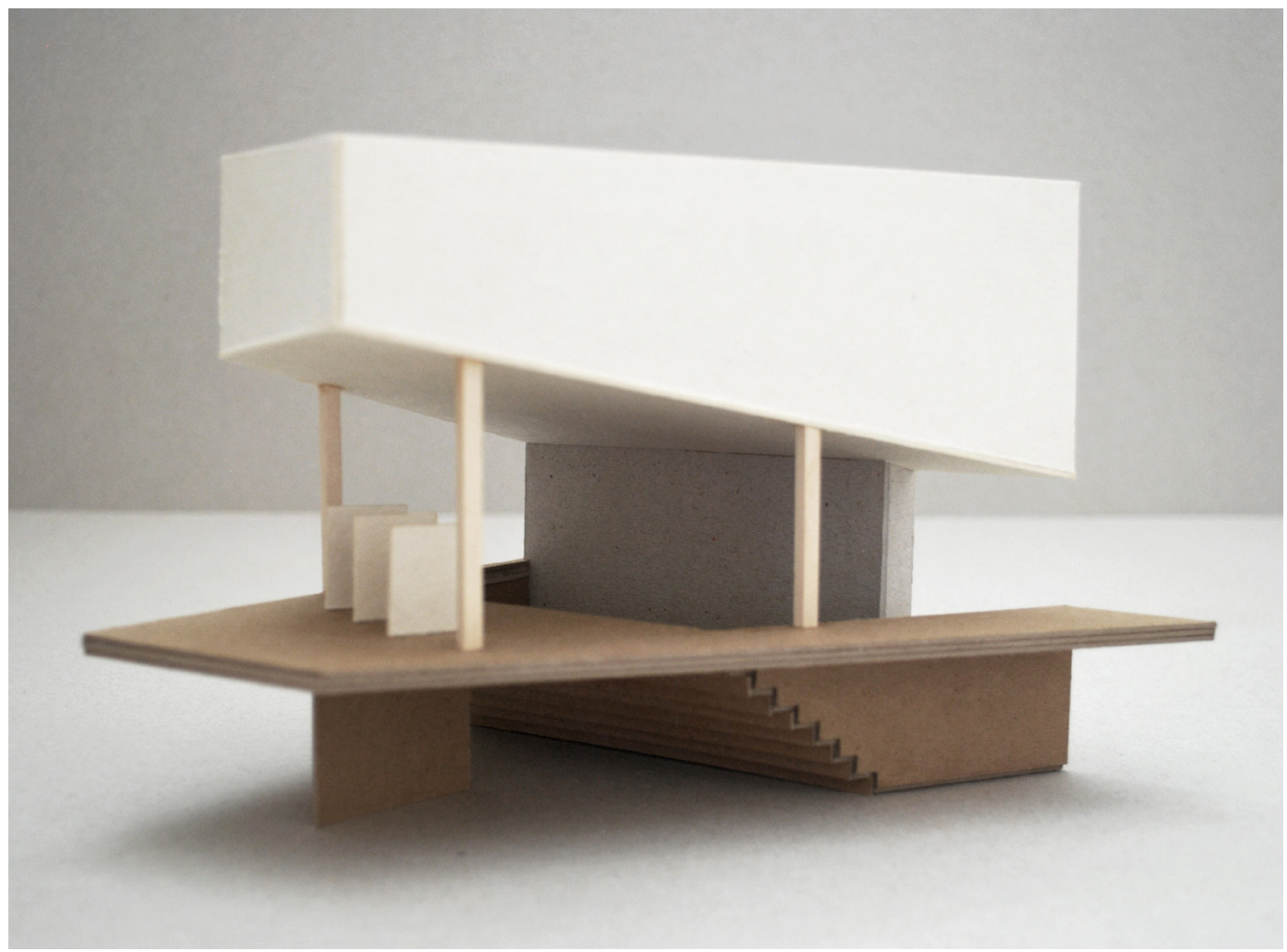




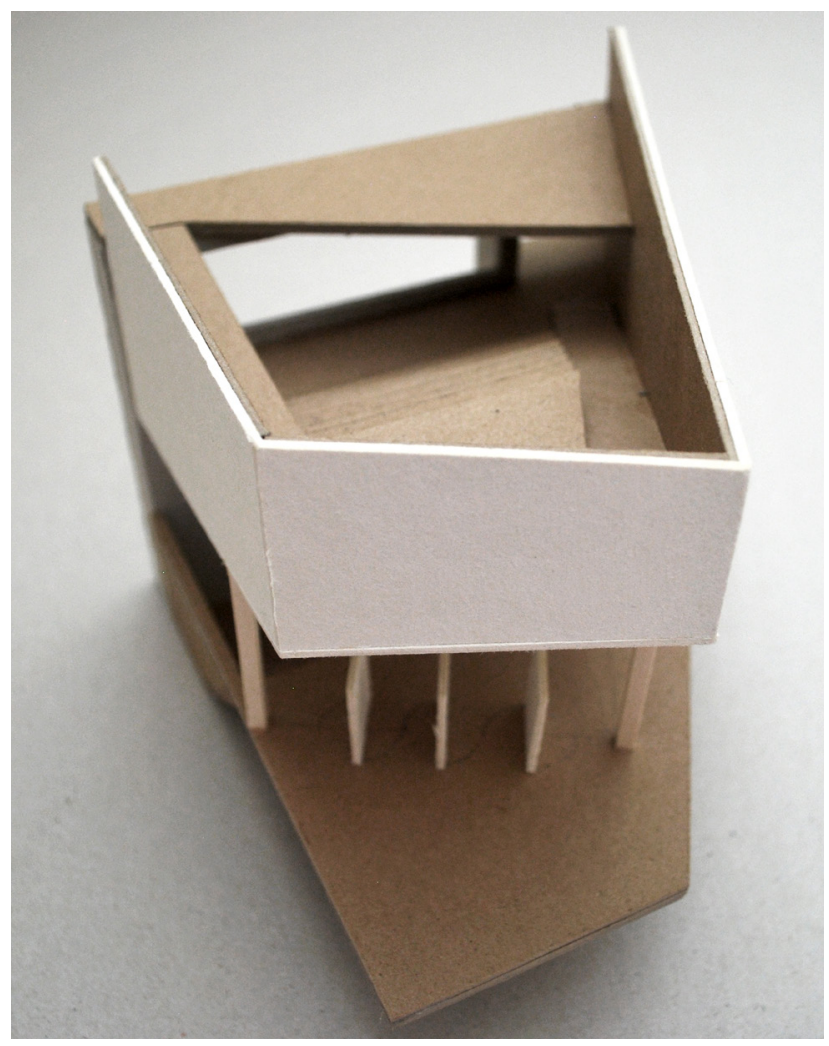

to the exterior. This is done to create a canopied exhibition space that is able to fluidly transition from interior to exterior. Positioned along the circulatory narrative is the second auditorium which faces an aperture opening up into the sound stage. The node created emphasizes a connectivity to the production of work. The space doubles as an impromptu teaching gallery and informal gathering space

FIGURE 55 MODEL

Entrance Exhibition Vignette

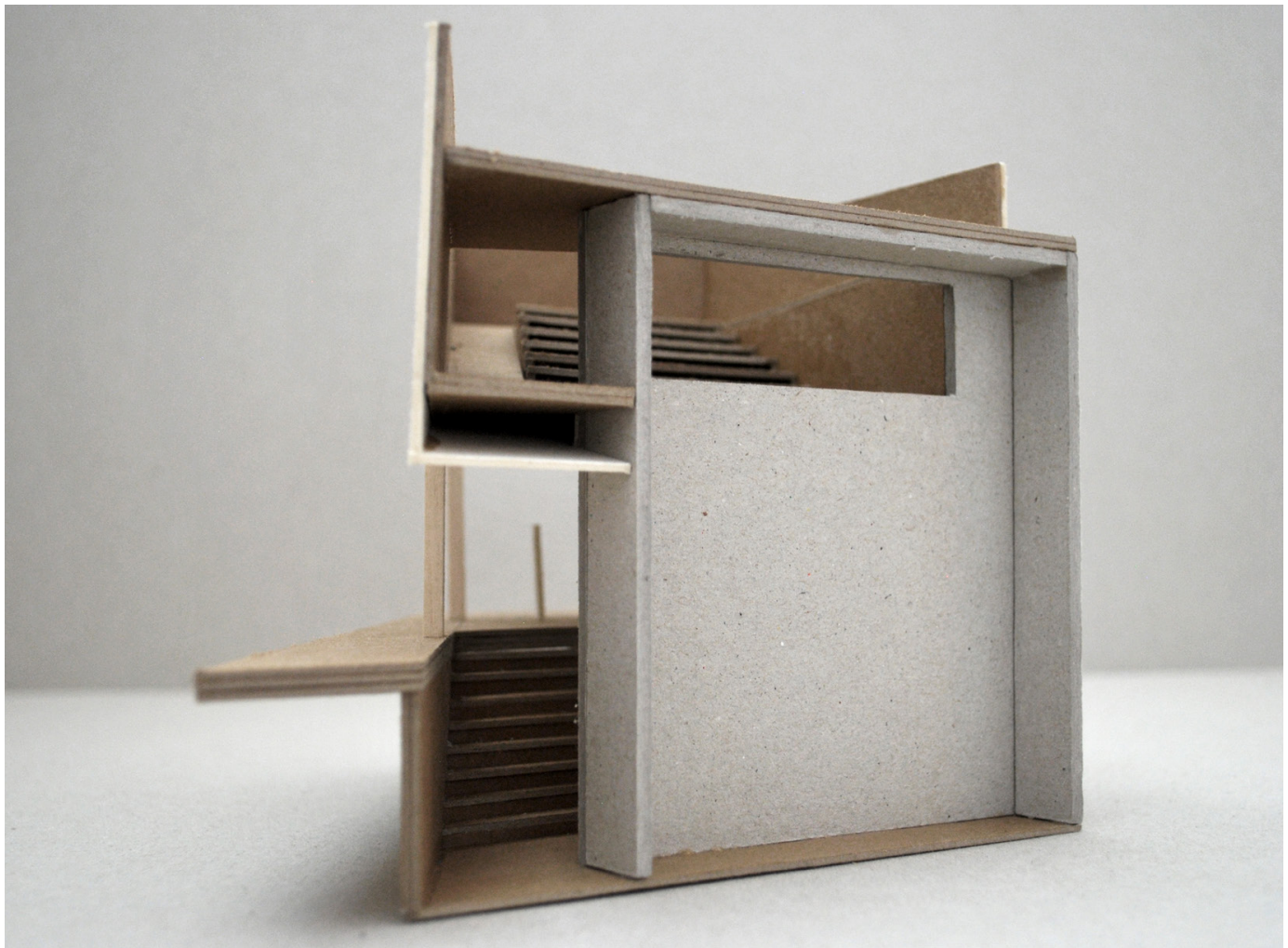




\subsection{MULTI-PURPOSE NEW MEDIA LAB VIGNETTE}

In blowing up the multi-purpose new media lab two primary aesthetic and functional drivers became apparent. Stemming from the upper flow sectional model it became important to maintain overall visibility through the space while allowing the room to become enclosed. This is achieved by floor to ceiling glazing on the exterior with a retractable black out curtain and on the interior wall a folding acoustic partition. The folding acoustic partition acts as a loading access for the space when setting up and continues as a backdrop during filming. Additionally the ability to completely retract allows the space to open up during exhibitions and other moments when total enclosure is not necessary. The room is lined with acoustic dampers to minimize sound infiltration. Finally a $60 \mathrm{~cm}$ overhead track grid is built in allowing users to hang temporary partitions and lighting/sound equipment.

The space is intended to function in the manner of this initial shadow box models, creating a visual buffer exhibiting process. From the exterior the space becomes a focal point and a gateway for exhibiting individuals at work. From the interior the operable acoustic partition creates a buffer zone, filtering passersby's view of the far wall with individuals working on set.
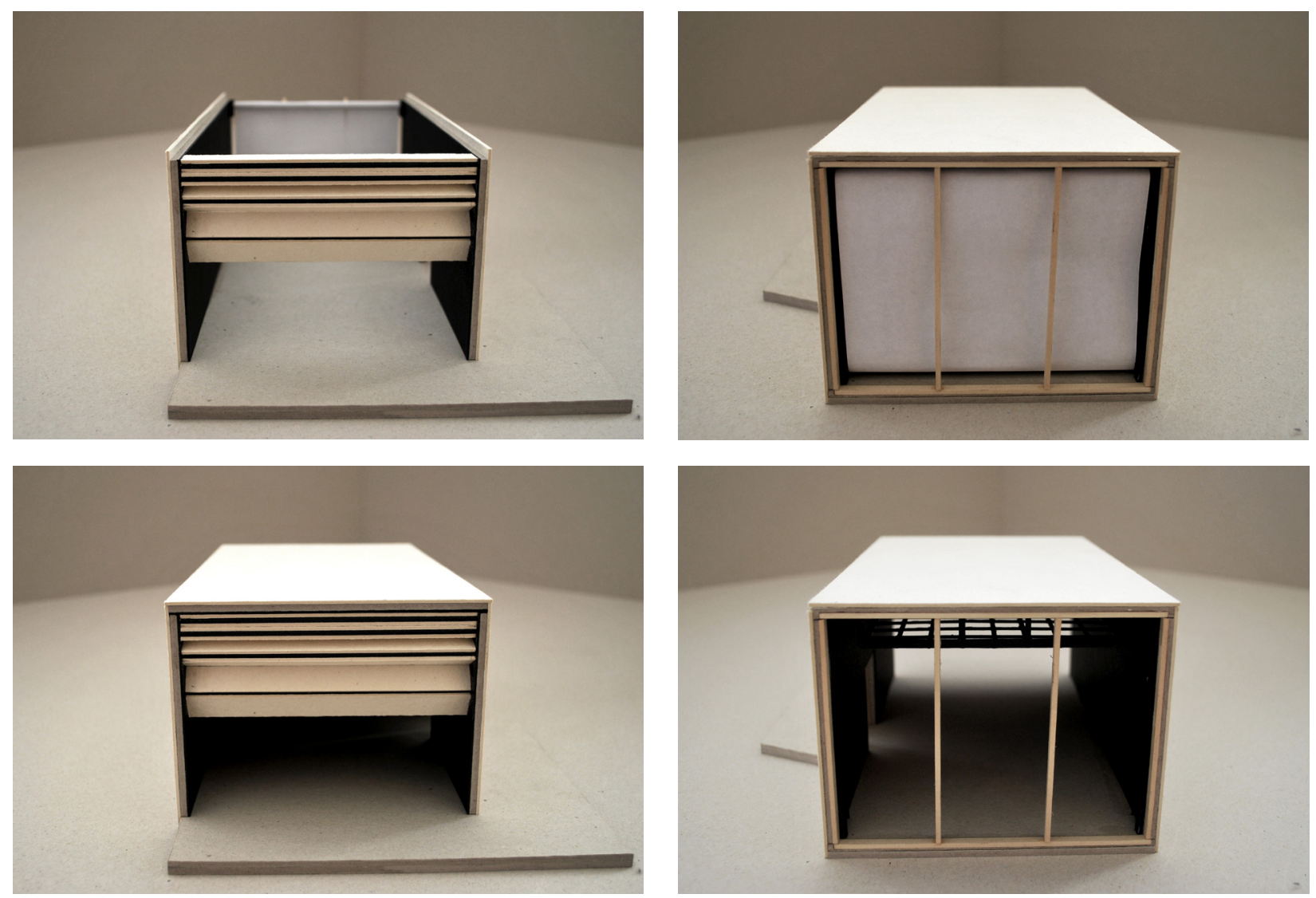

FIGURE 56 MODEL 
FIGURE 57 MODEL Multi-Purpose New Media Lab Vignette
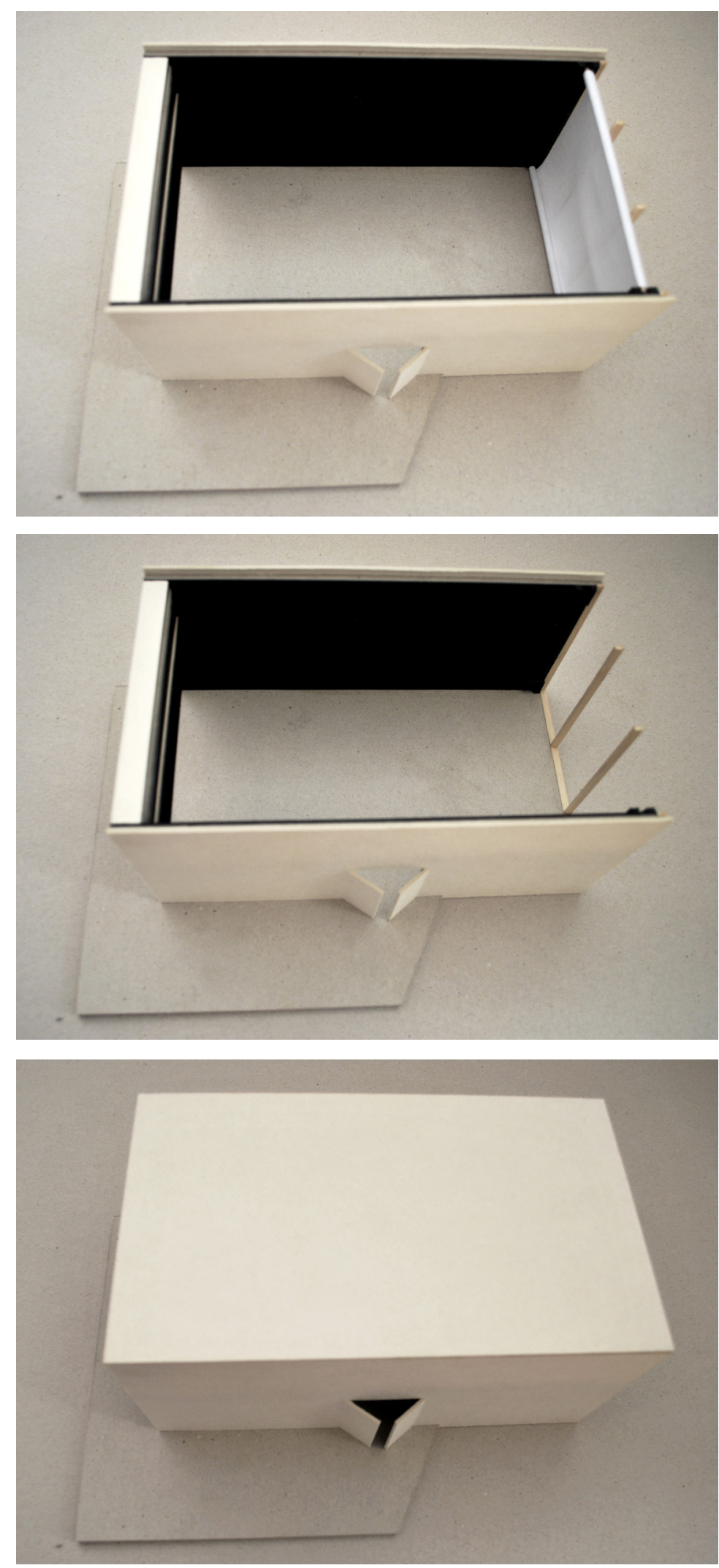


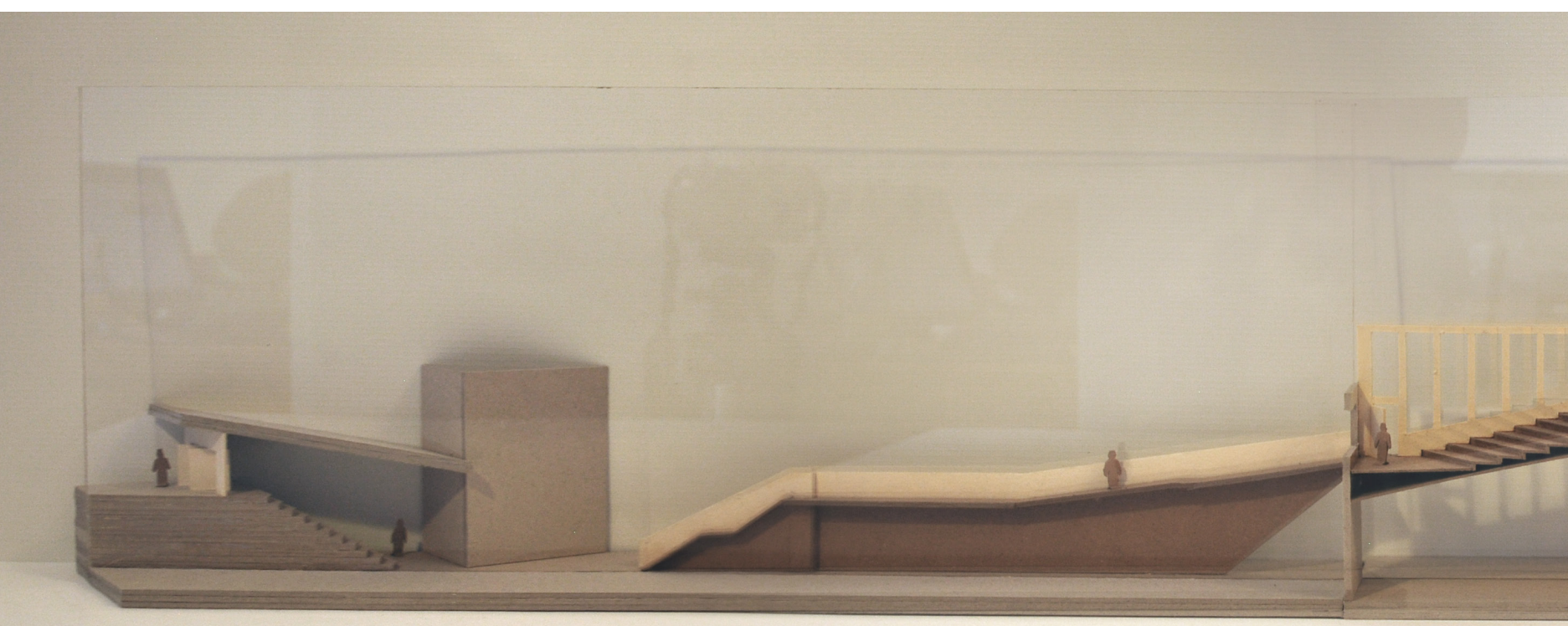

\subsection{UNFOLDED CIRCULATORY NARRATIVE EXPERIMENT}

The previous partial models attempted to recreate an atomized iteration of the circulatory narrative through the building, by beginning to construct key vignettes of moments of significance at a larger scale. This experiment developed a more thorough understanding of the particular qualities of the spaces under question however with a lower level of abstraction the vignettes quickly begin to lose the original intent. Instead of reading as standalone elements, the vignettes began to require all of the work that preceded it.

In the development of stand-alone artifacts for a design which is slowly evolving it becomes important to stress the level of abstraction in an attempt to gain a more accurate essence of the concept under question. The circulatory narrative, a winding path which leads observers through the various stages of production is removed from the container that houses it. In striping the circulation from the building the narrative is not constructed by the work that preceded it, merely utilizing it as a reference to emphasize the journey. A 5m alternating section was drawn through the building, focusing on the observers' relationship to the spatial qualities and the transitions between nodes. Straightening the winding path and allowing the experimental model to alternate between sides of the section line further removes the artifact from the finite building, 


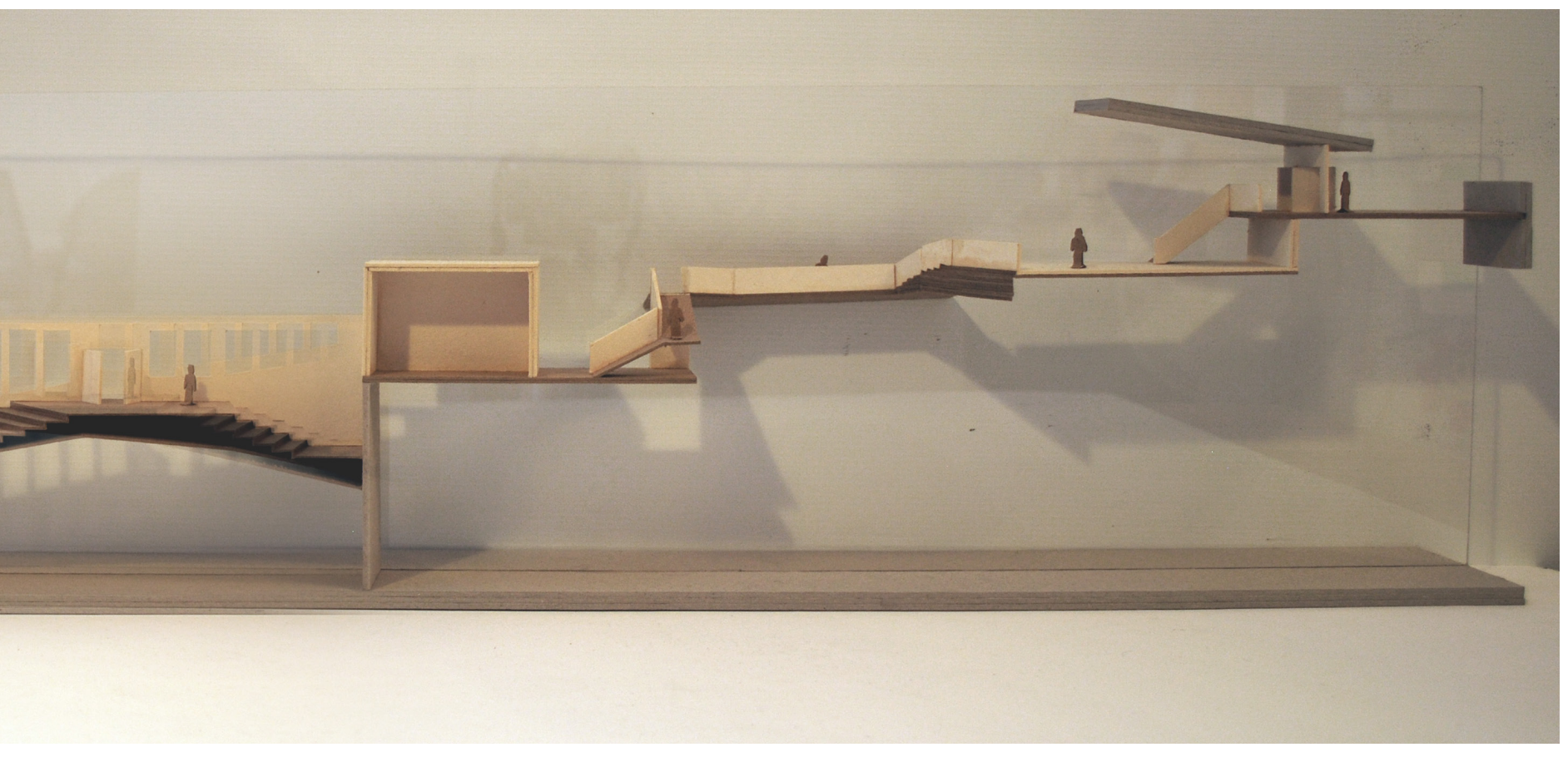

FIGURE 58 MODEL

Unfolded Circulatory Narrative Experiment

The success of the experiment lies in the ability to be interpreted sans preface. Moving forward it becomes pivotal to not simply continue to recreate but with each design experiment to place an emphasis on injecting a new or different perspective or take on the issue at hand. Future iterations must place emphasis on expanding the dialog in a meaningful way or radically reinterpret abstracted space as to not fall into the same pitfall as the previous nodal vignettes or partial sectional model. In challenging the message a more refined understanding of what is trying to be said will emerge. 


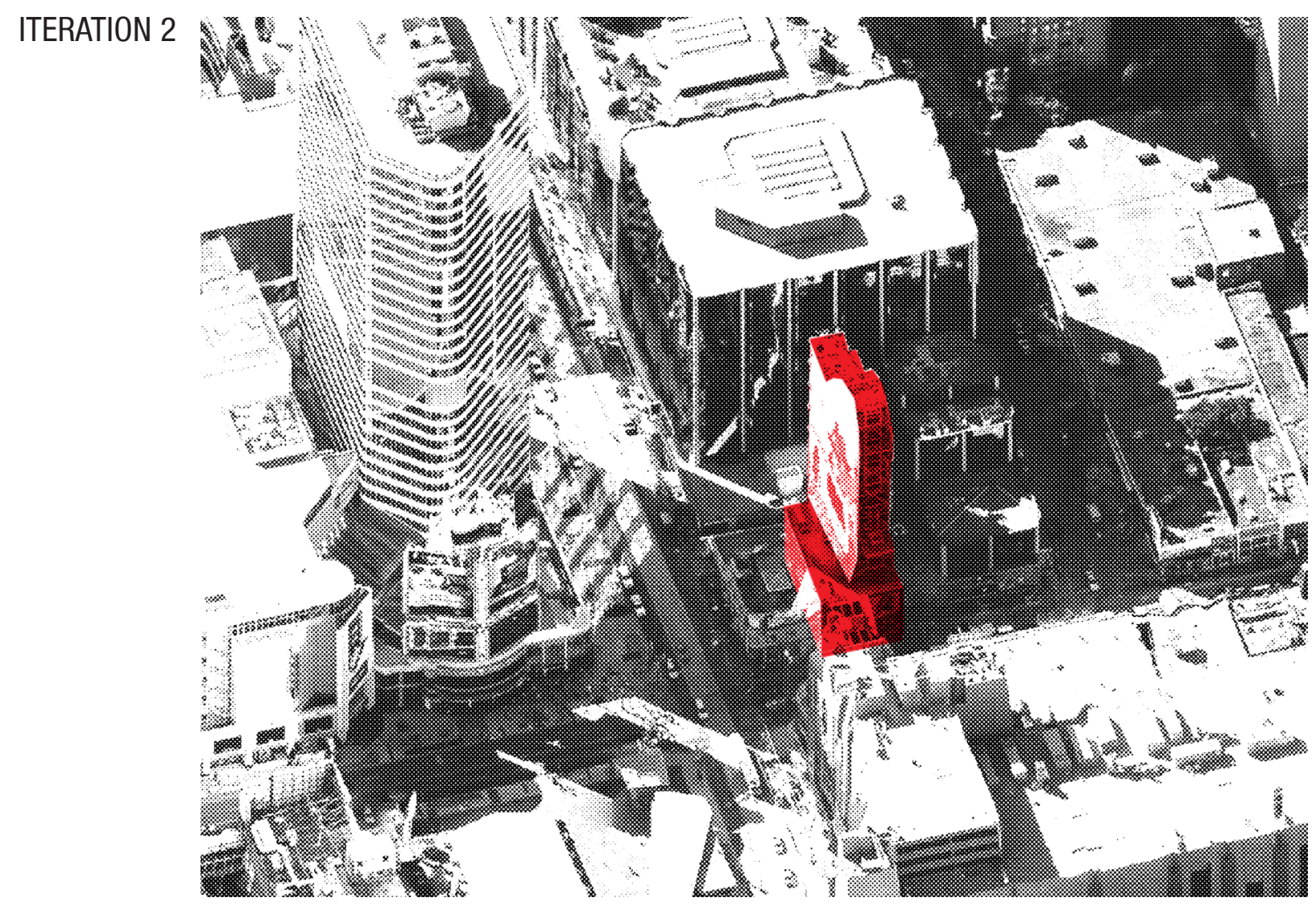

ITERATION 1

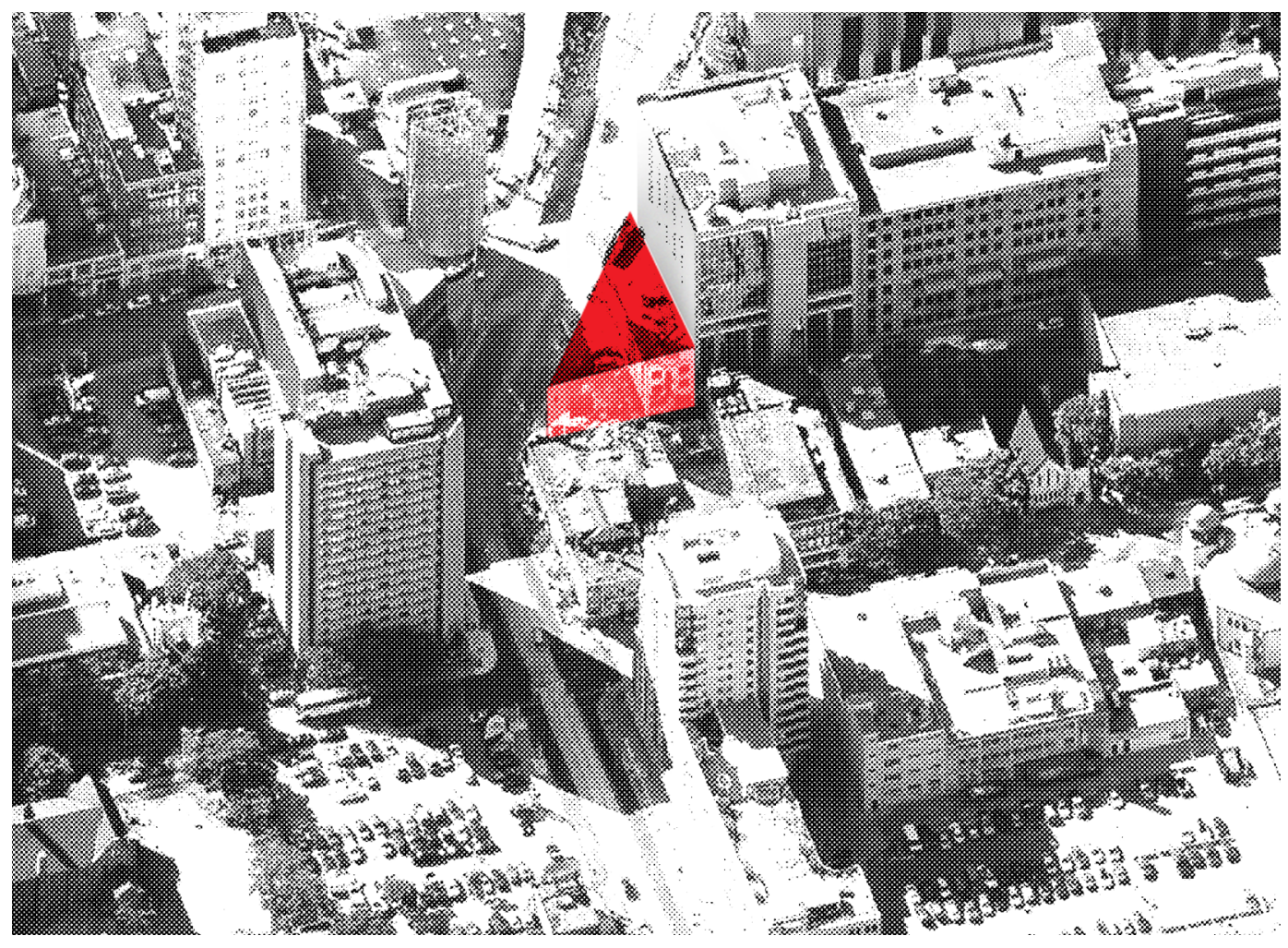

FIGURE 59 MAPPING 


\section{CHAPTER 14 \\ DESIGN 2: VERTICAL NARRATIVE}

\subsection{RE-ANALYZING DUNDAS SQUARE}

The unfolded circulation section, which guides the viewers through the major narrative nodes of the project, acts as an accumulation of the initial conceptual drivers as evolved from the first site conditions. Instead of continuing the discussion through the refinement of said design experiment, it became evident that in altering a fundamental aspect of the previous project a purer understanding of concepts will emerge. The act of changing the foundation upon which the experiments were constructed is an attempt to facet my existing understanding of the concepts and permits the exploration of previously suppressed ideas that ran tangentially in the earlier design project.

Fundamentally the ideas explored were that of the shadow box, or the visual layering of spaces to alter the perceived hierarchy of process and the circulatory narrative as a vessel for constructing an understanding of process. The two ideas were born simultaneously from the site and programme, with novelty derived from ideas of multi-facetedness and the rejection of flatness.

The second site under exploration is 306 Yonge St, located on the northern edge of Dundas Square. Most notably the site houses a large advertisement structure above a presently vacant 2 storey commercial building. Although both sites are located along the edges of Dundas square each contain distinct spatial characteristics. The earlier site at the intersection of Dundas St. E and Victoria St. is triangular, resulting in primarily wedge shaped massings. Additionally with a comparatively larger floor plate, the programme is comfortably contained in approximately 4-6 storeys (depending on the iteration). 306 Yonge St. conversely is a substantially narrower site with a focus on verticality. Proposals on this site are not afforded with the same liberal use of space experienced on the first site. The two proportionally different sites will hopefully produce varied interpretations of the same concepts. 


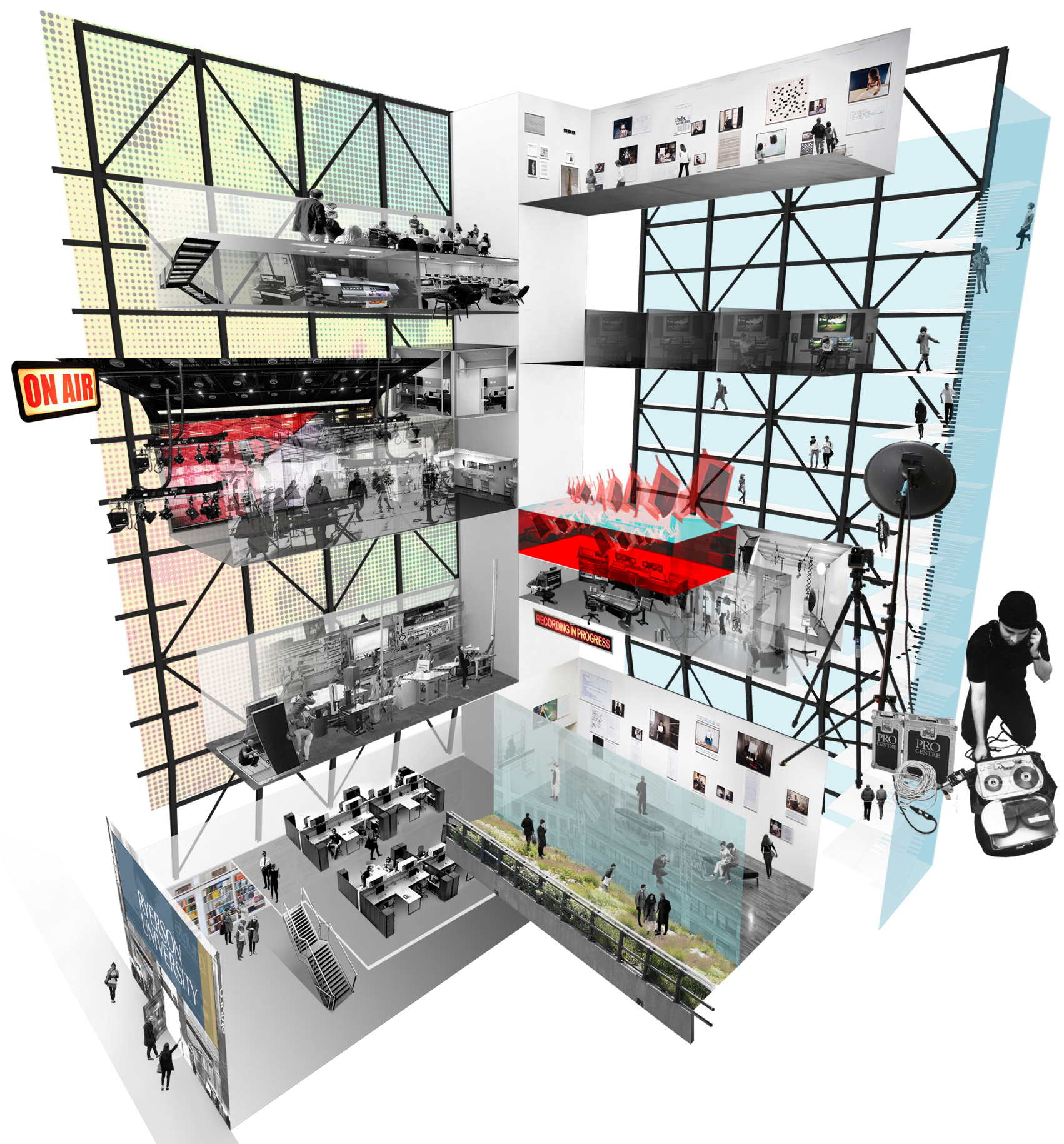

FIGURE 60 COLLAGE

Visual Narrative 


\subsection{FIRST DESIGN EXPERIMENT}

This visual experiment documenting the designs conceptual evolution explicitly exaggerates the production process. The artifact itself creates a sectional split through the building and anchoring the two halves along the primary circulation route. The programme of the building is grouped for the stages of media production beginning with the commercial entrance and support office spaces in the bottom building. The tower itself is bookended with two exhibition spaces referencing inspiration and presentation, with raw production in the bottom half and more finalized post production in the upper floors. The experiment, in order to emphasize the isolation of each node, alternates the spaces from north to south façade. Additionally the emphasis is given to the interior conditions over the exterior conditions as would conventionally be granted from exterior views.

In adapting with the narrower floor plate and generally tighter conditions the circulatory narrative is forced to evolve in scope from the previous design. As opposed to winding scheme which directed the observer through a series of programmatic nodes, the narrative is constructed as a series of isolated environments stacked upon one another. Each environment behaves as a node, filtering the production experience as the observer moves through the spaces. While previously the transition is continuous, with each node unfolding before the observer, this scheme is very abrupt, understanding that individual spaces inspire a distinct and isolated understanding of each step.

The shadow box additionally has been handled differently. The previous scheme saw production spaces given large aperture's for observers to look peer into the production spaces. The two primary facades offer two iterations. The southern façade, facing Dundas square, retains its existing connection to the visual jungle of the square itself. A large permeable screen runs along the entire façade. The spaces behind the screen offer various degrees of transparency based on the production undertaken within them. This creates a dynamic interplay between building occupants and advertisements as the location of advertisements is not fixed allowing transparent production spaces such as the workshop to be displayed at the same time as promotional material. Environments such as the computer lab or multi-purpose studio can fluidly transition between displaying the interior spaces or artifacts. 
The northern façade on the underhand produces a juxtaposing condition, where building occupants are permanently on display. Circulation at this point can be interpreted as the shadowbox with the $2 \mathrm{~m}$ wide corridor buffering the production spaces. With the corridor remaining relatively shallow and incorporating unobstructed views to the interior environments when possible, producers are always at the forefront.

The major downfall of the collage inspired experiential section in this instance is the suppression of the narrowness of the building combined with a highly sectional attitude in spatial design. Similar to the previous iterations it is crucial to understand the spaces that are created physically. While it can be argued that the iterations on the previous scheme were more plan based, their effectiveness is primarily comprehended through the exploded models. To fully grasp the ramifications of working with such a narrow and vertically dominant proposal, a more tangible form of expression should be employed. However the key note moving forward is to develop means of communication that is not a mere translation of the image into a model and to understand, similarly to the unfolded experiential circulation model, that the artifact must embody a previously unseen facet of the project. The next iteration must elaborate upon a knowledge derived from this iteration. 


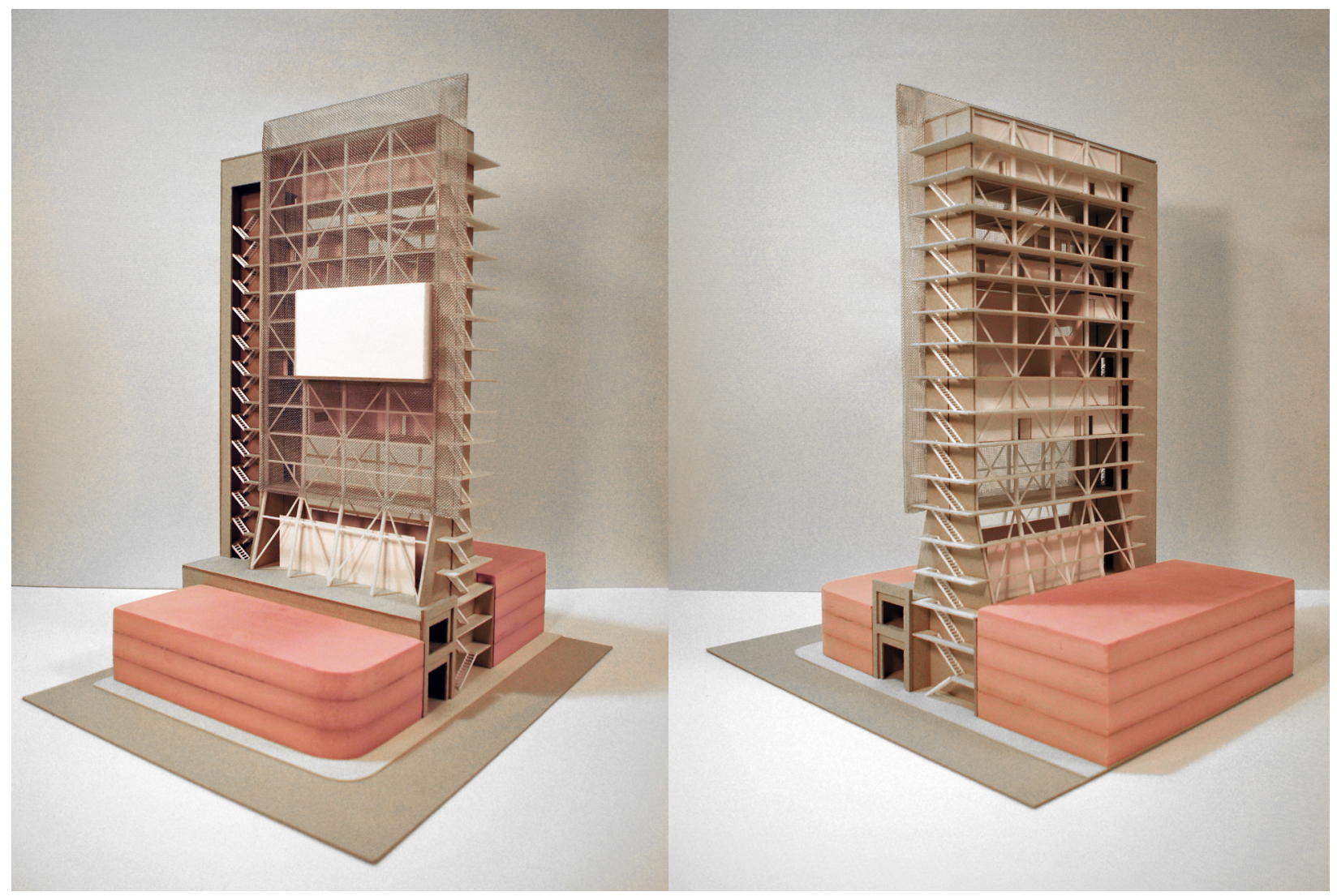

FIGURE 61 EXPERIMENT

Exterior Views of Model

\subsection{SECOND DESIGN EXPERIMENT}

The first component of the second design experiment is the creation of a physical translation of the previous iteration. In doing so several repressed facets of the building were uncovered, in particular the duality between the north and south façade and the overall narrowness of the structure. When closed the model recontextualizes the work through the use of scale, drawing relationships to the adjacent buildings and street, and emphasizing the correlation between the building and the main circulation path. In creating both the interior and exterior conditions the notion of the shadow box becomes more clearly evident. The north façade creates a distinct circulation corridor that is previously obscured; while the southern façade contains a large screen which allows creates a filtered view of the interior space. Three major components of the design expressed in the model is the circulation, the tower structure and the base building. 

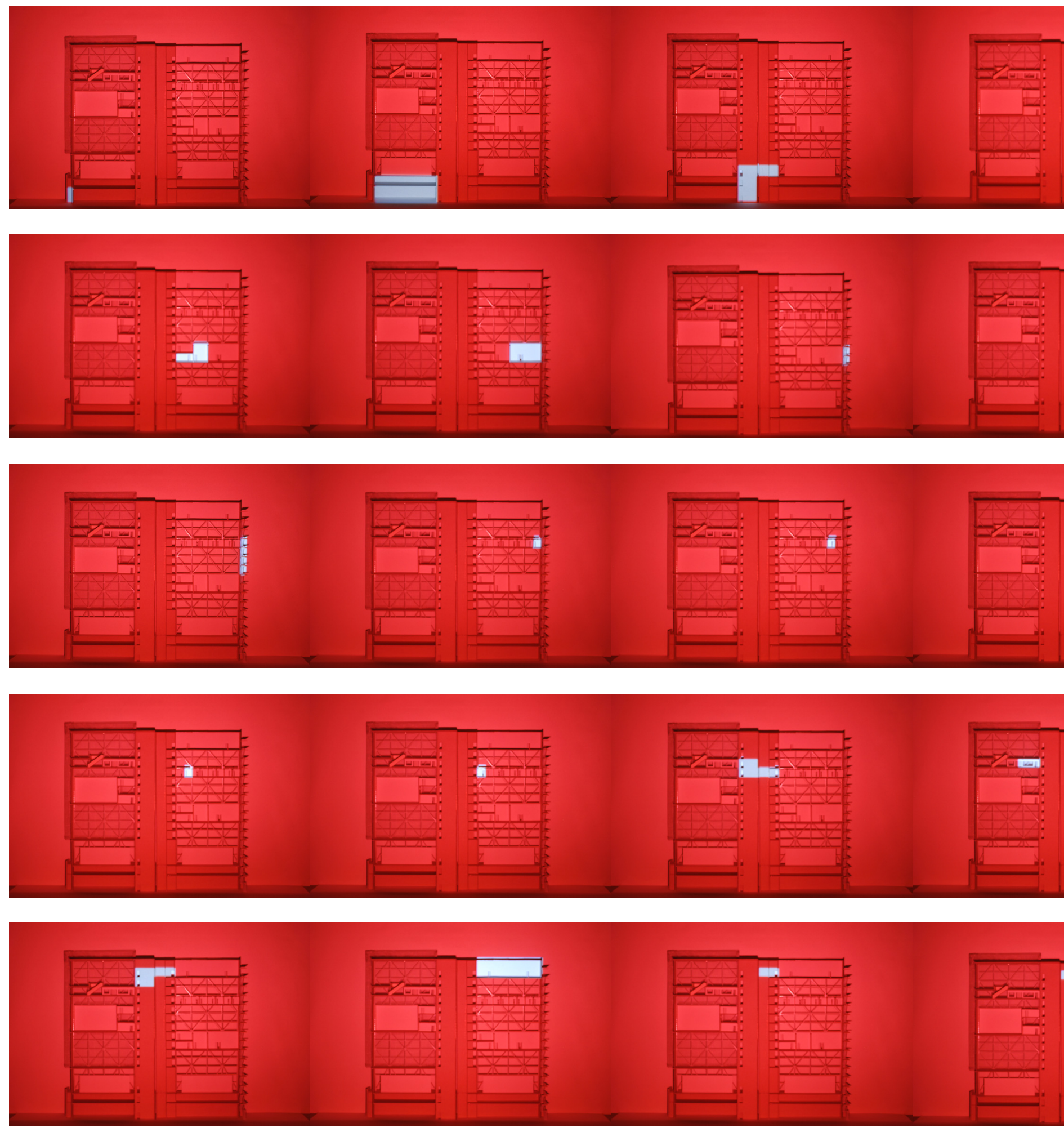


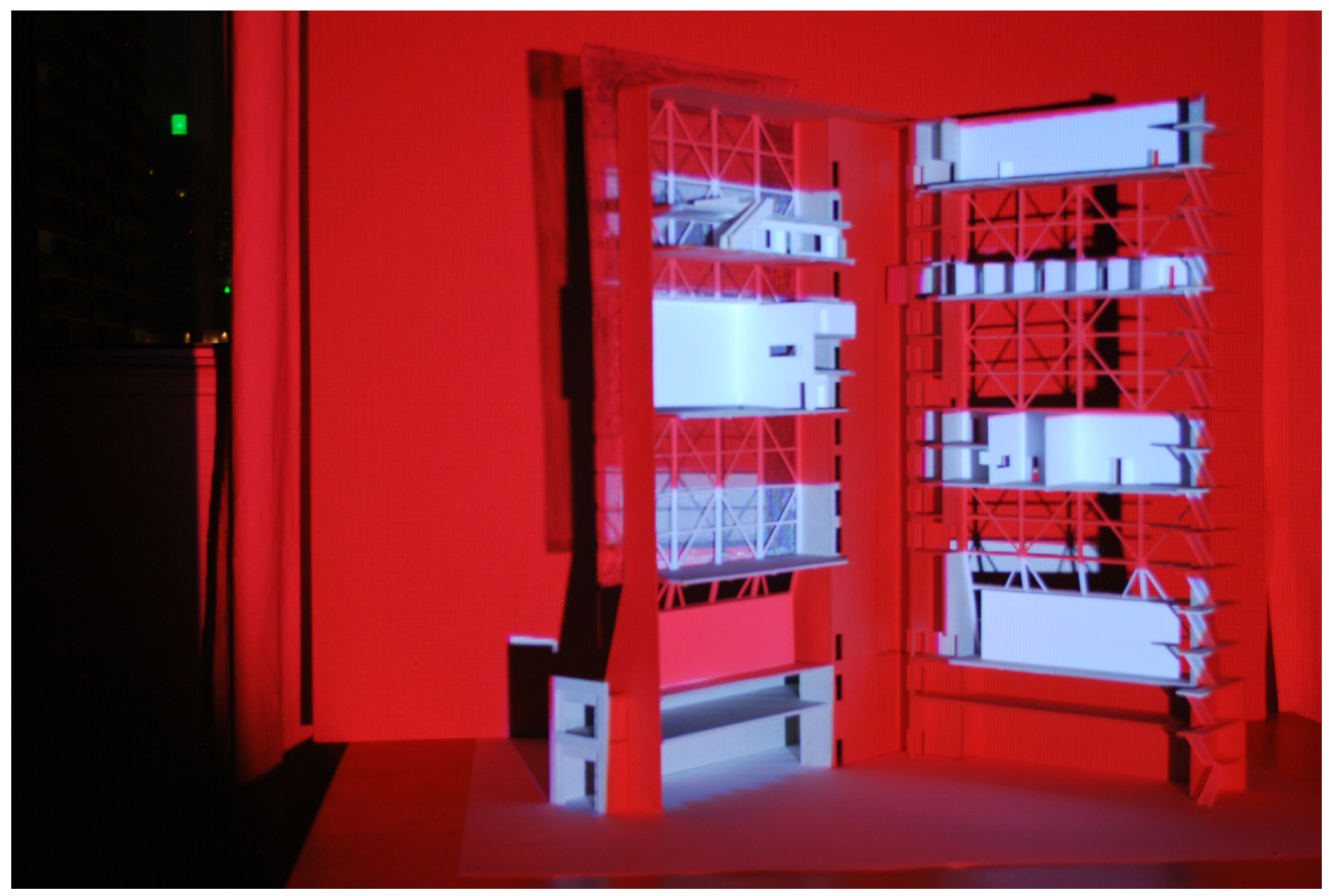

FIGURE 64 EXPERIMENT

Projection Testing on top of Model

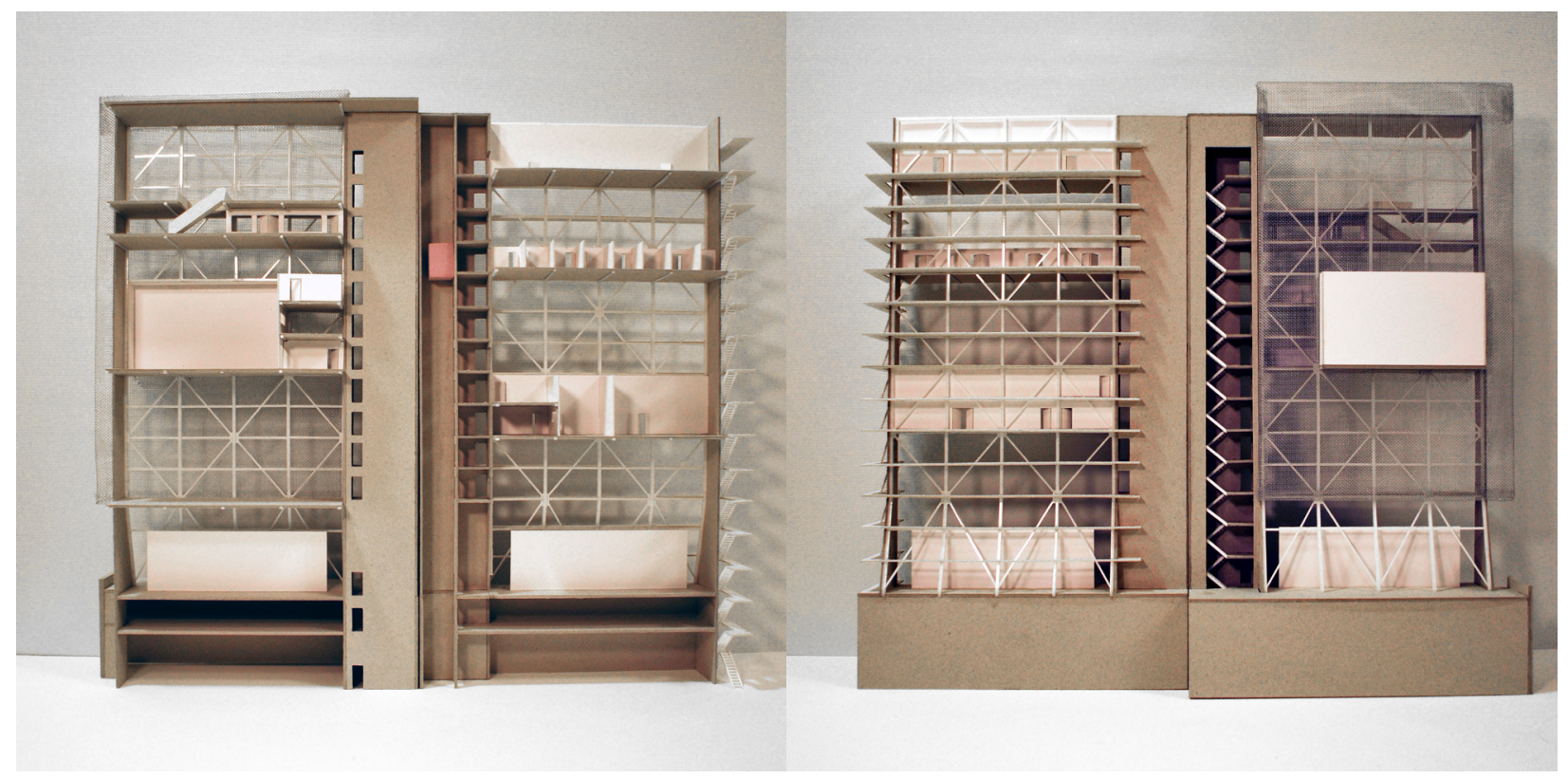

FIGURE 63 EXPERIMENT 
In making a more tangible artifact, the intangible atmosphere created through the use of collage is lost. The model however is conceived in a way that it would open up, similar to the image to allow for postproduction to reintroduce atmosphere. Similar to the unfolded circulation sectional model, the circulatory narrative is investigated. A carefully crafted building walk through is created with the use of a projector, highlighting the individual spaces in time with an audio soundtrack. Sound constructs the atmosphere of the spaces creating a real time circulatory narrative. Each space utilizes an aggregation of distinct sounds which embody the observers travel through the spaces. Each space is broken through the entry and exit of vertical circulation, characterized by a hollow ascent up flights of stairs. This is done to create a buffer between the spaces and emphasizes the various spatial qualities presented within the building as one ascends through the various stages of production. Ultimately the observer ends up in the upper gallery space, where they take the elevator to the ground floor, and their exit, completing their simulation of the design.

The overlay of information provides a unique break from the monotony of purely visual information and creates a certain amount of abstracted distance which calls the observer to reconstruct an atmosphere similar to that of the collage in the previous iteration. The abstracted distancing is the same technique utilized in the sectional parti model (which asks the viewer to construct the extents that have been omitted by the designer) and the unfolded circulation sectional model (which removes peripheral information and allows the observer to focus solely on the circulation as it weaves through the building). The process of restricting access to information derives a purer understanding of a particular facet of the work, highlighting an essence of a project that is lost through conventional spatial translations such as working drawings or renderings. 


\title{
CHAPTER 15
}

\section{GONCLUSION}

\section{"Maybe, like the title (which we are no longer sure about) this installation is about the anxiety produced by the blankness and the terrifying struggle with the terrifying question, 'Are you sure this is enough?' Or is this too much?}

\author{
Andrew Atwood in 3 Preface (Atwood \& Neimark 2015) (39)
}

How does one begin to conclude something that can does not have a definitive end? A year and a half ago I approached the members of my committee with a dissatisfaction on the way that architecture is presently communicated. The rapid turnover and constant update culture present in our digital era, has left an insatiable void in regards to how content is created and disseminated. Since those initial meetings I have been attempting to pinpoint exactly where my dissatisfaction occurs and define what issues are plaguing the architectural artifact. As much as this thesis attempts to capture the present condition of artifacts, the issues facing them and their possible remedies, this thesis has evolved into a quasi-diary. Each essay, model and experiment creates a snapshot of a particular mindset present at every permutation of my outlook since those initial meetings.

This final essay, tucked into the back of the thesis is merely another pause for reflection experienced throughout the aggregation of this thesis. Instead of informing the next design permutation or exploration the intent of this essay is to capture the underlying messages of the book and to distill the cumulative evolution I have experienced throughout this process. As opposed to being interpreted as the end of the journey this essay is merely the framework to be massaged in all work that proceeds it. With every pause is a recalibration of trajectory, or an assessment of the cumulative lessons learned over the course of this journey.

From this thesis emerge two fundamental threads of discourse that are tightly woven into my psyche yet begin at disparate spectrums. The first being that of a criticism on artifacts and the second on the creation of artifacts. 
There will forever remain a disconnect between the artifacts architects produce to communicate architecture and the structures or ideas they are intended to express. The blurred zone between idea and artifact is unavoidable, as it should be. The grey area, or this space of translation is where the fruits of new and interesting directions for architectures future emerge. In constantly challenging and adapting the ways that we communicate our ideas architects, are able to pursue the essence of their concept without the limitations of convention.

As technology evolves and the spheres of information dissemination begin to converge, now is a crucial point to become critical with how these factors are beginning to influence architecture. How is the seemingly endless flood of images afforded to us via the internet changing the ways that we design? Is architecture a handful of money-shot renderings creating idealised moments captioned with generic copy and paste, or buried in books on shelves? Or have we as architects decided that mechanical reproduction is unable to capture the essence of architecture and that it can only be experienced?

I believe that artifacts, whether they are essays or buildings they are merely a screen for architecture. Architecture is the discussion and as long as someone tries to capture the associated mysticism our artifacts will continue to speak. As the ways in which our conversations evolve, our artifacts must continue to evolve. Convention is conventional and by adapting our methods of communication to the current modes of dissemination designers are able to battle the flatness of traditional two dimensional mediums. This attitude is emphatic of the shift to unearthing other aspects such as space and time lost in a photograph for example.

The succession of the image is the challenging of conventional means of expression in regards to the architectural artifact. In conventions place, focusing on the possibilities afforded via technological advances to use a variety of mediums such as models, sounds and projections in the creation of spatial narratives. The image has progressed and disseminated architecture to this point and it is important to continuously ask what is next. The second design employed the use of sound and projection to develop a spatial narrative that was only hinted at with the sectional model. By combining the conventional with the more abstracted methods a temporal atmosphere emerges, a quality lost that can only be alluded to in renderings and orthographic projections.

Embedded within the delicate balance of narrative weight and the flatness that occurs when artifacts are stripped of it, is the importance of abstraction. Equally, if not more important to the information presented, is the information that is omitted from artifacts. Each of the design experiments experience a critical moment 
where the observer is asked to complete the artifact. In the case of the sectional parti model the section cuts leave a vast amount of information uncertain and in the eyes of the observer, additionally both the unfolded sectional model and the audio based narrative projection place the atmospheric qualities of the narrative to the forefront.

The second fundamental aspect of the thesis is the process from which the project came into fruition. The iterative design approach places an emphasis on the creation of a systematic process of creation and analysis. Equally important to each step of creation is the moment of reflection, producing a constantly oscillating trajectory for the work. Each experiment attempted to express a particular concept or series of concepts that were derived from the work that preceded it. Without a focus on an end goal the work grew organically and at times in a highly fragmented pattern, allowing the underlying concepts to evolve as my understanding of the ideas permutated. In not rigidly focusing attention onto making the thesis fit into a tidy container I am able to wholly embrace a particular attitude and truly experiment.

In assessing the strengths and weaknesses of each attempt I was able to document a linear progression of inherently fragmented ideas. Every moment during the course of the project where I had a clear understanding of what comes next, a certain unease emerges. By constantly suppressing the desire to remain conventional was the thesis able to embody the form it takes. The uncertainty of destination allows for a focus on the journey, and deadlines often result in a break. The journey at this point abruptly stops but leaves the impression of all of the stops along the way.

This thesis is accumulation of all the stops along the way. The inherent quality of a multifaceted work is that there will always be more facets to expose. The thesis has allowed me to create numerous artifacts that can be understood in isolation, or woven together under a self-curated trajectory. No single element should require an entire body of work to understand, and in the observers curation is able communicate even more. Each artifact is merely a screen for the conversation you wish to have about architecture. 


\section{APPENDIX I}

\section{DESIGN ITERATION 1 DRAWINGS}

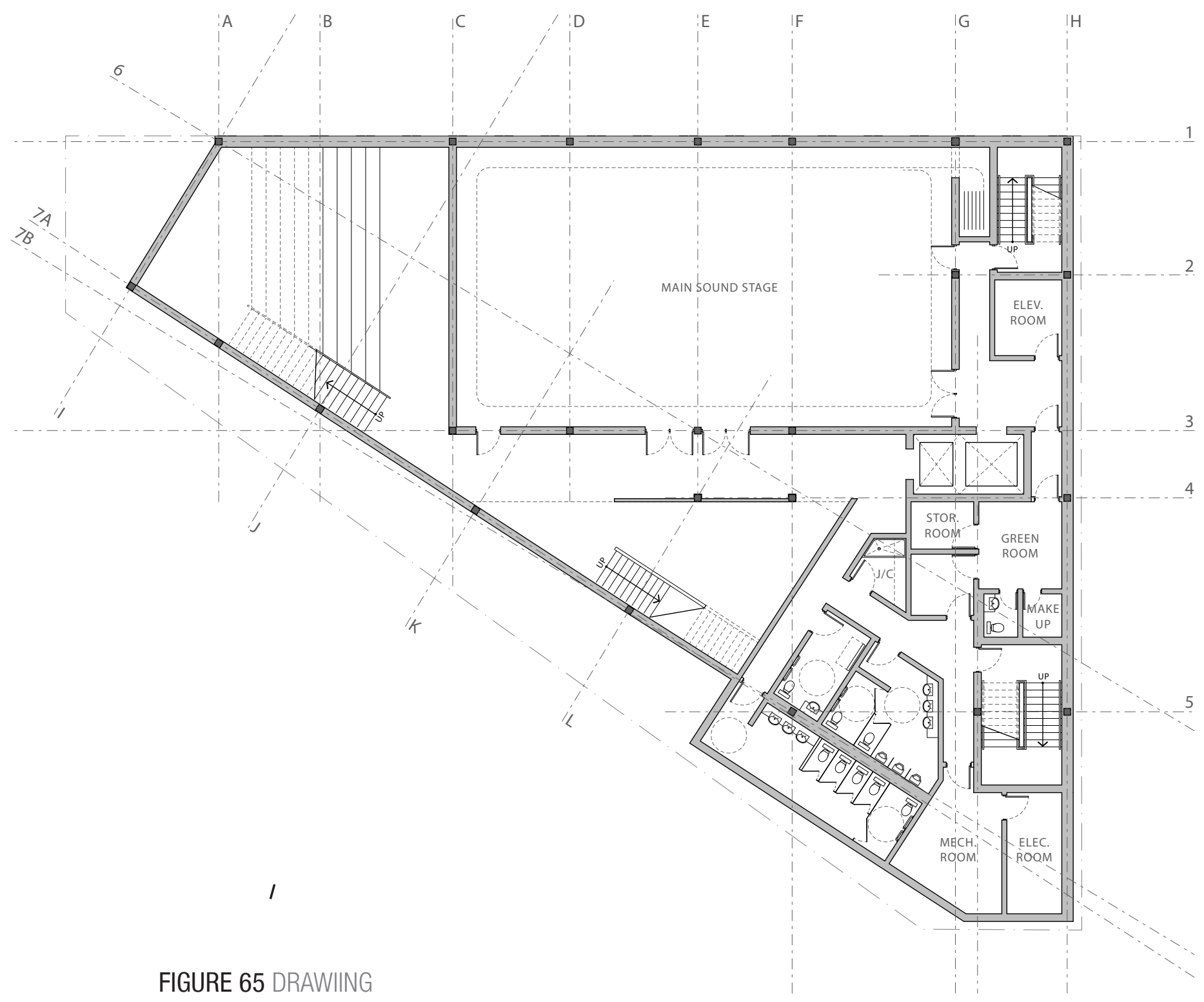

Design Iteration 1 Basement Plan

\N 1:250 


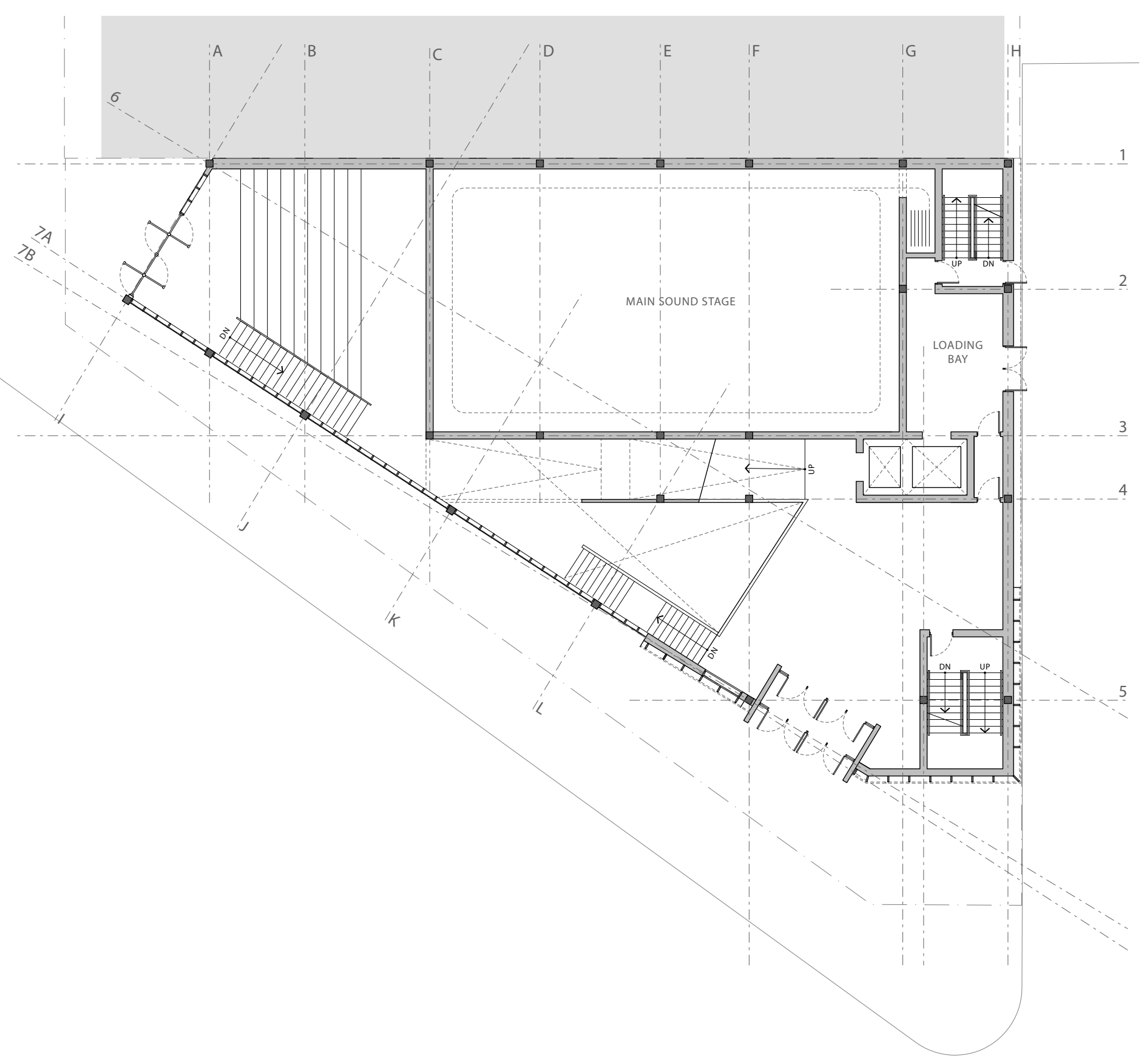

FIGURE 66 DRAWIING

Design Iteration 1 Ground Floor Plan

$\Lambda \mathbf{N} 1: 250$ 


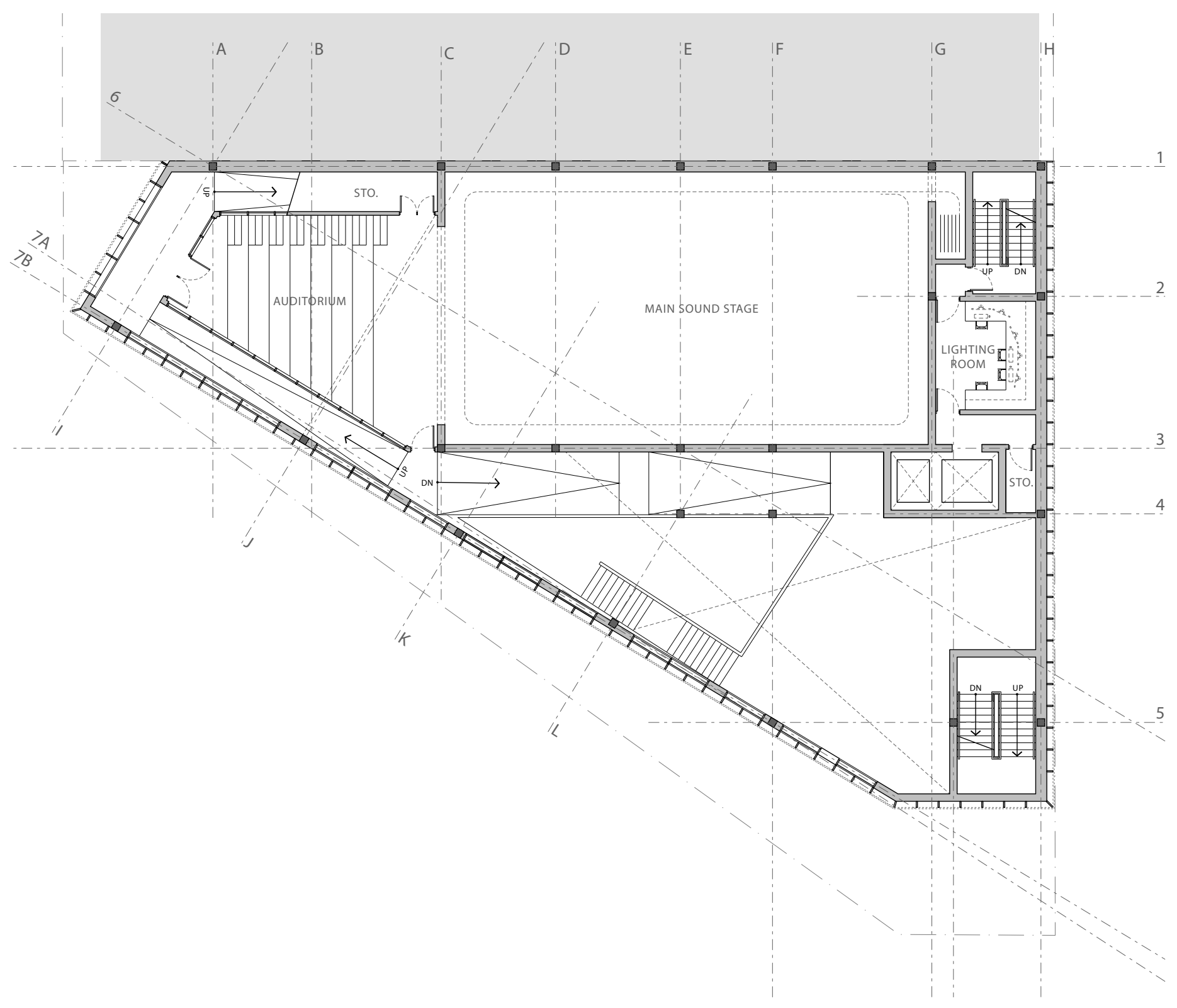

FIGURE 67 DRAWIING

Design Iteration 1 Second Floor Plan

$\bigwedge \mathbf{N} 1: 250$ 


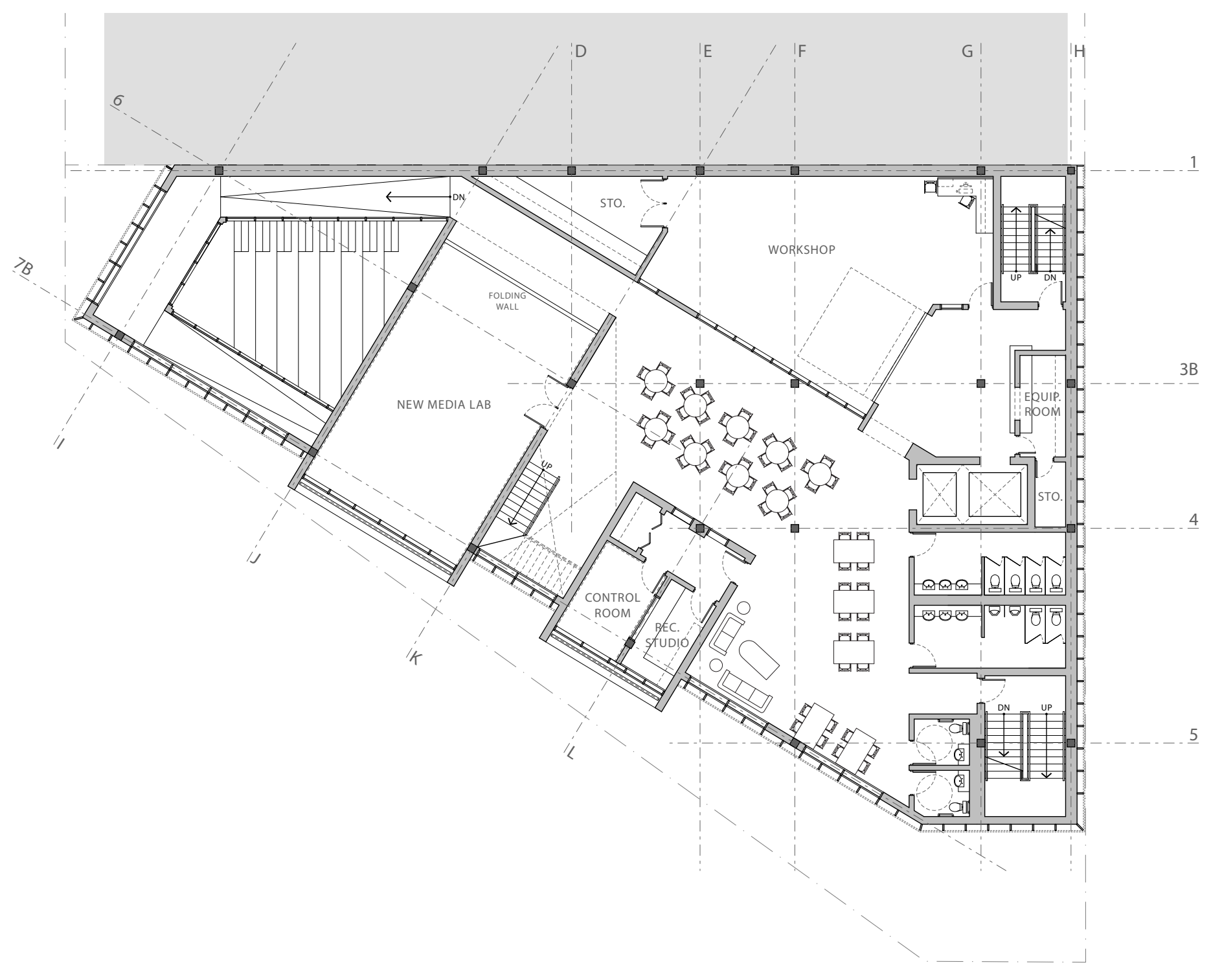

FIGURE 68 DRAWIING

Design Iteration 1 Third Floor Plan

$\bigwedge \mathbf{N} 1: 250$ 


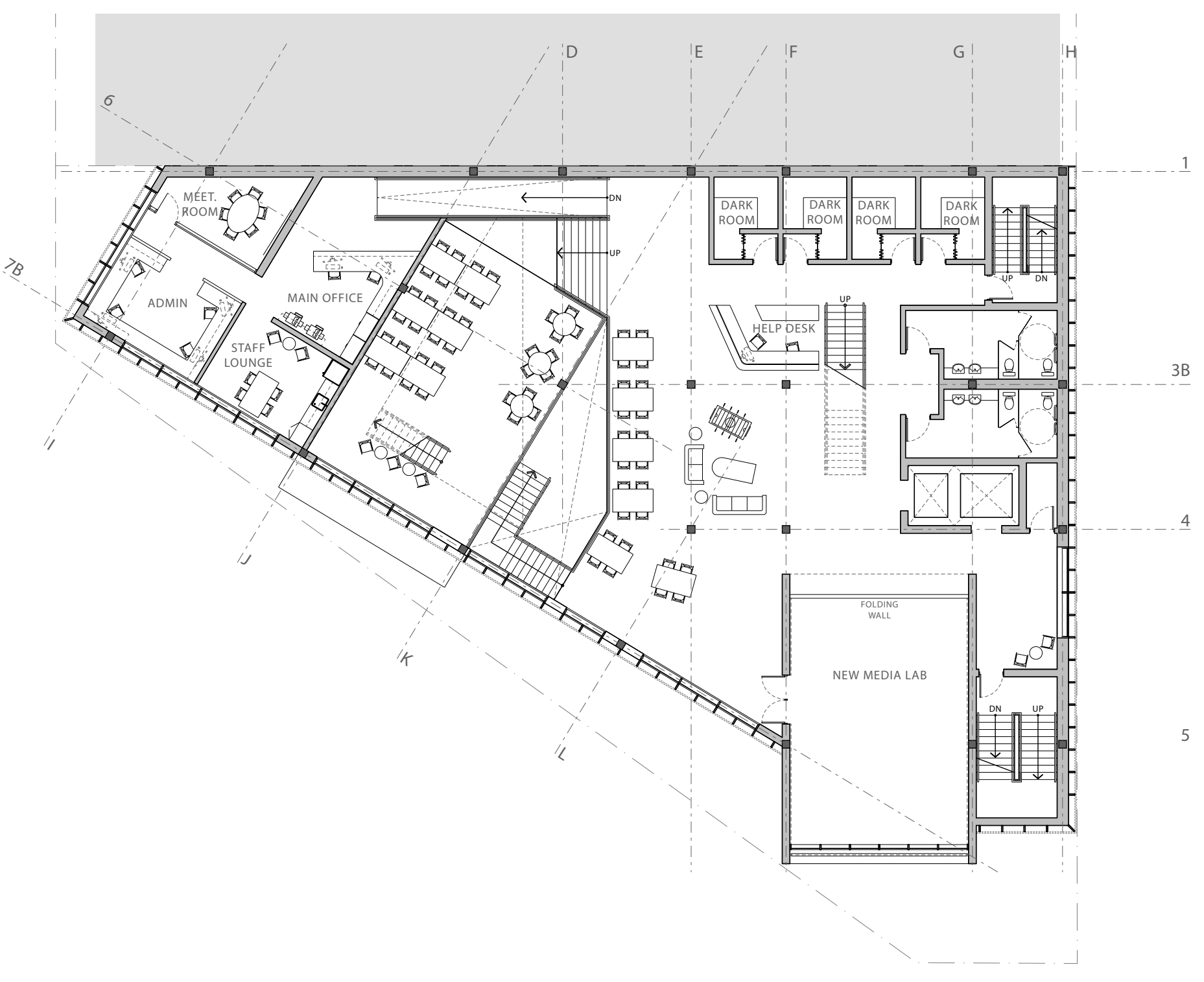

FIGURE 69 DRAWIING

Design Iteration 1 Fourth Floor Plan

$\bigwedge \mathbf{N} 1: 250$ 


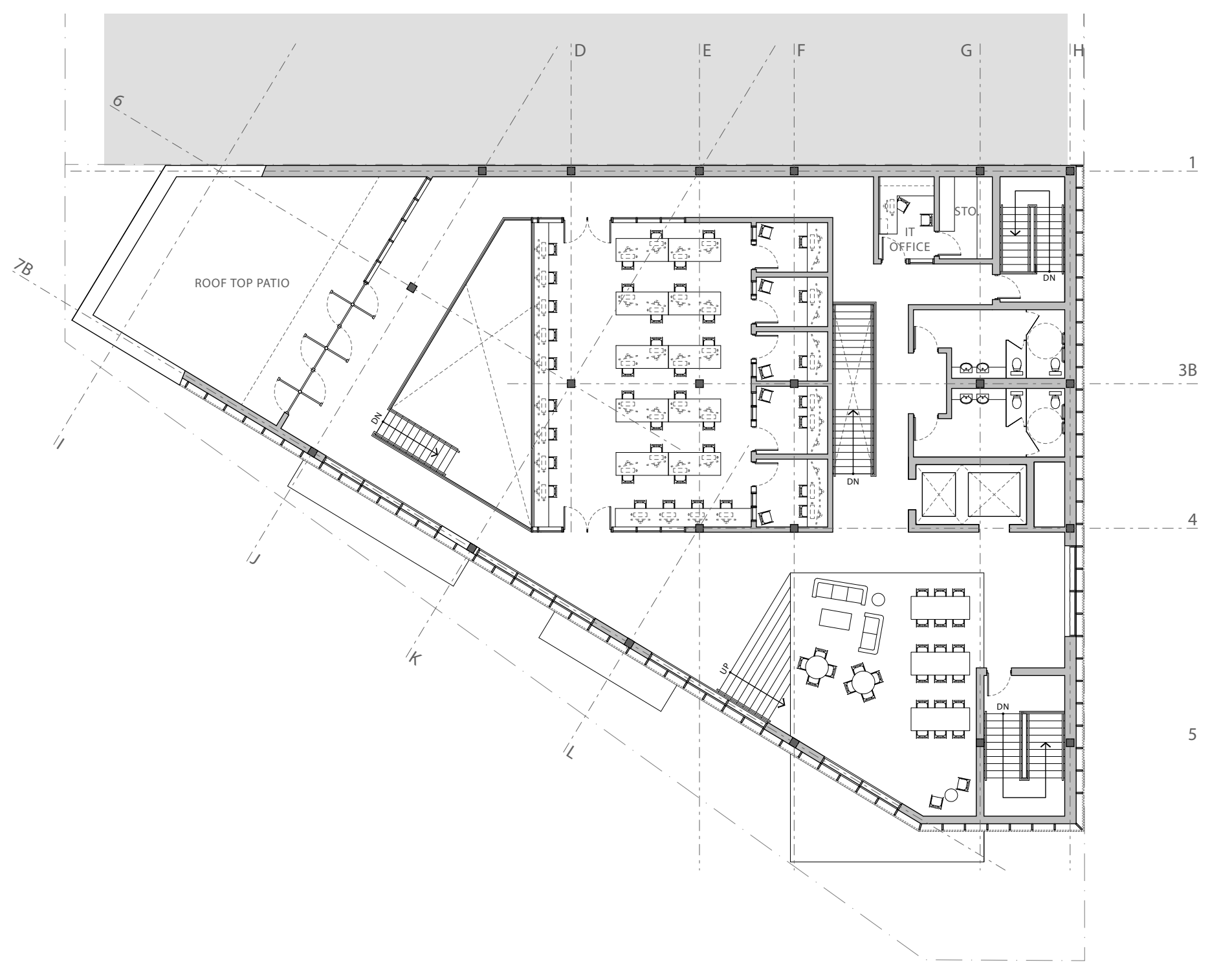

FIGURE 70 DRAWIING

Design Iteration 1 Fifth Floor Plan

$\wedge \mathbf{N} 1: 250$ 
APPENDIX II

SOUND STAGE / STUDIO PRECEDENTS

SPACE LAYOUT AND TECHNICAL DRAWINGS
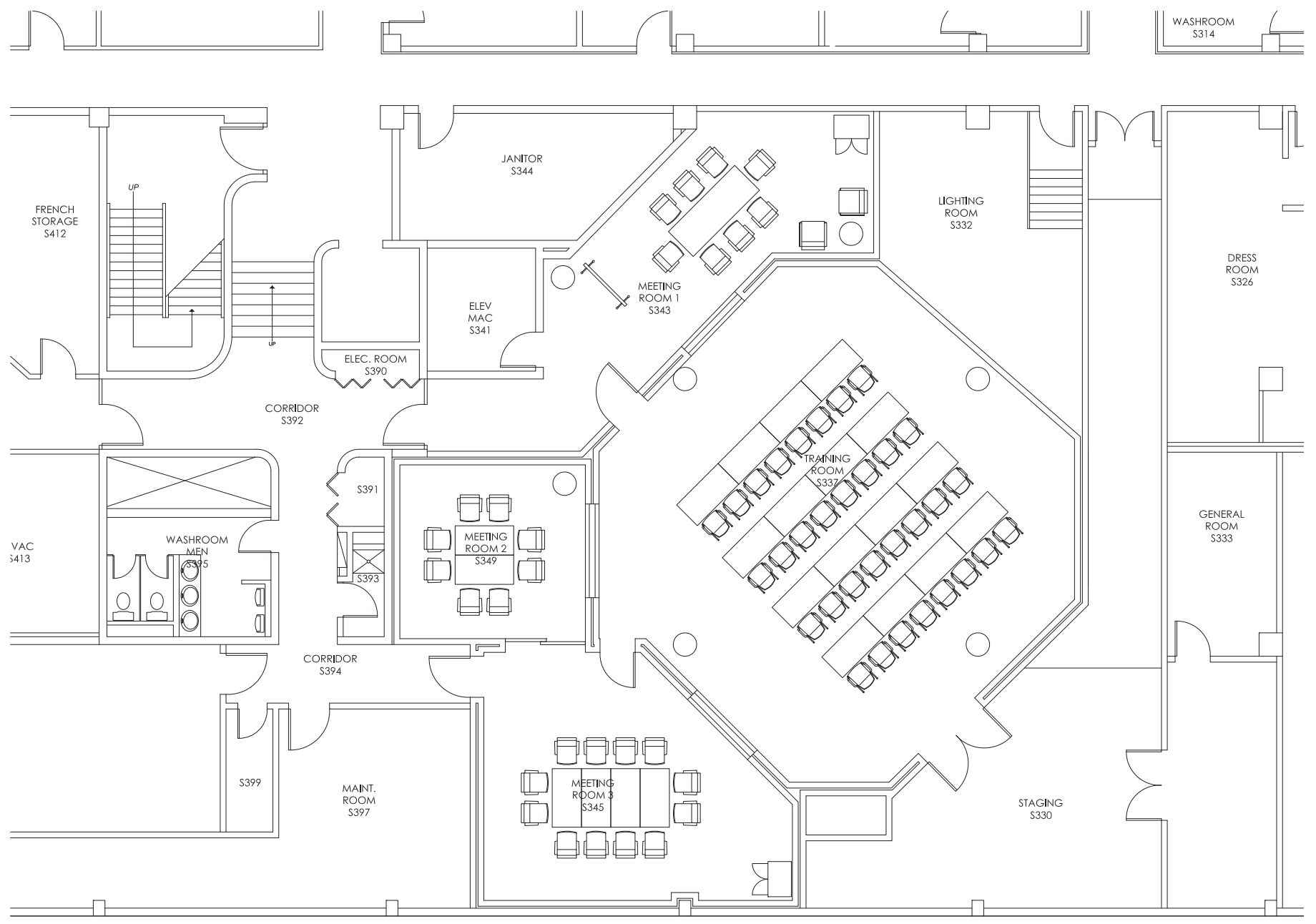

FIGURE 71 REFERENCE DRAWING

CBC Headquarters, Toronto 


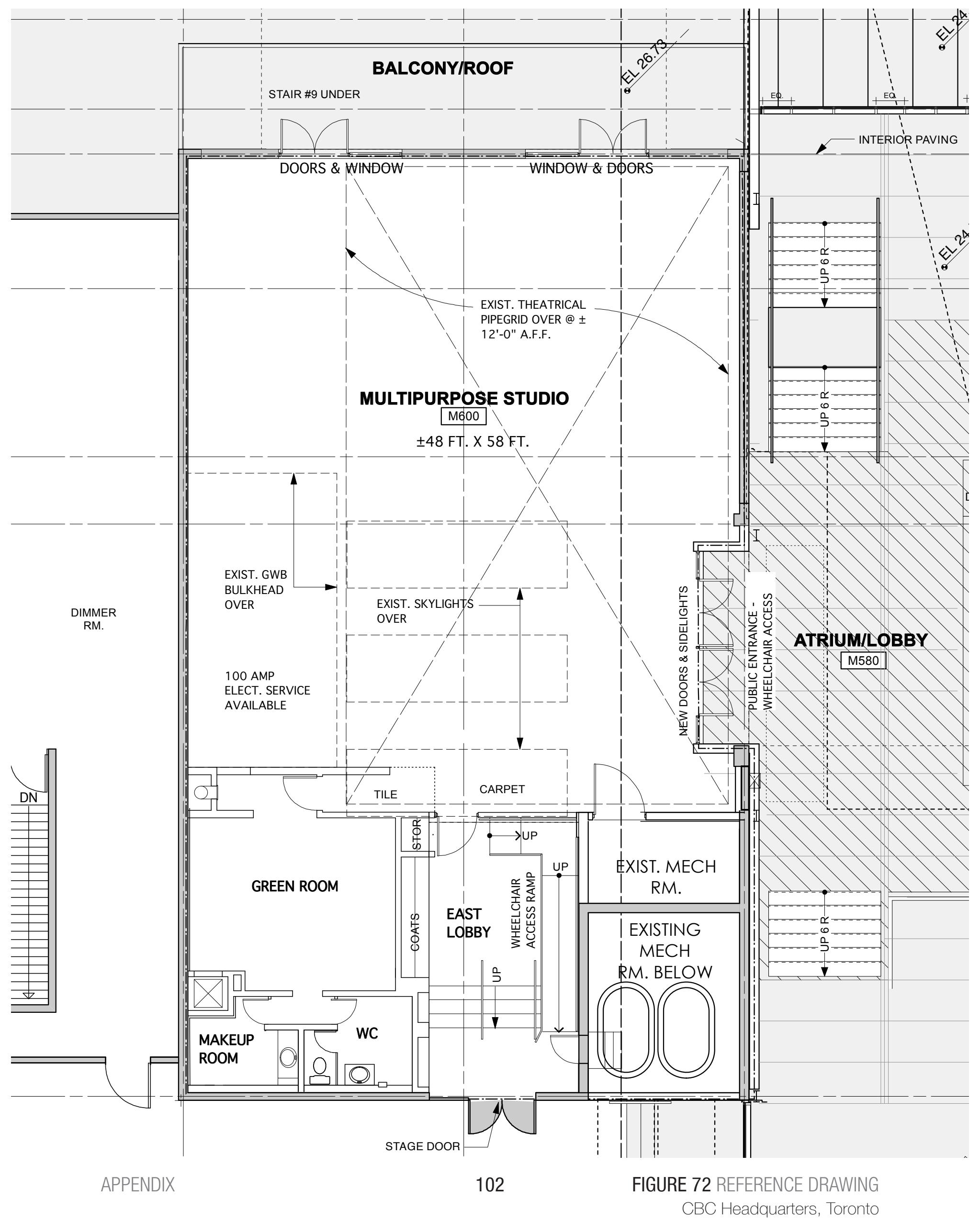




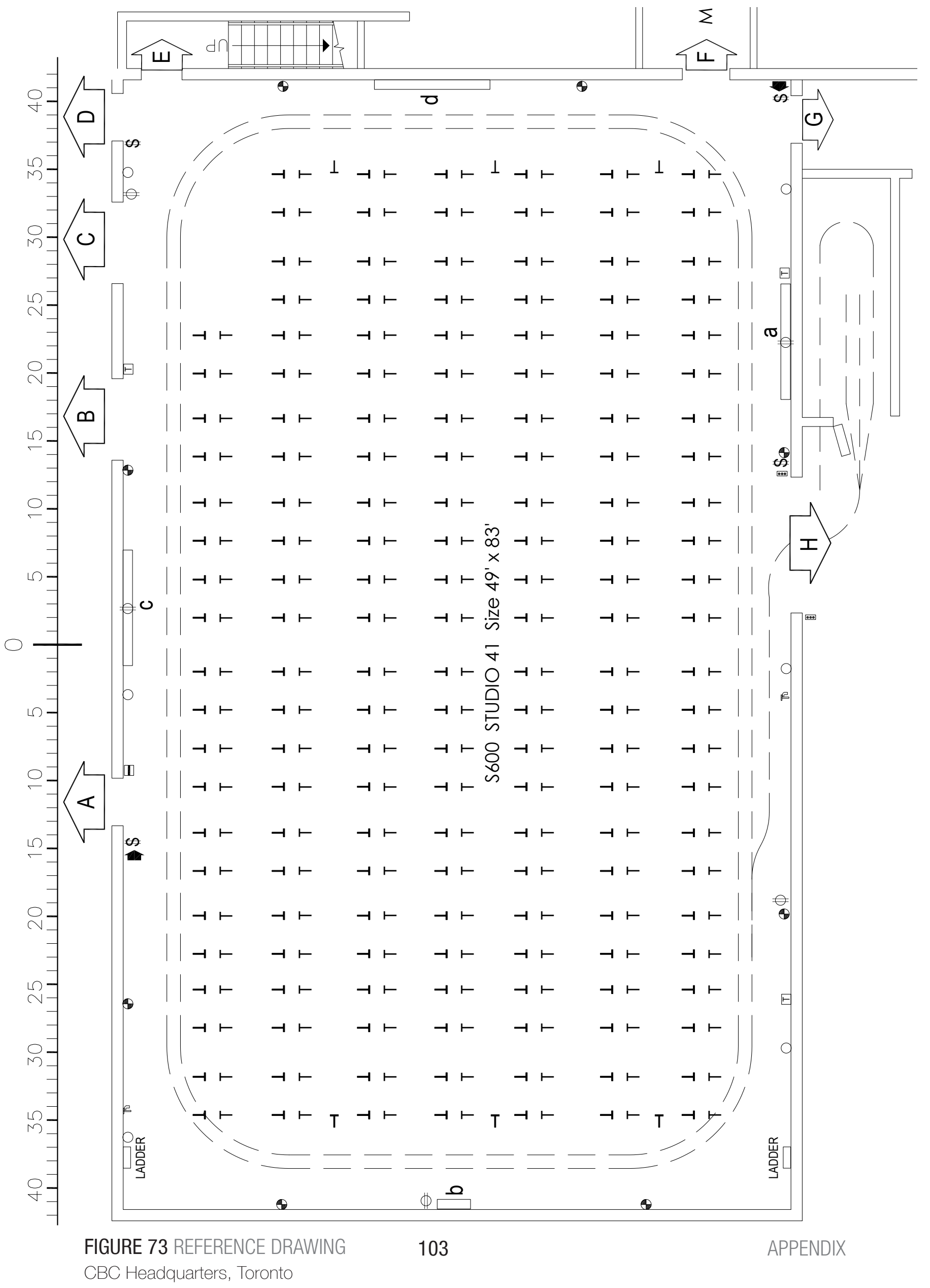




\section{BIBLIOGRAPHY}

Atwood, A. \& Niemark, A. (2015). Nine Essays. Chicago, Ml, USA: Treatise.

Baudrillard, J. (1994). Simulation and simulacra. (S. F. Glaser, Trans.) Ann Arbor, Michigan: University of Michigan Press.

Bayard, P. (2007). How to talk about books you haven't read. (J. Mehlman, Trans.) New York, USA: Bloomsberry.

Benjamin, W. (1936). The Work of art in the age of mechanical reproduction. Retrieved from http://www. marxists.org/references/subject/philosophy/works/ge/benjamin.htm

Berger, J. (1973). Ways of seeing. New York,NY: The Viking Press

Berkel, B. v., \& Bos, C. (2006). UN Studio: Design models - architecture, urbanism, infrastructure. Thames \& Hudson.

Calvino, I. (1988). Six memos for the next millenium. Cambridge MA: Harvard University Press.

Cohen, P. (2013) Successive Architecture [Lecture]. Cambridge: Harvard Graduate School of Design.

Colomina, B. (1987). Le Corbusier and photography. Assemblage, October 1987, 6-23

Croset, P. (1988). The Narration of architecture. In Joan Oakham (ed) Revisions 2: Architectureproduction (pp. 200-212). New York, NY: Princeton Architectural Press. 
Chua, Y. (2008 December - 2009 January). Promoting Harmony. Mark, 17, 126-137.

Eco, U. (1986). Travels in hyper reality: essays. San Diego, California: Harcourt Brace Jovanovich

Hays, K. (2010). Architecture's desire: reading of the late avant-garde. Cambridge, MA: MIT Press

Keats, J. (2012). Forged: Why Fakes Are the Great Art of Our Age. New York: Oxford University Press.

Kipnis, J. (2013). A question of qualities: Essays in architecture. Cambridge, Massachusetts: The MIT Press.

Kipnis, J. (2001) Perfect acts of architecture. New York, New York: Museum of Moddern Art.

Koepnick, L., \& McGlothlin, E. (2009). After the digital divide?: German aesthetic theory in the age of new media. New York: Camden House.

Manovich, L. (2005). Remixability and modularity. Retrieved from: http://manovich.net/index.php/projects/ remixability-and-modularity

Manovich, L. (2006). The Language of new media. Cambridge, Massachusetts: The MIT Press.

Manovich, L. (2007). What comes after remix?. Retrieved from: http://manovich.net/index.php/projects/ what-comes-after-remix

Mitchell, W. J. (2005). Placing words: Symbols, space, and the city. Cambridge: MIT Press.

Navas, E. (2013). Modular complexity and remix: The collapse of time and space into search. Retrieved from: http://remixtheory.net/wp-content/uploads/2013/03/NavasModFinalSmall121.pdf 
Navas, E. (2014) The Routledge companion to remix studies. New York, New York. Routledge.

Rodowick, D. N. (2007). The Virtual life of film. Cambridge: Harvard University Press.

Stott, R. (2014, February 24). Arch Daily. Retrieved from A Case for the Democratization of Architectural Media: http://umw.archdaily.com/480210/a-case-for-the-democratization-of-architectural-media/

Walker, E. (2006) Tschumi on architecture: conversations with enrique walker. New York, New York: Monacelli Press 